\title{
KANSAS AND OKLAHOMA.
}

\author{
PRIMARY TRAVERSE.
}

Columbus, Joplin, Parsons, and Wyandotte quadrangles.

CHEROKEE COUNTY.

In the spring of $1916 \mathrm{~J} . \mathrm{H}$. Wilson extended control for the abovenamed quadrangles from primary traverse stations previously located in near-by parts of Kansas and Missouri. (See Missouri list also.) The line was tied to Edwards triangulation station.

Positions are on approximate North American datum.

COLUMBUS QUADRANGLE, RANS.

South along highways near west border of quadrangle, north half. (By J. H. Wilson in 1916.)

\begin{tabular}{|c|c|c|c|}
\hline Station. & Latitude. & Longitude. & $\begin{array}{l}\text { Distance } \\
\text { between } \\
\text { stations. }\end{array}$ \\
\hline 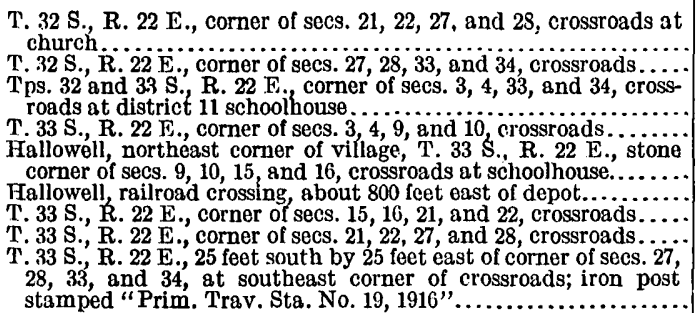 & $\begin{array}{lcc}0 & 1 & 11 \\
37 & 14 & 16.2 \\
37 & 13 & 23.5 \\
& & \\
37 & 12 & 30.8 \\
37 & 11 & 37.8 \\
37 & 10 & 45.1 \\
37 & 10 & 29.8 \\
37 & 09 & 52.3 \\
37 & 08 & 59.0 \\
37 & 08 & 05.7\end{array}$ & $\begin{array}{ccc}\circ & \prime & \prime \prime \\
94 & 59 & 42.2 \\
94 & 59 & 42.7 \\
& & \\
94 & 59 & 42.6 \\
94 & 59 & 42.8 \\
& & \\
94 & 59 & 42.2 \\
94 & 59 & 42.3 \\
94 & 59 & 42.5 \\
94 & 59 & 42.6\end{array}$ & $\begin{array}{r}\text { Feet. } \\
5,330 \\
5,335 \\
5,355 \\
5,330 \\
1,555 \\
3,785 \\
5,395 \\
\\
5,382\end{array}$ \\
\hline
\end{tabular}

Along highways through center of quadrangle.

T. 33 S., R. 22 E., corner of secs. $26,27,34$, and 35 , crossroads.... T. 33 S., R. 22 E., corner of secs. $25,26,35$, and 36 , crossroads..... T. 33 S., Rs. 22 and 23 E., corner of secs. 25,30 , 31, and 36, crossT. 33 S., R. 23 E., corner of secs. $29,30,31$, and 32 , crossroads......

T. 33 S., R. 23 E., corner of secs. $28,29,32$, and 33 , crossroads.

T. 33 S., R. $23 \mathrm{E}$., 25 feet south by 35 feet east of corner of secs. 27 , $2 S, 33$, and 34 , at southeast corner of crossroads; iron post stamped "Prim. Trav. Sta. No. 20, 1916".

\begin{tabular}{llll|rrr|r}
37 & 08 & 05.8 & 94 & 58 & 36.9 & $\ldots \ldots \ldots$, \\
37 & 08 & 05.7 & 94 & 57 & 31.5 & 5,300 \\
37 & 08 & 05.5 & 94 & 56 & 25.9 & \\
37 & 08 & 05.0 & 94 & 55 & 20.6 & \\
37 & 08 & 04.7 & 94 & 54 & 15.3 & & 5,285 \\
& & & & & \\
37 & 08 & 03.8 & 94 & 53 & 09.1 & & \\
& & & & &
\end{tabular}

East along hlghways through center of quadrangle.

\begin{tabular}{|c|c|c|c|}
\hline 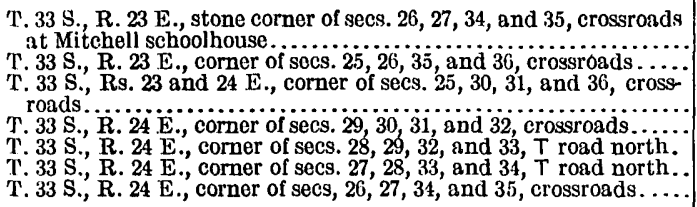 & $\begin{array}{lll}37 & 08 & 03.8 \\
37 & 08 & 03.5 \\
37 & 08 & 02.9 \\
37 & 08 & 02.7 \\
37 & 08 & 02.6 \\
37 & 08 & 02.6 \\
37 & 08 & 02.5\end{array}$ & $\begin{array}{lll}94 & 52 & 04.1 \\
94 & 50 & 58.7 \\
& & \\
94 & 49 & 53.4 \\
94 & 48 & 48.4 \\
94 & 47 & 42.8 \\
94 & 46 & 37.0 \\
94 & 45 & 31.4\end{array}$ & $\begin{array}{l}5,268 \\
5,292 \\
5,290 \\
5,260 \\
5,314 \\
5,303 \\
5,310\end{array}$ \\
\hline
\end{tabular}


COLUMBUS QUTARANGLE, KANS.-Continued.

West along St. Louis-San Francisco Ry. (1900 traverse line.)

\begin{tabular}{|c|c|c|c|}
\hline Station. & Latitude. & Longitude. & $\begin{array}{l}\text { Distance } \\
\text { between } \\
\text { stations. }\end{array}$ \\
\hline $\begin{array}{l}\text { T. } 33 \mathrm{~S} ., \mathrm{R} .24 \mathrm{~F} \text {, quarter corner between secs. } 16 \text { and } 17 \text {; rail- } \\
\text { road crossing is } 40 \text { leet south... } \\
\text { Columbus, east of, at crossing of Kansas Ċity. Pittsburg \& Gulf }\end{array}$ & $\begin{array}{ccc}\circ & \prime & \prime \prime \\
37 & 10 & 14.9\end{array}$ & $\begin{array}{ccc}\circ & \prime & \prime \prime \\
94 & 47 & 43.3\end{array}$ & $\begin{array}{l}\text { Feet. } \\
\ldots . . .\end{array}$ \\
\hline 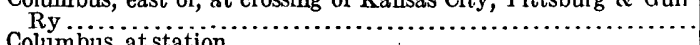 & $\begin{array}{lll}37 & 10 & 32.0\end{array}$ & $\begin{array}{lll}94 & 50 & 14.3\end{array}$ & 12,351 \\
\hline $\begin{array}{l}\text { Colúmbus, at station } \ldots \ldots \ldots \ldots \ldots \ldots \ldots \ldots \\
\text { Road crossing (see Wilson's traverse above) }\end{array}$ & 371030.8 & 945034.6 & 1,649 \\
\hline $\begin{array}{l}\text { Road crossing (see Wilson's traverse above) } \ldots \ldots \ldots \ldots \ldots \ldots \ldots \\
\text { Sherwin crossing of Missouri Pacific R R }\end{array}$ & 371043.8 & $94 \quad 54 \quad 15.2$ & 17,903 \\
\hline $\begin{array}{l}\text { Sherwin crossing of Missouri Pacific R. R... } \\
\text { T. } 33 \text { S., R.22 E., corner of secs. } 11,12,13 \text {, and } 14 ; \text { railioad crossing }\end{array}$ & 371045.1 & 945653.9 & 12,796 \\
\hline 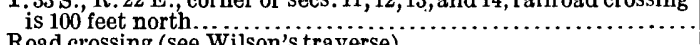 & $\begin{array}{llll}37 & 10 & 44.3\end{array}$ & 945731.4 & 3,041 \\
\hline 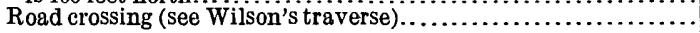 & $\begin{array}{llll}37 & 10 & 29.8\end{array}$ & 945942.3 & 2,450 \\
\hline
\end{tabular}

Magnetic declination for north and south borders and along center line of quadrangle, $8.8^{\circ} \mathrm{E}$; for west border of quadrangle, $8.9^{\circ} \mathrm{E}$.

South along highways along south half of west border of quadrangle.

Tps. 33 and 34 S., R. 22 E., corner of secs. 3, 4, 33, and 34, crossroads T. 34 S., R. 22 E., corner of secs. $3,4,9$, and 10, 1 mile east of Faulk-

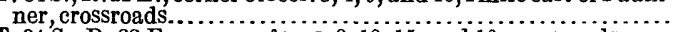

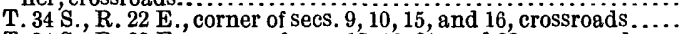
T. 34 S., R. 22 E., corner of secs. 15, 16, 21, and 22 , crossroads... T. 34 S., R. 22 E., corner of secs. $21,22,27$, and 28 , crossroads.. Pleasant Valley schoolhouse, T. 34 S., R. 22 E., corner of secs. 27 , 28,33 , and 34 , crossroads...

Tps. 34 and 35 S., R. 22 E., corner of secs. $3,4,33$, and 34 , crossroads Fairview schoolhouse, T. 35 S., R. 22 E., corner of secs. $3,4,9$, and 10 , crossroads

T. 35 S., R. 22 E., corner of sees. $9,10,15$, and $16,{ }^{\prime}$ T road east and

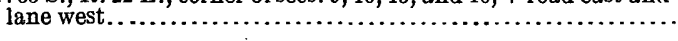

\begin{tabular}{|c|c|c|}
\hline $\begin{array}{llll}37 & 07 & 13.1\end{array}$ & 945942.6 & \\
\hline $\begin{array}{lll}37 & 06 & 18.7 \\
37 & 05 & 24.6 \\
37 & 04 & 32.2 \\
37 & 03 & 41.0\end{array}$ & $\begin{array}{lll}94 & 59 & 42.6 \\
94 & 59 & 42.7 \\
94 & 59 & 42.4 \\
94 & 59 & 41.8\end{array}$ & $\begin{array}{l}5,500 \\
5,460 \\
5,295 \\
5,175\end{array}$ \\
\hline $\begin{array}{lll}37 & 02 & 48.5 \\
37 & 01 & 55.6\end{array}$ & $\begin{array}{lll}94 & 59 & 41.5 \\
94 & 59 & 41.5\end{array}$ & $\begin{array}{l}5,305 \\
5,345\end{array}$ \\
\hline $\begin{array}{lll}37 & 01 & 03.0\end{array}$ & 945941.5 & 5,320 \\
\hline $\begin{array}{lll}37 & 00 & 10.6\end{array}$ & $\begin{array}{lll}94 & 59 & 41.7\end{array}$ & 5,300 \\
\hline
\end{tabular}

\section{At Edwards triangulation station.}

\begin{tabular}{|c|c|c|}
\hline 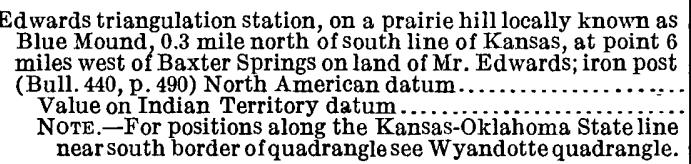 & $\begin{array}{lll}37 & 00 & 13.2 \\
37 & 00 & 14.6\end{array}$ & $\begin{array}{lll}94 & 49 & 25.9 \\
94 & 49 & 23.9\end{array}$ \\
\hline
\end{tabular}

JOPLIN 30' QUADRANGLE, NORTHEAST QUARTER, KANS.

Near southwest corner of $1^{\prime}$ quadrangle. (By J. H. Wilson in 1916.)

\begin{tabular}{|c|c|c|c|}
\hline Station. & Latitude. & Longitude. & $\begin{array}{l}\text { Distance } \\
\text { between } \\
\text { stations. }\end{array}$ \\
\hline $\begin{array}{l}\text { T. } 32 \text { S., R. } 24 \text { E., } 20 \text { feet south by } 30 \text { feet west of corner secs. } 13,14, \\
23 \text {, and } 24 \text {, at southwest corner of crossroads; iron post stamped } \\
\text { "Prim. Trav. Sta. No. } 16,1916 " \ldots \ldots \ldots \ldots \ldots \ldots \ldots \ldots \ldots \ldots\end{array}$ & 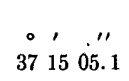 & $\begin{array}{ccc}\circ & \prime & \prime \prime \\
94 & 44 & 28.8\end{array}$ & $\begin{array}{c}\text { Feet. } \\
\ldots . . .\end{array}$ \\
\hline
\end{tabular}

Magnetic declination for southwest corner of quadrangle, $8.7^{\circ} \mathrm{E}$. 
JOPIIN 30' QUADRANGLE, NORTHWEST QUARTER, KANS.

West along highways near south border of quadrangle. (By J. H. Wilson in 1916.)

\begin{tabular}{|c|c|c|c|}
\hline Station. & Latitude. & Longitude. & $\begin{array}{l}\text { Distance } \\
\text { between } \\
\text { stations. }\end{array}$ \\
\hline 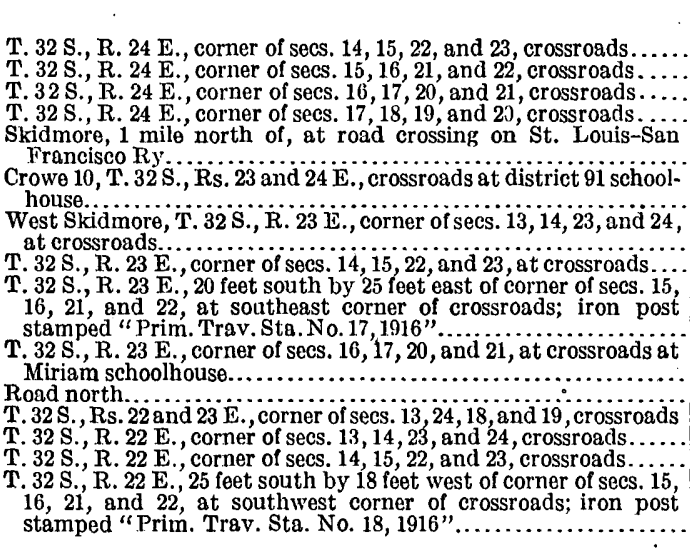 & $\begin{array}{lll}37 & 15 & 07.4 \\
37 & 15 & 07.6 \\
& & \\
37 & 15 & 07.5 \\
& & \\
37 & 15 & 08.1 \\
37 & 15 & 08.6 \\
37 & 15 & 09.3 \\
37 & 15 & 08.7 \\
37 & 15 & 08.4\end{array}$ & $\begin{array}{lll}94 & 50 & 59.9 \\
94 & 52 & 05.3 \\
& & \\
94 & 53 & 10.5 \\
& & \\
94 & 54 & 16.4 \\
94 & 55 & 18.9 \\
94 & 56 & 25.3 \\
94 & 57 & 30.6 \\
94 & 58 & 35.8\end{array}$ & $\begin{array}{r}\text { Feet. } \\
\ldots, 260 \\
5,310 \\
5,270 \\
. \\
3,600 \\
1,625 \\
5,295 \\
5,290\end{array}$ \\
\hline
\end{tabular}

Magnetic declination for south border of quadrangle, $8.8^{\circ} \mathrm{E}$.

\section{JOPLIN DISTRICT QUADRANGLE, KANS.}

West along highways near State line and north border of quadrangle. (By J. H. Wilson in 1916.)

\begin{tabular}{|c|c|c|c|}
\hline Station. & Latitude. & Longitude. & $\begin{array}{l}\text { Distance } \\
\text { between } \\
\text { stations. }\end{array}$ \\
\hline 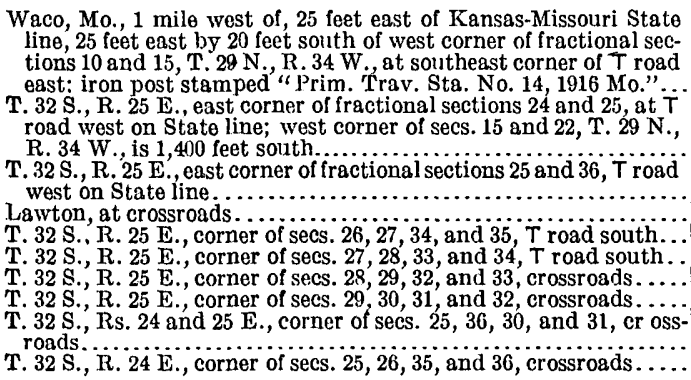 & $\begin{array}{ccc}0 & \prime & \prime \prime \\
37 & 14 & 50.4 \\
- & & \\
37 & 14 & 12.3 \\
37 & 13 & 19.9 \\
37 & 13 & 20.0 \\
37 & 13 & 20.5 \\
37 & 13 & 20.2 \\
37 & 13 & 20.0 \\
37 & 13 & 19.8 \\
& & \\
37 & 13 & 19.6 \\
37 & 13 & 19.9 .\end{array}$ & $\begin{array}{ccc}\circ & \prime & \prime \prime \\
94 & 37 & 03.8 \\
& & \\
94 & 37 & 04.3 \\
94 & 37 & 04.4 \\
94 & 38 & 09.5 \\
94 & 38 & 58.9 \\
94 & 40 & 05.0 \\
94 & 41 & 11.3 \\
94 & 42 & 16.8\end{array}$ & $\begin{array}{r}3,850 \\
5,300 \\
5,270 \\
3,994 \\
5,345 \\
5,360 \\
5,300\end{array}$ \\
\hline
\end{tabular}

South along highways near west border of quadrangle.

T. 32 S., R. 24 E., corner of secs. $23,24,25$, and 26 , just east of steel bridge over Shawnee Creek, crossroads

Tps. 32 and 33 S., R. 24 E., corner of secs. $1,2,35$, and 36 , crossroads. T. 33 S., R. 24 E., corner of secs. 1, 2, 11, and 12, crossroads.

T. $33 \mathrm{~S}, \mathrm{R}, 24 \mathrm{E}$ corner of secs $11,12,13$ and 14, crossroads

T. 33 S., R. 24 E., quarter corner between secs. 13 and 14, railroad crossing.

\begin{tabular}{lll|lll|l}
37 & 14 & 12.6 & 94 & 44 & 27.9 & \\
37 & 12 & 26.9 & 94 & 44 & 26.9 & 5,328 \\
37 & 11 & 34.2 & 94 & 44 & 26.7 & 5,362 \\
37 & 10 & 41.2 & 94 & 44 & 26.5 & 5,327 \\
37 & 10 & 14.4 & 94 & 44 & 26.5 & \\
& & & & &
\end{tabular}


JOPLIN DISTRICT QUADRANGLE, KANS.-Continued.

(Hawkins 1900 traverse.)

\begin{tabular}{|c|c|c|c|}
\hline Station. & Latitude. & Longitude. & $\begin{array}{l}\text { Distance } \\
\text { between } \\
\text { stations. }\end{array}$ \\
\hline 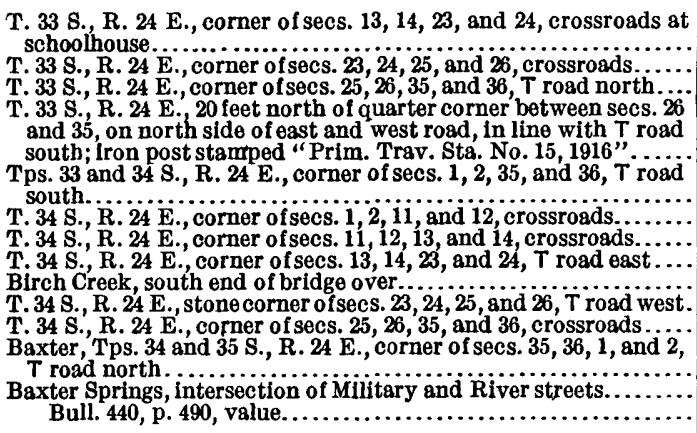 & $\begin{array}{lll} & \prime & \prime \prime \\
37 & 09 & 48.1 \\
37 & 08 & 55.3 \\
37 & 08 & 02.4 \\
& & \\
37 & 08 & 02.8 \\
& & \\
37 & 07 & 09.4 \\
37 & 06 & 17.1 \\
37 & 05 & 24.7 \\
37 & 04 & 32.3 \\
37 & 04 & 24.3 \\
37 & 03 & 39.5 \\
37 & 02 & 45.6 \\
& & \\
37 & 01 & 52.1 \\
37 & 01 & 25.2 \\
37 & 01 & 26.6\end{array}$ & $\begin{array}{ccc}\circ & \prime & \prime \prime \\
94 & 44 & 26.4 \\
94 & 44 & 25.9 \\
94 & 44 & 25.9 \\
& & \\
94 & & \\
94 & 58.4 \\
94 & & \\
94 & 25.7 \\
94 & 44 & 25.9 \\
94 & 44 & 26.2 \\
94 & 44 & 26.4 \\
94 & 44 & 26.8 \\
94 & 44 & 26.2 \\
94 & 44 & 25.7\end{array}$ & $\begin{array}{r}\text { Feet. } \\
2,655 \\
5,335 \\
5,344 \\
\\
2,637\end{array}$ \\
\hline
\end{tabular}

Magnetic declination for north border of quadrangle, $8.4^{\circ} \mathrm{E}$; for west border of quadrangle, $8.7^{\circ} \mathrm{E}$.

Northwest along St. Louis-San Francisco Ry. (B!r G. T. Hawkins in 1900.)

\begin{tabular}{|c|c|c|c|}
\hline 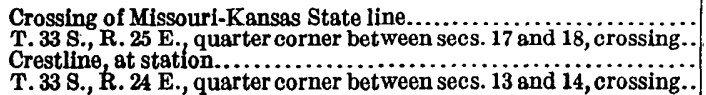 & $\begin{array}{lll}37 & 09 & 58.4 \\
37 & 10 & 14.2 \\
37 & 10 & 14.2 \\
37 & 10 & 14.4\end{array}$ & $\begin{array}{lll}94 & 37 & 04.4 \\
94 & 42 & 15.0 \\
94 & 42 & 18.9 \\
94 & 44 & 26.5\end{array}$ & $\begin{array}{r}25,194 \\
310 \\
10,320\end{array}$ \\
\hline
\end{tabular}

PARSONS QUADRANGLE, BANS.

Along St. Louis-San Franclsco Ry. (a spur line of 1900 to Oswego Astronomical Station.) (By J. H. Wilson in 1916.)

\begin{tabular}{|c|c|c|c|}
\hline Station. & Latitude. & Longitude. & $\begin{array}{l}\text { Distance } \\
\text { between } \\
\text { stations. }\end{array}$ \\
\hline 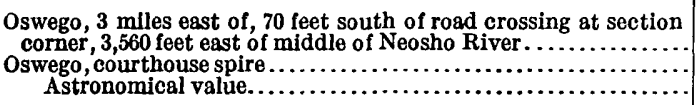 & \begin{tabular}{ccc|}
$\circ$ & $\prime$ & $\prime \prime$ \\
37 & 09 & 52.7 \\
37 & 09 & 55.3 \\
37 & 09 & 58.9
\end{tabular} & $\begin{array}{ccc}\circ & \prime \prime \prime \\
95 & 02 & 59.2 \\
95 & 06 & 26.9 \\
95 & 06 & 24.1\end{array}$ & $\begin{array}{l}\text { Feet. } \\
16,813 \\
\end{array}$ \\
\hline
\end{tabular}


WYANDOTTE QUADRANGLE, KANS.-OKLA.

East along highways near State line (north border of quadrangle). (By J, H. Wilson in 1916.)

\begin{tabular}{|c|c|c|c|}
\hline Station. & Latitude. & Longitude. & $\begin{array}{l}\text { Distance } \\
\text { between } \\
\text { stations. }\end{array}$ \\
\hline $\begin{array}{l}\text { T. } 35 \mathrm{~S}, \text {, R. } 22 \mathrm{E} \text {, } 20 \text { feet south of south corner of secs. } 15 \text { and } 16 \text { and } \\
\text { Kansas Oklahoma State line, on south side of road, opposite T } \\
\text { road north; iron post stamped "Prim. Trav. Sta. No. 21,1916" } \\
\text { NoTE.-T road south at north corner of secs. } 13 \text { and 14, T. } 29 \\
\text { N. R. } 21 \mathrm{E} \text {. is } 15 \text { feet north by } 630 \text { feet east. }\end{array}$ & 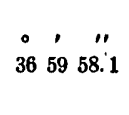 & 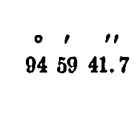 & Feet. \\
\hline 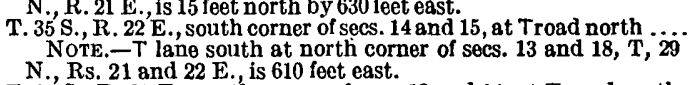 & $\begin{array}{lll}36 & 59 & 58.1\end{array}$ & 945836.3 & 5,300 \\
\hline 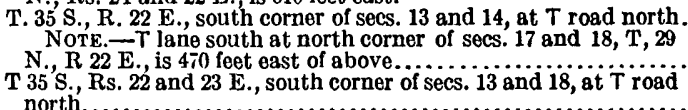 & $\begin{array}{lll}36 & 59 & 57.9\end{array}$ & 945730.6 & 5,330 \\
\hline $\begin{array}{l}\text { T. } 35 \mathrm{~S} ., \mathrm{R} .23 \mathrm{E} ., \text { south corner of secs. } 17 \text { and } 18 \text { at } \mathrm{T} \text { road north..... } \\
\text { of road. - From above corner eastward the State line is in center }\end{array}$ & $\begin{array}{lll}36 & 59 & 57.3 \\
36 & 59 & 57.0\end{array}$ & $\begin{array}{lll}94 & 56 & 25.3 \\
94 & 55 & 20.0\end{array}$ & $\begin{array}{l}5.300 \\
5,295\end{array}$ \\
\hline $\begin{array}{l}\text { T. } 35 \text { S., R. } 23 \mathrm{E} \text {, south corner of secs. } 16 \text { and } 17, \mathrm{~T} \text { road north..... } \\
\text { Nore.- North corner of secs. } 14 \text { and } 15, \mathrm{~T} .29 \text { N., R. } 22 \mathrm{E} \text {., is } 410 \\
\text { feet east of above. }\end{array}$ & 365956.5 & 945414.6 & 5,300 \\
\hline $\begin{array}{l}\text { T. } 35 \text { S., R. } 23 \text { E., south corner of secs. } 15 \text { and } 16, T \text { road north...... } \\
\text { NoTE. North corner of secs. } 13 \text { and 14, T. } 29 \text { N., R.22 E., at } T \\
\text { road south is } 420 \text { feet east. }\end{array}$ & $\begin{array}{lll}36 & 59 & 56.0\end{array}$ & 945309.4 & 5,290 \\
\hline $\begin{array}{l}\text { T. } 35 \text { S., R. } 23 \text { E., south corner of secs. } 14 \text { and } 15, T \text { road north....... } \\
\text { Note.-North corner of secs. } 13 \text { and } 18, T \text {. } 29 \text { N., Rs. } 22 \text { and } 23 \\
\text { E., at road south is } 300 \text { feet east. }\end{array}$ & $\begin{array}{lll}36 & 59 & 56.2\end{array}$ & 945203.5 & 5,350 \\
\hline $\begin{array}{l}\text { T. } 35 \text { S., R. } 23 \text { E., south corner of secs. } 13 \text { and } 14, T \text { road north...... } \\
\text { T. } 35 \text { S., Rs. } 23 \text { and } 24 \text { E., south corner of secs. } 23 \text { and } 18, T \text { road }\end{array}$ & $\begin{array}{lll}36 & 59 & 56.0\end{array}$ & 945058.1 & 5,299 \\
\hline $\begin{array}{l}\text { north.... North corner of secs. } 16 \text { and } 17, \mathrm{~T} .29 \mathrm{~N} ., \mathrm{R} .24 \mathrm{E} \text {., at } \mathrm{T} \\
\text { road south is } 160 \text { feet east. }\end{array}$ & $\begin{array}{l}365955.8 \\
\end{array}$ & 944952.5 & 5,320 \\
\hline
\end{tabular}

Along highways near State line (north border of quadrangle) by traverse of 1906, taken from Bulletin 440, page 490, corrected.

\begin{tabular}{|c|c|c|c|}
\hline 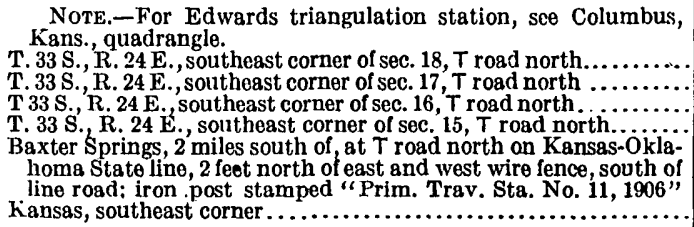 & $\begin{array}{lll}36 & 59 & 55.6 \\
36 & 59 & 55.8 \\
36 & 59 & 55.4 \\
36 & 59 & 55.0\end{array}$ & $\begin{array}{lll}94 & 48 & 47.2 \\
94 & \mathbf{4 7} & \mathbf{4 1 . 5} \\
94 & \mathbf{4 6} & 36.1 \\
94 & \mathbf{4 5} & 30.5\end{array}$ & $\begin{array}{r}5,330 \\
5,305 \\
5,322\end{array}$ \\
\hline
\end{tabular}

Magnetic declination for north border, $1916,8.7^{\circ} \mathrm{E}$.

\section{MISSOURI.}

PRIMARY TRAVERSE.

Carthage, Greenfield, Halltown, Joplin, Sarcoxie, Springfield, and Stotts City quadrangles.

DADE, JASPER, AND LAWRENCE COUNTIES.

In the spring of $1916 \mathrm{~J}$. H. Wilson extended control for the abovenamed quadrangles from primary traverse stations previously located in this vicinity. The positions are on approximate North American datum.

For other positions in Missouri see U. S. Geological Survey Bulletins 122, 181, 201, 216, 245, 310, 440, 496, 551, 644, Nineteenth Annual Report, PartI; U. S. Coast and Geodetic Survey Special Publication No. 
30, Missouri River Commission Reports for 1891 and 1893, Mississippi River Commission Reports for 1883, 1888, and 1891, also Appendix 22, House Document 50, Sixty-first Congress.

CARTHAGE 30' QUADRANGLE.

West along highways through center of quadrangle. (By J. H. Wilson in 1916.)

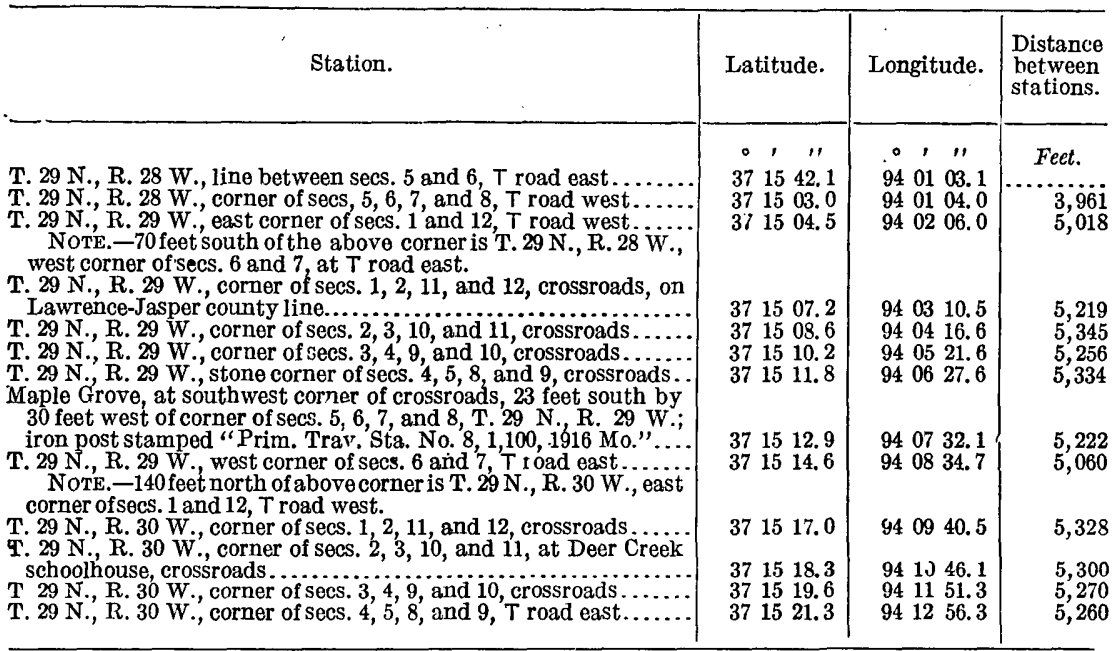

Magnetic declination for south border of quadrangle, $7.7^{\circ} \mathrm{E}$.

GREENFIELD QUADRANGLE, NORTHEAST QUARTER.

Northwest along St. Louls-San Francisco Ry. (1900 traverse line). (By J. H. Wilson in 1916.)

\begin{tabular}{|c|c|c|c|}
\hline Station. & Latitude. & Longitude. & $\begin{array}{l}\text { Distance } \\
\text { between } \\
\text { stations. }\end{array}$ \\
\hline 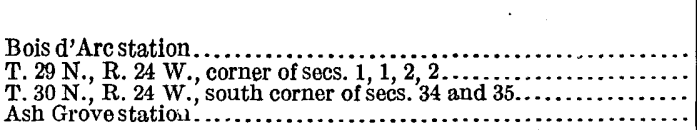 & $\begin{array}{lll}\circ & , & \prime \prime \\
37 & 15 & 27.3 \\
37 & 15 & 30.5 \\
37 & 16 & 49.9 \\
37 & 18 & 59.0\end{array}$ & $\begin{array}{ccc}\circ & \prime & \prime \prime \\
93 & 30 & 24.0 \\
93 & 30 & 44.5 \\
93 & 33 & 08.1 \\
93 & 35 & 12.3\end{array}$ & $\begin{array}{l}\text { Feet. } \\
\cdots \cdots \cdots \\
\cdots \cdots \\
\cdots \cdots \\
\cdots \cdots\end{array}$ \\
\hline
\end{tabular}

West along highway in southeast quarter of quadrangle.

T. 29 N., R. 24 W., corner of secs. $2,2,3,3$, center of east and west road at north and south fence line........................................... long north and south. Thisline of traverse seems to run through them about 1 mile from the south border.

T. 29 N., R. 24 W., corner of secs. 3, 3, 4, 4, T road south at Fair-

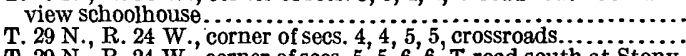
T. 29 N., R. 24 W., corner of secs. $5,5,6,6$, T road south at Stony

Point schoolhouse and church.

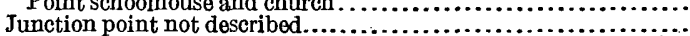

Tps. 29 and 30 N., Rs. 24 and 25 w., closing coiner at $T$ road south, northeast corner of Lawrence County, 21 feet northwest of iron pipe fence post with a concrete base...

T. 30 N., R. 24 W., south corner of secs. 32 and $33, T$ road west,

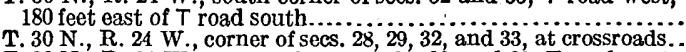
T. 30 N., R. 24 W., corner of secs. $20,21,28$, and 29 , T road west,

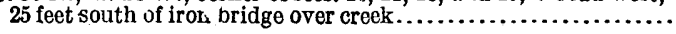

371530.5

371530.8 $\begin{array}{llll}37 & 15 & 31.1\end{array}$

$37 \quad 1530.9$ 371530.9

371653.8

371652.4 371744.8

371837.1
933150.1

$93 \quad 3255.7$ 933401.4

5,300

\begin{tabular}{lll|l}
93 & 35 & 07.6 & 5,345
\end{tabular}

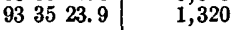

$93 \quad 3517.1$

9,417

933520.0

4,610

$93 \begin{array}{lll}35 & 18.3\end{array}$

5,294

933516.6 
GREENFIELD QUADRANGLE, NORTHEAST QUARTER-Continued.

West along highway near west half of south border of quadrangle.

\begin{tabular}{|c|c|c|c|}
\hline Station. & Latitude. & Longitude. & $\begin{array}{l}\text { Distance } \\
\text { between } \\
\text { stations. }\end{array}$ \\
\hline 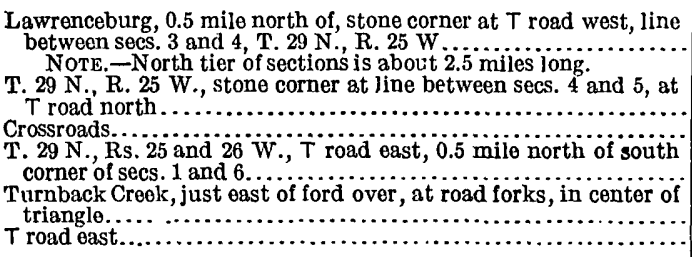 & $\begin{array}{lll}0 & \prime & \prime \prime \\
37 & 15 & 07.7 \\
37 & 15 & 08.4 \\
37 & 15 & 08.8 \\
37 & 15 & 10.2 \\
37 & 15 & 14.1 \\
37 & 15 & 01.9\end{array}$ & $\begin{array}{cccc}\bullet & \prime & \prime \prime \\
93 & 39 & 35.5 \\
& & & \\
93 & 40 & 40.7 \\
93 & 41 & 17.6 \\
93 & 42 & 39.7\end{array}$ & \begin{tabular}{|l} 
Feet. \\
\\
$\mathbf{5}, 272$ \\
$\mathbf{2}, 085$ \\
\\
6,636 \\
\\
$\mathbf{5}, 023$ \\
$\mathbf{3}, 219$
\end{tabular} \\
\hline
\end{tabular}

Magnetic declination for south border of quadrangle, $7.7^{\circ} \mathrm{E}$.

GREENFIELD QUADRANGLE, NORTHWEST QUARTER.

West along highways near south border of quadrangle. (By J. H. Wilson in 1916.)

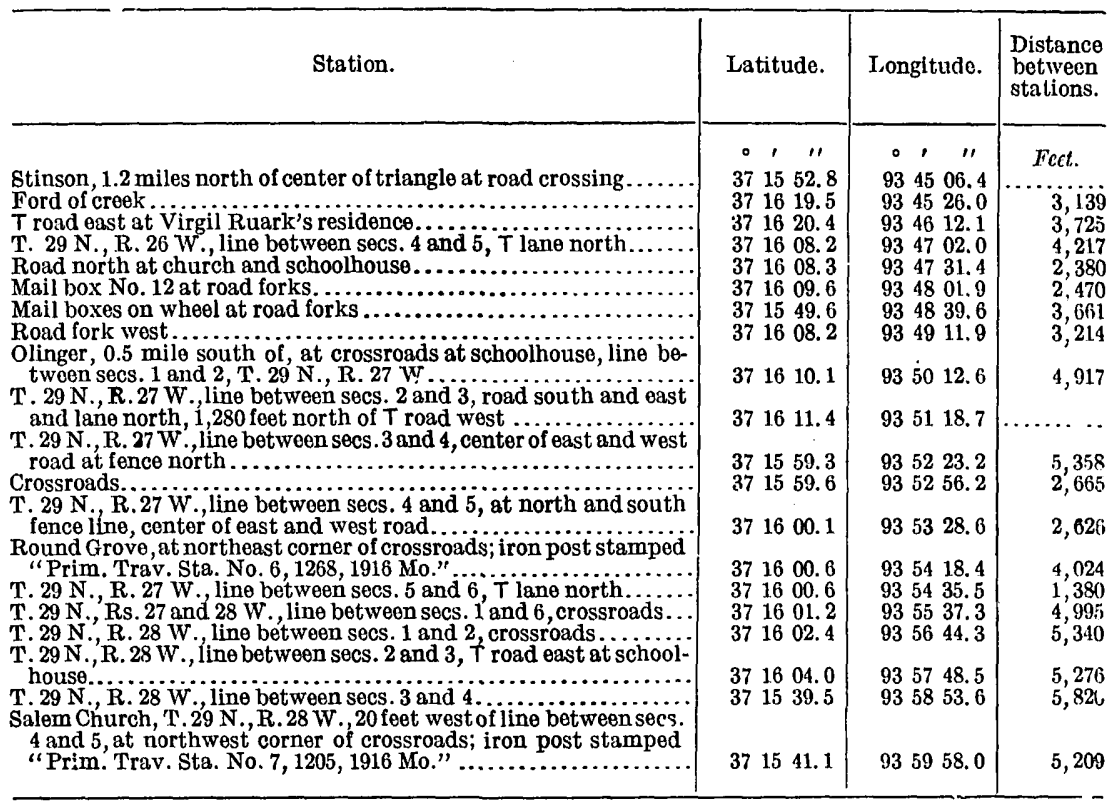

Magnetic declination for south border of quadrangle, $7.3^{\circ} \mathrm{E}$.

HALLTOWN QUADRANGLE.

South along St. Louis-Sun Francisco Ry. (By J. H. Wilson in 1916.)

\begin{tabular}{|c|c|c|c|}
\hline Station. & Latitudo. & Longiturde. & $\begin{array}{l}\text { Distance } \\
\text { between } \\
\text { stations. }\end{array}$ \\
\hline 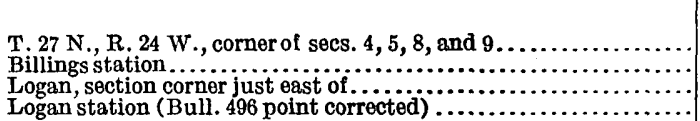 & $\begin{array}{ccc}\circ & , & \prime \prime \\
37 & 04 & 06.9 \\
37 & 04 & 00.2 \\
37 & 01 & 03.8 \\
37 & 00 & 48.6\end{array}$ & $\begin{array}{ccc}\circ & , & \prime \prime \\
93 & 33 & 14.9 \\
93 & 33 & 16.4 \\
93 & 36 & 34.5 \\
93 & 36 & 57.5\end{array}$ & \begin{tabular}{c}
\multicolumn{2}{c}{ Feet. } \\
$\cdots \cdots$ \\
$\cdots \cdots$ \\
$\cdots \cdots$ \\
$\cdots \cdots$ \\
$\cdots \cdots$
\end{tabular} \\
\hline
\end{tabular}


HALLTOWN QUADRATGLE-Continued.

Along highways near southeast border of quadrangle.

\begin{tabular}{|c|c|c|c|}
\hline Station. & Latitude. & Longitude. & $\begin{array}{l}\text { Distance } \\
\text { between } \\
\text { stations. }\end{array}$ \\
\hline 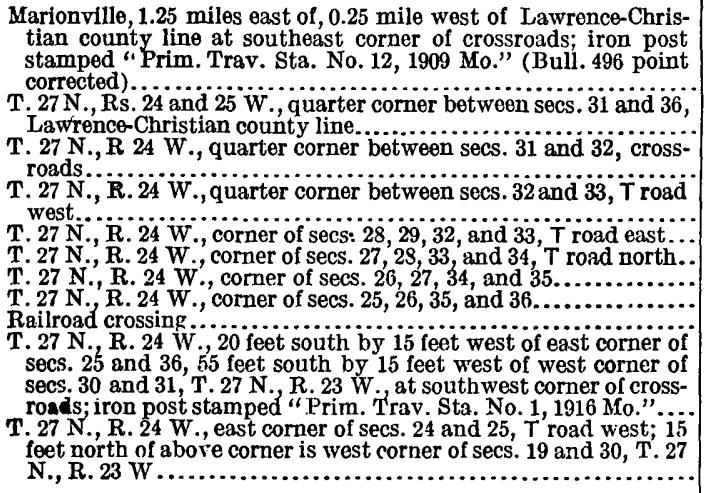 & \begin{tabular}{cccc}
$\circ$ & \multicolumn{1}{c}{ " } \\
37 & 00 & 11.3 \\
37 & 00 & 11.3 \\
& & \\
37 & 00 & 10.8 \\
& & & \\
37 & 00 & 10.0 \\
37 & 00 & 36.2 \\
37 & 00 & 35.3 \\
37 & 00 & 34.4 \\
37 & 00 & 33.0 \\
37 & 00 & 32.7 \\
& &
\end{tabular} & $\begin{array}{lll}93 & 34 & 25.1 \\
93 & 34 & 24.7 \\
93 & 33 & 19.2 \\
93 & 32 & 12.7 \\
93 & 31 & 07.3 \\
93 & 30 & 48.2\end{array}$ & $\begin{array}{l}\text { Feet. } \\
\ldots . . . \\
1,294 \\
5,190\end{array}$ \\
\hline
\end{tabular}

Southwest along highways in north center of quadrangle.

T. 29 N., R. 25 W., cast corner of secs. 1 and 12, at bend in road, on I.awrence-Greene county line, 0.25 mile east of crossroads....... T. 29 N., R. 25 W., corner of secs. 1, 2, 11, and 12, at crossroads; iron post stamped "Prim. Trav. Sta. No. 4, 1916 Mo.".

T. 29 N., R. 25 W., corner of secs. $11,12,13$, and 14 , crossroads.... T. 29 N., R. 25 W., corner of secs. $13,14,23$, and $24, T$ road east ...

T. 29 N., R. 25 W., corner of secs. $23,24,25$, and 26 , center of north

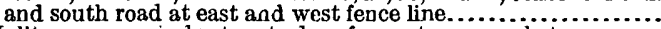
Halltown, crossroads at east edge of, quarter corner between secs. 25 and 26, T. 29 N., R. 25 W.

T. 29 N., R. 25 W., quarter-corner stone in center of sec. $26, T$

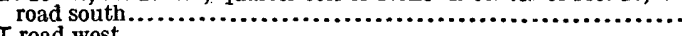

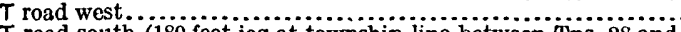

$T$ road south (180 feet jog at township line between $\mathrm{T}$ ps. 28 and 29 N.).

T. 28 N., $\mathrm{R} .25 \mathrm{~W}$. , quarter-corner stone between secs. 2 and $i 1$, at Roper schoolhouse crossroads.

T. 28 N., R. 25 W., stone quarter-corner between secs. 11 and 14 ,

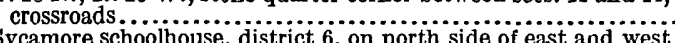
Sycamore schoolhouse, district 6 , on north side of east and west
road, opposite front door; iron post stamped "Prim. Truv. Sta. No. 1, 1909 Mo." (Bull. 496 point corrected)

\begin{tabular}{llll|lll|}
37 & 14 & 39.8 & 93 & 36 & 19.2 & $\ldots \ldots$ \\
37 & 14 & 39.0 & 93 & 37 & 24.7 & \\
37 & 13 & 46.9 & 93 & 37 & 25.9 & 5,295 \\
37 & 12 & 54.7 & 93 & 37 & 27.4 & 5,276 \\
37 & 12 & 02.5 & 93 & 37 & 28.6 & 5,285 \\
37 & 11 & 36.5 & 93 & 37 & 29.25 & 5,280 \\
37 & 11 & 37.2 & 93 & 38 & 02.1 & 2,631 \\
37 & 10 & 56.9 & 93 & 38 & 19.4 & 2,656 \\
37 & 10 & 19.1 & 93 & 38 & 22.3 & 4,313 \\
37 & 09 & 23.8 & 93 & 38 & 06.7 & 3,826 \\
37 & 08 & 31.5 & 93 & 38 & 07.3 & 5,744 \\
& & & & 5,289 \\
37 & 07 & 39.5 & 93 & 37 & 51.0 & 5,424 \\
\hline
\end{tabular}

West along highways near north border of quadrangle.

T. 29 N., R. 25 W., corner of secs. $2,3,10$, and 11, just west of Pilgrim's Rest Church, center of east and west road at fence line... Lawrenceburg, cormer of secs. 3, 4, 9, and 10, T. 29 N., R. 25 W., at

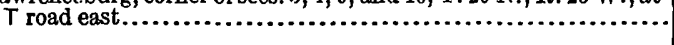

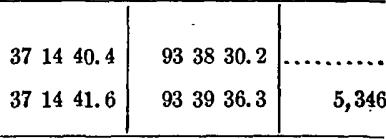

Near northwest corner of quadrangle.

\begin{tabular}{l|r|r|r}
\hline T. 29 N., R. 26 W., corner of secs. $2,3,10$, and 11, T lane south.... & 371447.3 & 934451.5 & $\ldots \ldots \ldots$ \\
\hline
\end{tabular}

Along highways near west border.

T. 28 N., R. 26 W., north corner of secs. 2 and 3 , at bend in road; 35 feet east of above corner is T. 29 N., R. 26 W., south corner of

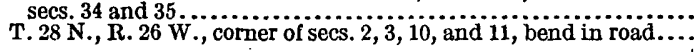

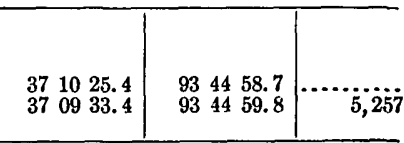

Magnetic declination (1916) for southeast corner of quadrangle, $7.2^{\circ} \mathrm{E}$; f for east and west borders of quadrangle, $7.5^{\circ} \mathrm{E}$.; for north border of quadrangle, $7.7^{\circ} \mathrm{E}$.; for north center of quadrangle, $7.8^{\circ} \mathrm{E}$. 
JOPLIN DISTRICT QUADRANGLE.

South along highways near east border of quadrangle. (By J. H. Wilson in 1916.)

\begin{tabular}{|c|c|c|c|}
\hline Station. & Latitude. & Longitude. & $\begin{array}{l}\text { Distance } \\
\text { between } \\
\text { stations. }\end{array}$ \\
\hline 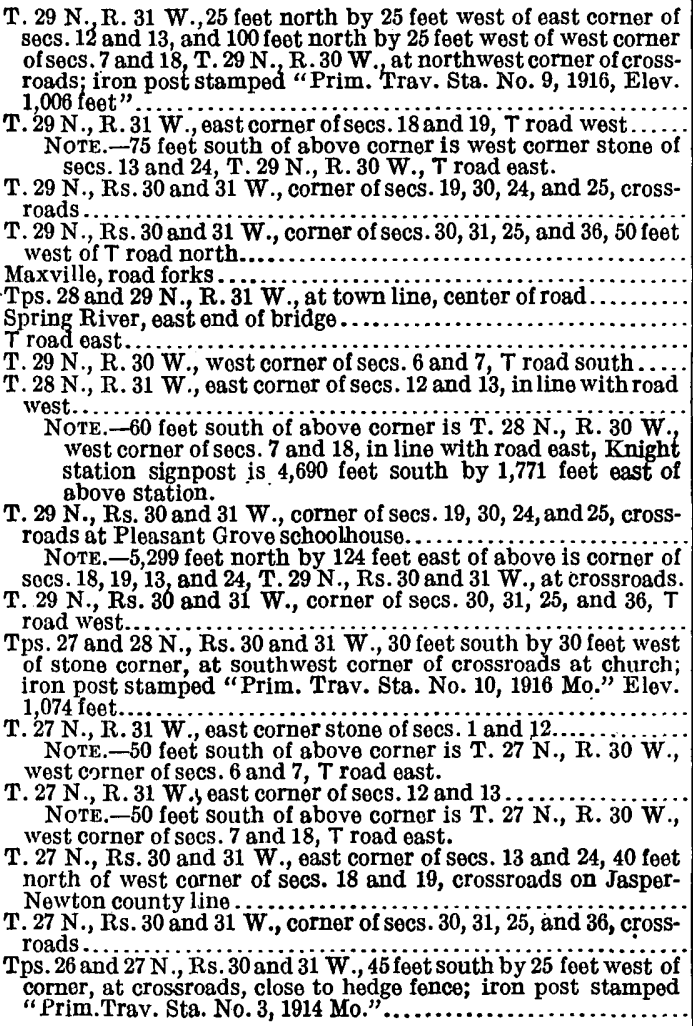 & $\begin{array}{lll}37 & 12 & 47.5 \\
& & \\
37 & 11 & 54.9 \\
37 & 11 & 55.0 \\
37 & 11 & 03.2 \\
37 & 10 & 49.7 \\
37 & 10 & 09.6 \\
37 & 10 & 10.7 \\
37 & 09 & 18.4\end{array}$ & $\begin{array}{llll}94 & 15 & 13.2 \\
& & \\
94 & 15 & 14.8 \\
94 & 16 & 04.1 \\
94 & 16 & 05.8 \\
94 & 16 & 02.7 \\
94 & 16 & 07.4 \\
94 & 15 & 19.0 \\
94 & 15 & 20.7\end{array}$ & $\begin{array}{l}5,322 \\
3,983 \\
5,240 \\
1,382 \\
4,076 \\
3,924\end{array}$ \\
\hline
\end{tabular}

From Knights station west along St. Louis-San Francisco Ry. to State line (1900 traverse line).

T. 28 N., R. 31 W., corner of secs. 11, 12, 13, and 14, 460 feet north of north and south road crossing and 500 feet east of east and west road crossing.

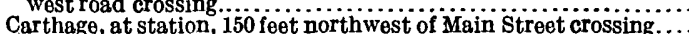

T. 28 N., R. 31 W., quarter corner between secs. 16 and 17, 105

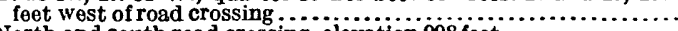

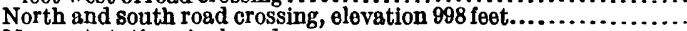
Macy, at station signboard.

Boysur, at north and south road crossing 1,390 foet east of crossroads at north and south road crossing 1 ,300 foot east of crossJoplin Junction, headblock

T. 29 N., R. 33 W., corner of secs. $25,26,35$, and 36,850 feet north of north and south road crossing.

T. 28 N., R. 33 W., north corner of secs. 4 and 5,550 feet north

Missouri Pacific $R$. $R$. crossing on St. Louis-San Francisco $\ldots \ldots \ldots$

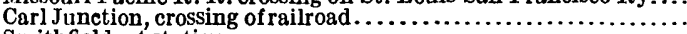

Smithfield, at station.

Missouri-Kansas Stateline crossing on St. Louis-San Francisco $\mathbf{R y}$.

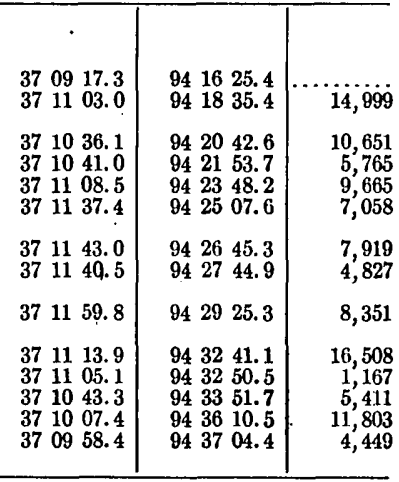

Magnetic declination for north border of quadrangle, $8.4^{\circ} \mathrm{E}$; f for east border of quadrangle, $8.0^{\circ} \mathrm{E}$.; for center line of quadrangle, $8.8^{\circ} \mathrm{E}$, 
JOPIIN DISTRICT QUADRANGLE-Continued.

West along highways near north border of quadrangle.

\begin{tabular}{|c|c|c|c|}
\hline Station. & Latitude. & Longitude. & $\begin{array}{l}\text { Distance } \\
\text { between } \\
\text { stations. }\end{array}$ \\
\hline 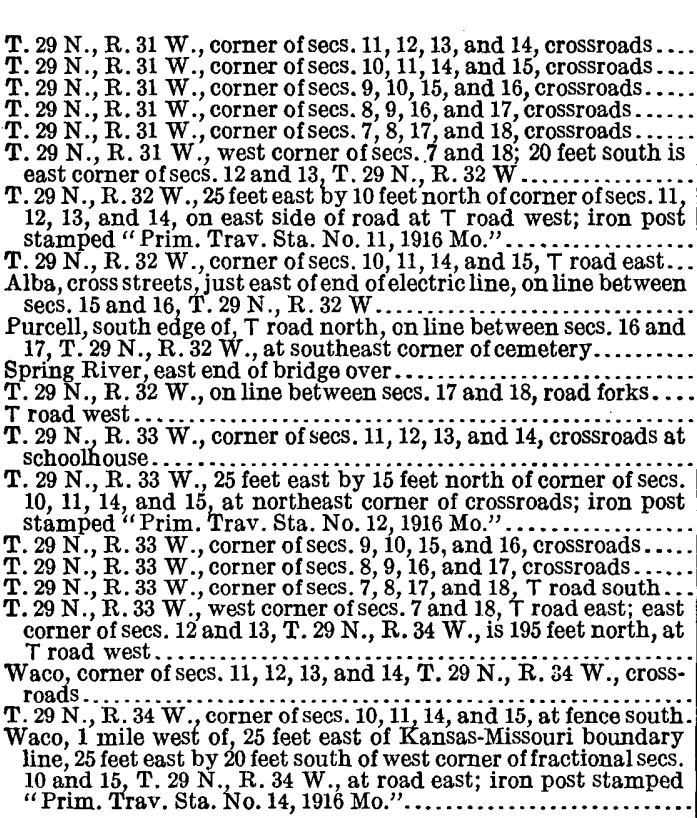 & $\begin{array}{ccc}\circ & \prime & \prime \prime \\
37 & 14 & 31.4 \\
37 & 14 & 30.9 \\
37 & 14 & 31.5 \\
37 & 14 & 31.6 \\
37 & 14 & 31.7 \\
& & \\
37 & 14 & 33.0 \\
& & \\
37 & 14 & 32.0 \\
37 & 14 & 31.8 \\
37 & 14 & 19.5\end{array}$ & $\begin{array}{llll}94 & 26 & 07.7 \\
94 & 27 & 00.9 \\
94 & 27 & 14.1 \\
94 & 28 & 01.7 \\
94 & & \\
94 & 29 & 20.8 \\
& & & \\
94 & 30 & 25.3 \\
94 & 31 & 31.0 \\
94 & 32 & 37.0 \\
94 & 33 & 42.0 \\
& & & \\
94 & 34 & 38.7 \\
& & & \\
94 & 35 & 43.7 \\
94 & 36 & 49.2\end{array}$ & $\begin{array}{r}\text { Feet. } \\
\text { 5, 247 } \\
5,205 \\
5,310 \\
5,275 \\
5,711 \\
5,233 \\
5,337 \\
5,424\end{array}$ \\
\hline
\end{tabular}

Along highways south through center of quadrangle.

T. 29 N., R. 33 W., corner of secs. $14,15,22$, and 23 , crossroads.... T. 29 N., R. 33 W., corner of secs. $22,23,26$, and 27 , crossroads.... T. 29 N., R. 33 W., corner of secs. $26,27,34$, and 35 , crossroads ... T. 29 N., R. 33 W., south corner of secs. 34 and 35, T road north; north corner of secs. 2 and 3 , T. 28 N., R. 33 W., at T road south, is 75 feet east

T. 28 N., R. 33 W., corner of secs. $2,3,10$, and $i 1$, center of north

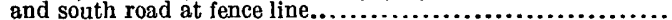

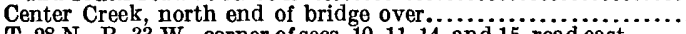
T. 28 N., R. 33 W., corner of secs. $10,11,14$, and 15 , road east. T. 28 N., R. 33 W., corner of secs. 14, 15, 22, and 23 , crossroads.... Joplin, 2 miles north of, on Main Street road, at southeast corner of crossroads, 30 feet south by 20 feet east of corner of secs. 22,23 , 26 and 27:iron post stamped "Prim. Trav. Sta. No. 13, $1916 \mathrm{Mo}$." T. 28 N., R. 33 W., corner of secs. $26,27,34$, and 35 , T road west... Joplin, intersection of Broadway and Main Street.

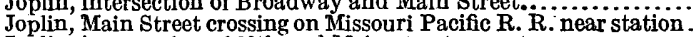
Joplin, intersection of 20 th and Main streets

T. 27 N., R. 33 W., corner of secs. $14,15,22$, and 23, on Jasper

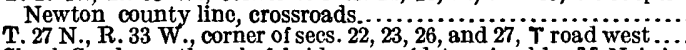
Shoal Creek, north end of bridge over (determined by McNair in 1914)
371346.5 371254.3 3712022

371018.5 371003.2 370926.6 370834.4

$\begin{array}{lll}3707 & 42.0\end{array}$ $\begin{array}{lll}37 & 06 & 50.3\end{array}$ 3705 27. 0 $\begin{array}{lll}37 & 04 & 49.2\end{array}$ 370411.0

370319.0 $\begin{array}{lll}37 & 02 & 27.1\end{array}$ $\begin{array}{lll}37 & 01 & 15.4\end{array}$

\begin{tabular}{|c|c|}
\hline $\begin{array}{lll}94 & 30 & 27.1 \\
94 & 30 & 28.5 \\
94 & 30 & 30.1\end{array}$ & $\begin{array}{l}\ddot{5}, 28 \bar{i} \\
5,270\end{array}$ \\
\hline 943031.7 & 5,265 \\
\hline $\begin{array}{lll}94.30 & 33.3 \\
94 & 30 & 44.6 \\
94 & 30 & 35.3 \\
94 & 30 & 37.6\end{array}$ & $\begin{array}{l}5,232 \\
1,799 \\
3,778 \\
5,280\end{array}$ \\
\hline $\begin{array}{lll}94 & 30 & 39.8 \\
94 & 30 & 42.4 \\
94 & 30 & 46.6 \\
94 & 30 & 48.4 \\
94 & 30 & 49.8\end{array}$ & $\begin{array}{l}\mathbf{5}, 310 \\
\mathbf{5}, \mathbf{2 3 4} \\
\mathbf{8}, \mathbf{4 3 5} \\
\mathbf{3}, 825 \\
\mathbf{3}, 860\end{array}$ \\
\hline $\begin{array}{lll}94 & 30 & 50.6 \\
94 & 30 & 52.8\end{array}$ & $\begin{array}{l}\mathbf{5}, 268 \\
5,248\end{array}$ \\
\hline 943044 & 7,290 \\
\hline
\end{tabular}




\section{SARCOXIE QUADRANGLE.}

South along highways near north half of east border of quadrangle. (By J. H. Wilson in 1916.)

\begin{tabular}{|c|c|c|c|}
\hline Station. & Latitude. & Longitude. & $\begin{array}{l}\text { Distance } \\
\text { between } \\
\text { stntions. }\end{array}$ \\
\hline 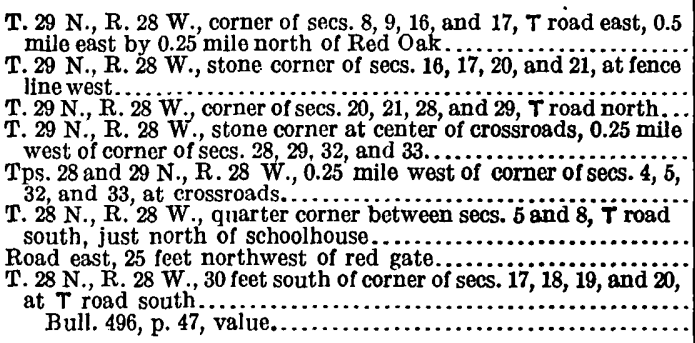 & $\begin{array}{cccc}\circ & \prime & \prime \prime \\
37 & 14 & 09.6 \\
& & \\
37 & 13 & 17.2 \\
37 & 12 & 25.0 \\
& & & \\
37 & 11 & 33.1 \\
& & & \\
37 & 10 & 40.9 \\
37 & 09 & 47.6 \\
37 & 08 & 48.2 \\
37 & 08 & 03.9 \\
37 & 08 & 07.1\end{array}$ & $\begin{array}{cccc}0 & \prime & \prime \prime \\
94 & 00 & 00.2 \\
94 & 00 & 01.3 \\
94 & 00 & 02.4 \\
94 & 00 & 19.8 \\
94 & 00 & 20.8 \\
94 & 00 & 38.0 \\
94 & 00 & 39.3 \\
94 & 01 & 13.0 \\
94 & 01 & 14.0\end{array}$ & $\begin{array}{r}\text { Feet. } \\
\ldots \ldots . . . \\
5,296 \\
5,281 \\
5,441 \\
5,286 \\
5,568 \\
6,008 \\
5,249 \\
\ldots \ldots . . .\end{array}$ \\
\hline
\end{tabular}

Near northwest corner of quadrangle.

T. 29 N., R. 30 W., stone corner of secs. $7,8,17$, and 18, crossroads at schoolhouse.
371430.5

941403.4

From Reeds station along St. Louis-San Francisco Ry. to Knights. (1800 traverse line.)

Reeds, at station (Bull. 496, corrected to approximate North A American datum) 28 N., R. 30 W., corner of sees. $17,18,19$, and 20,30 feet south of T. $28 \mathrm{~N} ., \mathrm{R} .30 \mathrm{~W}$, corner of secs. $17,18,19$, and 20,30 feet south of Knights, at station (tied by 1916 traverse); north and south road crossing is 63 feet north by 308 feet west.

\begin{tabular}{|c|c|}
\hline $\begin{array}{lll}37 & 07 & 02.8\end{array}$ & 941003.5 \\
\hline $\begin{array}{lll}37 & 08 & 23.9\end{array}$ & . 941414.0 \\
\hline 70832.1 & 941458.9 \\
\hline
\end{tabular}

Magnetic declination for north half of east border of quadrangle, $7.6^{\circ} \mathrm{E}$.; for west border of quadrangle, $8.0^{\circ} \mathrm{E}$; f for north border of quadrangle, $7.7^{\circ} \mathrm{E}$.

From Wentworth station northwest along St. Louis-San Francisco Ry. to Reeds. (From Bull. 181, page 170, corrected; 1000 traverse line.)

\begin{tabular}{|c|c|c|c|}
\hline 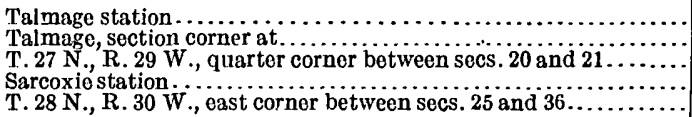 & $\begin{array}{lll}37 & 00 & 54.4 \\
37 & 01 & 12.0 \\
37 & 02 & 32.0 \\
37 & 03 & 59.5 \\
37 & 06 & 31.0\end{array}$ & $\begin{array}{lll}94 & 05 & 34.9 \\
94 & 05 & 45.0 \\
94 & 06 & 47.2 \\
94 & 07 & 00.0 \\
94 & 08 & 50.4\end{array}$ & $\begin{array}{r}1,955 \\
9,525 \\
8,904 \\
17,736\end{array}$ \\
\hline
\end{tabular}


SPRINGFIELD QUADRANGLE.

North along highways near west border of quadrangle. (By J. H. Wilson in 1916.)

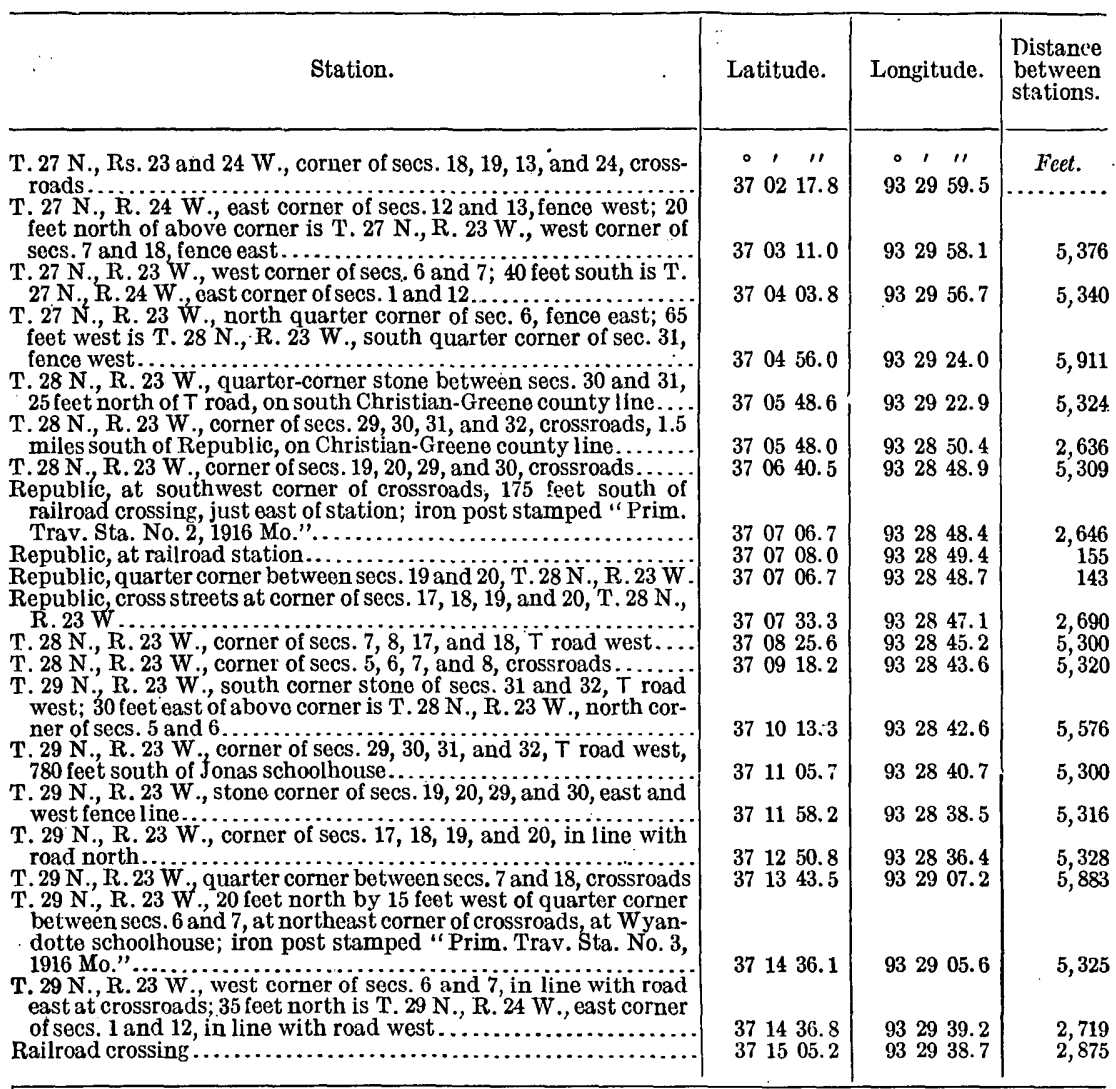

Along St. Louis-San Francisco Ry.

\begin{tabular}{|c|c|c|c|}
\hline 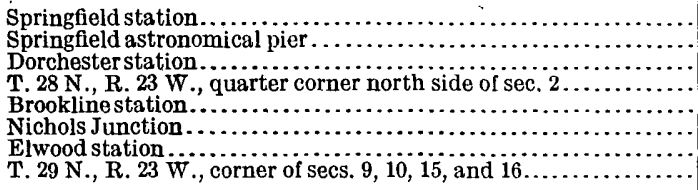 & $\begin{array}{lll}37 & 13 & 50.4 \\
37 & 13 & 12.8 \\
37 & 12 & 33.3 \\
37 & 10 & 08.8 \\
37 & 09 & 47.1 \\
37 & 13 & 07.1 \\
37 & 13 & 32.2 \\
37 & 13 & 40.1\end{array}$ & $\begin{array}{lll}93 & 17 & 12.4 \\
93 & 17 & 13.2 \\
93 & 23 & 09.1 \\
93 & 24 & 52.7 \\
93 & 25 & 08.7 \\
93 & 21 & 48.7 \\
93 & 25 & 51.7 \\
93 & 26 & 23.9\end{array}$ & 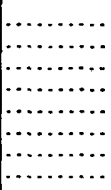 \\
\hline
\end{tabular}

Magnetic declination (1916) for west border of quadrangle, $7.5^{\circ} \mathrm{E}$. 
STOTTS CITY QUADRANGLE.

South along highways near east border of quadrangle. (By J. H. Wilson in 1916.)

\begin{tabular}{|c|c|c|c|}
\hline Station. & Latitude. & Longitude. & $\begin{array}{l}\text { Distanco } \\
\text { between } \\
\text { stations. }\end{array}$ \\
\hline 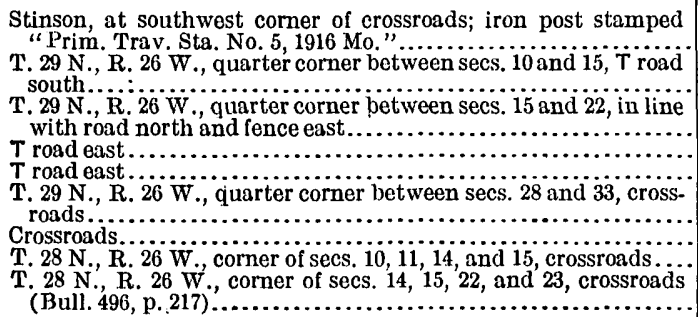 & $\begin{array}{ccc}\circ & 1 & \prime \prime \\
37 & 14 & 47.5 \\
37 & 13 & 55.4 \\
37 & 13 & 02.9 \\
37 & 12 & 23.7 \\
37 & 11 & 58.6 \\
37 & 11 & 19.1 \\
37 & 10 & 52.2 \\
37 & 08 & 40.8 \\
37 & 07 & 48.3\end{array}$ & \begin{tabular}{lcc|}
$\circ$ & \multicolumn{1}{c}{} & $\prime \prime$ \\
93 & 45 & 08.3 \\
93 & 45 & 26.0 \\
93 & 45 & 27.4 \\
93 & 45 & 28.2 \\
93 & 46 & 34.8 \\
93 & 46 & 35.8 \\
93 & 45 & 46.8 \\
93 & 45 & 00.8 \\
93 & 45 & 01.8
\end{tabular} & $\begin{array}{r}\text { Feet. } \\
5,457 \\
5,310 \\
3,968 \\
5,960 \\
3,991 \\
4,809 \\
\\
5,316\end{array}$ \\
\hline
\end{tabular}

Magnetic declination for north half of east border of quadrangle, $7.4^{\circ} \mathrm{E}$.; for north border of quadrangle, $7.3^{\circ} \mathrm{E}$.; for north half of west border of quadrangle, $7.6^{\circ} \mathrm{E}$.

\section{MISSOURI, KANSAS, AND NEBRASKA.}

PRIMARY TRAVERSE.

Atchison, Hiawatha, Maitland, Rulo, Skidmore, and Tarkio quadrangles.

HOLT COUNTY, MO.; BROWN COUNTY, KANS.; RICHARDSON COUNTY, NEBR.

The following geographic positions, on approximate North American datum, were determined by J. H. Wilson in 1916 and are based on primary traverse positions described in Bulletin 644 and upon Missouri River Commission triangulation stations Craig and Napier.

For other positions in Kansas see U. S. Geological Survey bulletins $122,181,216,245,440$, and 644 , U. S. Coast and Geodetic Survey Report for 1902, and Missouri River Commission reports for 1887, 1890 , and 1891.

ATCHISON QUADRANGLE, KANS.

East along highways near north border of quadrangle. (By J. H. Wilson in 1916.)

\begin{tabular}{|c|c|c|c|}
\hline Station. & Iatitude. & Jongitude. & $\begin{array}{l}\text { Distance } \\
\text { between } \\
\text { stations. }\end{array}$ \\
\hline 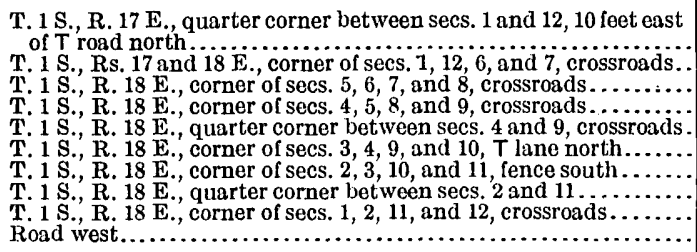 & $\begin{array}{lcc}\circ & \prime \prime & \\
39 & 59 & 10.8 \\
39 & 59 & 10.8 \\
39 & 59 & 11.2 \\
39 & 59 & 11.8 \\
39 & 59 & 12.0 \\
39 & 59 & 12.2 \\
39 & 59 & 12.7 \\
39 & 59 & 13.0 \\
39 & 59 & 13.6 \\
39 & 59 & 13.6\end{array}$ & $\begin{array}{ccc}\circ & \prime & \prime \prime \\
95 & 27 & 40.1 \\
95 & 27 & 06.0 \\
95 & 26 & 01.4 \\
95 & 24 & 53.6 \\
95 & 24 & 19.7 \\
95 & 23 & 45.9 \\
95 & 22 & 38.1 \\
95 & 22 & 04.2 \\
95 & 21 & 30.3 \\
95 & 20 & 56.7\end{array}$ & $\begin{array}{r}\text { Feet. } \\
\ldots \ldots \ldots \\
2,660 \\
5,032 \\
5,280 \\
2,635 \\
2,640 \\
5,280 \\
2,680 \\
2,598 \\
2,616\end{array}$ \\
\hline
\end{tabular}

Magnetic declination for north border of quadrangle, $11^{\circ} 17^{\prime} \mathrm{E}$.

East along Nebraska-Kansas State line: (By J. H. Wilson In 1816.)

T. 1 S., R. 17 E., north corner of secs. 2 and 3, T road south. T. 1 N., R. 17 E., south corner of secs. 34 and 35 , T road north... T. 1 S., R. 17 E., north corner of secs. 1 and 2 , church, T road south. Road east.

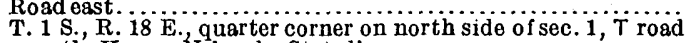
south, Kansas-Nebraska State line...

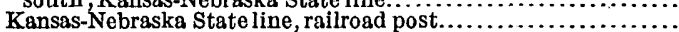

395959.6 395959.5 395959.3 395958.5

395959.2 400000.0

\begin{tabular}{rrr|r}
95 & 29 & 23.4 & \\
95 & 28 & 56.9 & $\ldots \ldots$ \\
95 & 28 & 15.3 & 2,070 \\
95 & 27 & 39.9 & 3,235 \\
& & 2,763 \\
95 & 20 & 56.6 & \\
95 & 19 & 52.1 & $\ldots \ldots, \ldots$
\end{tabular}


HIAWATHA QUADRANGLE, KANS.-NEBR.

Along Nebraska-Kansas State line. (By J. H. Wilson in 1916.)

\begin{tabular}{|c|c|c|c|}
\hline Station. & Latitude. & Longitude. & $\begin{array}{l}\text { Distance } \\
\text { between } \\
\text { stations. }\end{array}$ \\
\hline $\begin{array}{l}\text { Preston, } 2 \text { miles south by } 0.3 \text { mile west of, } 280 \text { feet east of north } \\
\text { corner of secs. } 4 \text { and } 5 \text {, T. } 1 \mathrm{~S} \text {., R. } 17 \mathrm{E} \text {. on north side of State } \\
\text { line road, in sjuth foundation wall of barn; aluminum tablet } \\
\text { stamped "1028 Prim. Trav. Sta. No. 22, Nebr. 1913"............. } \\
\text { T. } 1 \text { S., R. } 17 \text { E., north corner of secs. } 3 \text { and 4, T road south...... } \\
\text { T. } 1 \text { N., R. } 17 \text { E., south corner of secs. } 33 \text { and } 34 \text {, T road north.... }\end{array}$ & $\begin{array}{ccc}\circ & \prime & \prime \prime \\
40 & 00 & 02.4 \\
39 & 59 & 59.6 \\
39 & 59 & 59.6\end{array}$ & $\begin{array}{ccc}\circ & \prime & \prime \prime \\
95 & 31 & 35.1 \\
95 & 30 & 30.6 \\
95 & 30 & 04.6\end{array}$ & $\begin{array}{l}\text { Feet. } \\
.5,028 \\
2,030\end{array}$ \\
\hline
\end{tabular}

Magnetic declination for southeast corner of quadrangle, $9^{\circ} 55^{\prime} \mathrm{E}$.

RULO QUADRANGLE, NEBR.

West along Burlington Route and highways in south part of quadrangle. (By J. H. Wilson in 1916.)

\begin{tabular}{|c|c|c|c|}
\hline Station. & Latitude. & Longitude. & $\begin{array}{l}\text { Distance } \\
\text { between } \\
\text { stations. }\end{array}$ \\
\hline 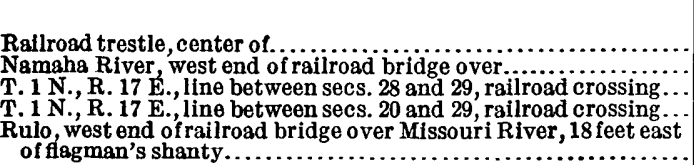 & $\begin{array}{ccc}\circ & \prime & \prime \prime \\
40 & 01 & 28.0 \\
40 & 01 & 33.6 \\
40 & 01 & 26.0 \\
40 & 01 & 42.4 \\
40 & & \\
40 & 12.9\end{array}$ & $\begin{array}{ccc}\circ & \prime & \prime \prime \\
95 & 21 & 31.9 \\
95 & 22 & 58.4 \\
95 & 24 & 24.6 \\
95 & 25 & 26.8 \\
95 & 25 & 23.8\end{array}$ & 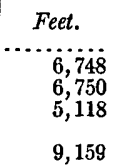 \\
\hline
\end{tabular}

Magnetic declination for south border of quadrangle, $8^{\circ} 57^{\prime} \mathrm{E}$.

MAITLAND QUADRANGLE, Mo.

Forth along highways near west border of quadrangle. (By J. H. Wilson in 1916.)

\begin{tabular}{|c|c|c|c|}
\hline Station. & Latitude. & Longitude. & $\begin{array}{l}\text { Distance } \\
\text { between } \\
\text { stations. }\end{array}$ \\
\hline 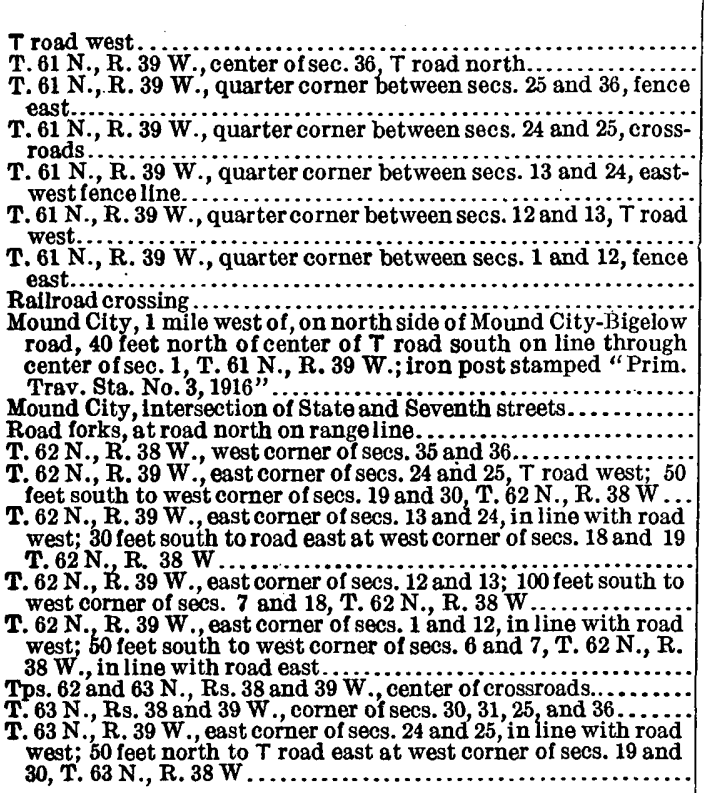 & $\begin{array}{ccc}\circ & \prime & \prime \prime \\
40 & 02 & 53.6 \\
40 & 03 & 06.9 \\
40 & 03 & 33.0 \\
40 & 04 & 25.2 \\
40 & 05 & 17.5 \\
40 & 06 & 10.1 \\
40 & 07 & 02.3 \\
40 & 07 & 21.8\end{array}$ & $\begin{array}{ccc}\circ & \prime & \prime \prime \\
95 & 14 & 45.0 \\
95 & 14 & 55.7 \\
95 & 14 & 55.7 \\
95 & 14 & 55.7 \\
95 & 14 & 55.2\end{array}$ & $\begin{array}{r}\text { Feet. } \\
\cdots \\
1,580 \\
2,635 \\
5,290 \\
5,295\end{array}$ \\
\hline
\end{tabular}

Magnetic declination along west border of quadrangle, $9^{\circ} 45^{\prime} \mathrm{E}$. 
RULO QUADRANGLE, MO.

Along highways and Chicago, Burlington \& Quincy R. R. in north part of quadra ngle. (By J. H. Wilson in 1916.)

\begin{tabular}{|c|c|c|c|}
\hline Station. & Latitude. & Longitude. & $\begin{array}{l}\text { Distance } \\
\text { between } \\
\text { stations. }\end{array}$ \\
\hline 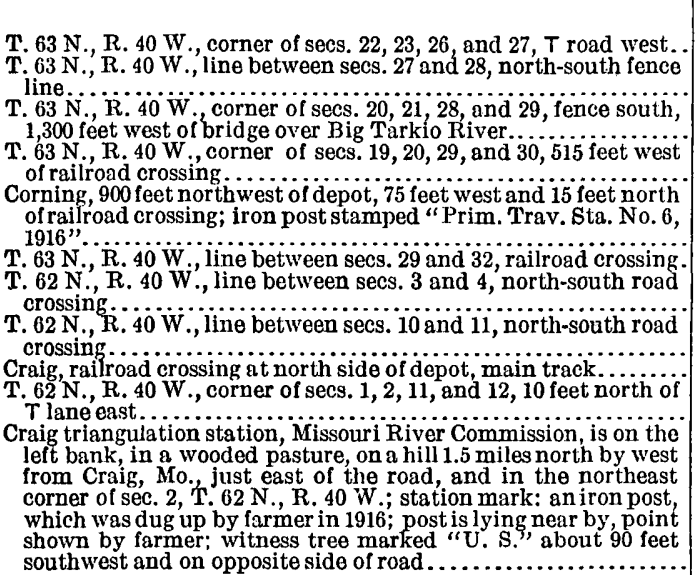 & $\begin{array}{ccc}\circ & \prime & \prime \prime \\
40 & 14 & 54.8 \\
40 & 14 & 42.1 \\
40 & 14 & 56.5 \\
40 & 14 & 57.4 \\
40 & 14 & 57.8 \\
40 & 14 & 04.5 \\
40 & 12 & 59.7\end{array}$ & $\begin{array}{ccc}\circ & \prime \prime & \prime \prime \\
95 & 23 & 20.4 \\
95 & 24 & 28.6 \\
95 & 25 & 38.6 \\
95 & 26 & 47.1 \\
95 & 27 & 24.3 \\
95 & 26 & 08.3 \\
95 & 24 & 36.0\end{array}$ & $\begin{array}{r}\text { Feet. } \\
\ldots \ldots \ldots \\
5,440 \\
5,620 \\
5,311\end{array}$ \\
\hline
\end{tabular}

East along Burlington Route and highways in southeast part of quadrangle.

Missouri River, east end of railroad bridge over.

T. 61 N., R. 40 W., line between secs. 26 and 27 , north-south road

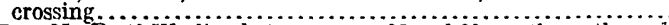
T. 61 N., R. 40 W., line between secs. 35 and 36 , north-south road crossing.

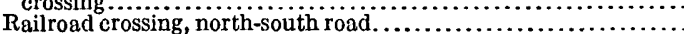

T. 61 N., Rs. 39 and 40 W., probably on line between, center of trestle $5-19$, in line with fence north through lake............

T. 61 N., R. 39 W., line between secs. 31 and 32 , north-south road

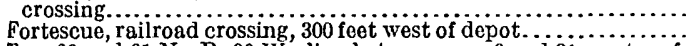

Tps, 60 and 61 N., R. 39 W., line between secs. 6 and 31 , center of

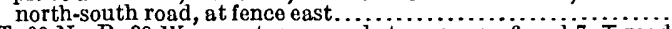
T. 60 N., R. 39 W., quarter corner between secs. 6 and $7,{ }^{\prime}$ road

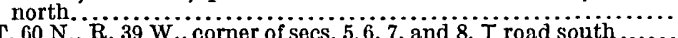
T. 60 N., R. 39 W., line between secs. 17 and 18, telephone polo at

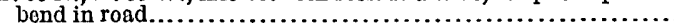

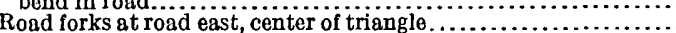

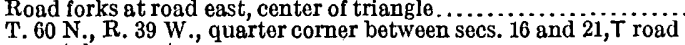

T. 60 N., R. 39 W., quarter corner between secs. 15 and 22 , center of north-south road, in line with fence east, 980 feet south of

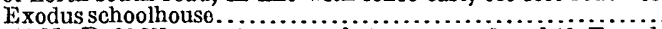
T. 60 N., R. 39 W., quarter corner between secs. 3 and 10 , T road

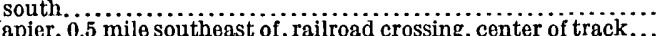

Napier triangulation station is on the leftbank, on a narrow, bare ridge, three-fourths of a mile east-south-east of Napier railroad station, and just east of the graveyard on the hillside; station

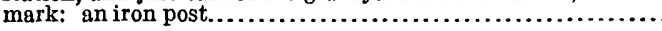

Magnetic declination for south border of quadrangle, $8^{\circ} 57^{\prime} \mathrm{E}$.; for north part of quadrangle, $9^{\circ} 24^{\prime} \mathrm{E}$.

\begin{tabular}{l|lll|rr|r}
40 & 03 & 18.8 & 95 & 24 & 58.8 & $\ldots \ldots \ldots$ \\
40 & 03 & 39.5 & 95 & 23 & 30.1 & 7,215 \\
40 & 03 & 19.5 & 95 & 22 & 22.5 & 5,634 \\
40 & 03 & 11.7 & 55 & 21 & 48.0 & 2,794 \\
40 & 03 & 11.4 & 55 & 21 & 11.7 & 2,830 \\
40 & 03 & 10.6 & 95 & 20 & 00.9 & 5,505 \\
40 & 03 & 10.1 & 95 & 19 & 08.9 & 4,050 \\
40 & 02 & 43.5 & 95 & 19 & 12.2 & 2,708 \\
40 & 02 & 33.1 & 95 & 19 & 12.3 & 1,050 \\
40 & 02 & 32.7 & 95 & 18 & 38.4 & 2,640 \\
40 & 01 & 22.8 & 95 & 18 & 39.4 & 7,080 \\
40 & 00 & 47.9 & 95 & 17 & 55.8 & 4,893 \\
40 & 00 & 47.8 & 95 & 16 & 58.6 & 4,452 \\
& & & & & \\
40 & 00 & 47.9 & 95 & 15 & 51.3 & 6,570 \\
40 & 02 & 31.9 & 95 & 15 & 50.4 & 10,525 \\
40 & 02 & 31.5 & 95 & 15 & 05.2 & 3,515 \\
& & & & \\
40 & 02 & 47.22 & 95 & 14 & 35.89 & 2,783
\end{tabular}

7,215

2,794

830

050

708

640

080

452

570

10,525

2,783 


\section{ATCHISON QUADRANGLE, MO.}

(By J. H. Wilson in 1916.)

\begin{tabular}{|c|c|c|c|}
\hline Station. & Latitude. & Longitude. & $\begin{array}{l}\text { Distance } \\
\text { between } \\
\text { stations. }\end{array}$ \\
\hline 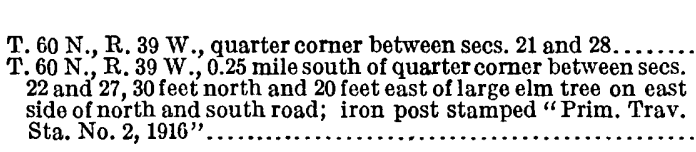 & $\begin{array}{ccc}\circ & \prime & \prime \prime \\
39 & 59 & 55.6\end{array}$ & $\begin{array}{cccc}\circ & \prime & \prime \prime \\
95 & 16 & 59.1 \\
95 & 15 & 51.3\end{array}$ & Feet. \\
\hline
\end{tabular}

SKIDMORE QUADRANGLE, MO.

In southwest portion of quadrangle. (By J. H. Wilson in 1916.)

\begin{tabular}{|c|c|c|c|}
\hline Station. & Latitude. & Longitude. & $\begin{array}{l}\text { Distance } \\
\text { between } \\
\text { stations. }\end{array}$ \\
\hline $\begin{array}{l}\text { T. } 63 \text { N., R. } 39 \text { W., east corner of secs. } 13 \text { and } 24,45 \text { feet north and } \\
18 \text { feet east of, and T. } 63 \text { N., R. } 38 \text { W., } 15 \text { feet north and } 18 \text { feet } \\
\text { east of west corner of secs. } 18 \text { and } 19 \text {, at northeast corner of cross- } \\
\text { roads, on north side of road, on A tchison-Holt county line; iron } \\
\text { post stamped "Prim. Trav. Sta. No. 4, 1916"................. }\end{array}$ & $\begin{array}{ccc}\circ & \prime & \prime \prime \\
40 & 15 & 46.2\end{array}$ & $\begin{array}{ccc}\circ & \prime \prime \\
95 & 14 & 14.3\end{array}$ & Fcet. \\
\hline
\end{tabular}

TARKIO QUADRANGLE, MO.

West along highways near south border of quadrangle. (By J. H. Wilson in 1916.)

\begin{tabular}{|c|c|c|c|}
\hline Station. & Latitude. & Longitude. & $\begin{array}{l}\text { Distance } \\
\text { between } \\
\text { stations. }\end{array}$ \\
\hline 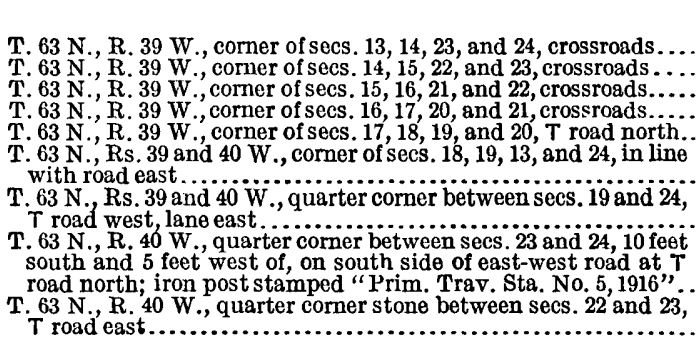 & $\begin{array}{ccc}\circ & \prime & \prime \prime \\
40 & 15 & 46.0 \\
40 & 15 & 45.9 \\
40 & 15 & 45.6 \\
40 & 15 & 45.8 \\
40 & 15 & 45.8 \\
40 & 15 & 46.2 \\
40 & 15 & 20.1\end{array}$ & \begin{tabular}{ccc}
$\circ$ & $\prime$ & \multicolumn{1}{l}{} \\
95 & 15 & 22.8 \\
95 & 16 & 31.5 \\
95 & 17 & 39.9 \\
95 & 18 & 48.6 \\
95 & 19 & 58.6 \\
95 & 21 & 03.1 \\
95 & 21 & 03.4
\end{tabular} & $\begin{array}{r}\text { Feet. } \\
\text { 5, 320 } \\
5,310 \\
5,325 \\
5,425 \\
5,005 \\
2,640\end{array}$ \\
\hline
\end{tabular}

Magnetic declination along east part of south border of quadrangle, $9^{\circ} 19^{\prime} \mathrm{E}$.

\section{NEBRASKA AND IOWA.}

\section{PRIMARY TRAVERSE.}

\section{FORT OMAHA BALLOON FIELD.}

Arlington, Ascot, Blair, Calhoun, Chalco, Elkhorn, Florence, Irvington, Kennard,

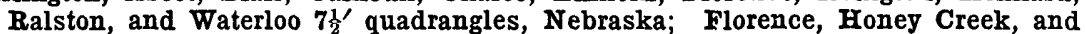
Omaha vicinity quadrangles, Iowa.

dogglas and washington counties, nebr., pottawattamie codnty, iowa.

The following geographic positions on approximate North American datum were determined by Oscar Jones, in 1917, and are based on positions of the Missouri River Commission triangulation stations near Omaha. 
For other positions in Nebraska see U. S. Geological Survey Bulletins 201, 276, and 644, Part I of the Eighteenth and Nineteenth Annual Reports, Special Publication No. 19 of the U. S. Coast and Geodetic Survey, and Annual Reports for 1901 and 1911 of the latter Survey; see also Reports of the Missouri River Commission for 1891 and 1893.

For other positions in Iowa see U. S. Geological Survey Bulletins 181, 201, 276, 440, 496, 551, and 644, and Eighteenth Annual Report, Part I, Reports of the Missouri River Commission for 1891 and 1893, and Reports of the Mississippi River Commission for 1893, 1894, and 1895.

ARIINGTON QUADRANGLE, NEBR.

West and south along highways and section lines. (By Oscar Jones in 1917.)

\begin{tabular}{|c|c|c|c|}
\hline Station. & Latitude. & Longitude. & $\begin{array}{l}\text { Distance } \\
\text { between } \\
\text { stations. }\end{array}$ \\
\hline 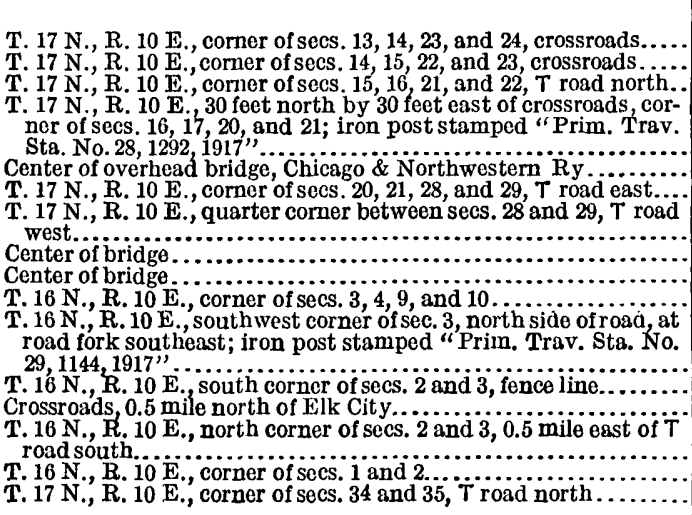 & $\begin{array}{ccc}\circ & \prime & \prime \prime \\
41 & 26 & 11.7 \\
41 & 26 & 12.0 \\
41 & 26 & 11.9 \\
& & \\
& & \\
41 & 26 & 12.2 \\
41 & 25 & 46.3 \\
41 & 25 & 19.6 \\
& & \\
41 & 24 & 53.6 \\
41 & 24 & 33.3 \\
41 & 23 & 39.2 \\
41 & 22 & 43.8\end{array}$ & \begin{tabular}{ccc}
$\circ$ & $\prime$ & \multicolumn{1}{l}{} \\
96 & 15 & 07.3 \\
96 & 16 & 16.8 \\
96 & 17 & 26.2 \\
& & \\
96 & 18 & 34.8 \\
96 & 18 & 35.4 \\
96 & 18 & 35.4 \\
96 & 18 & 35.3 \\
96 & 18 & 26.5 \\
96 & 17 & 52.0 \\
96 & 17 & 32.7
\end{tabular} & $\begin{array}{r}\text { Feet. } \\
\ldots . \\
5,290 \\
5,285 \\
\\
5,231 \\
2,627 \\
2,700 \\
2,625 \\
2,170 \\
6,069 \\
5,802\end{array}$ \\
\hline
\end{tabular}

ASCOT QUADRANGLE, NEBR.

Southeast along highways. (By Oscar Jones in 1917.)

\begin{tabular}{|c|c|c|c|}
\hline Station. & Latitude. & Longitude. & $\begin{array}{l}\text { Distance } \\
\text { between } \\
\text { stations. }\end{array}$ \\
\hline 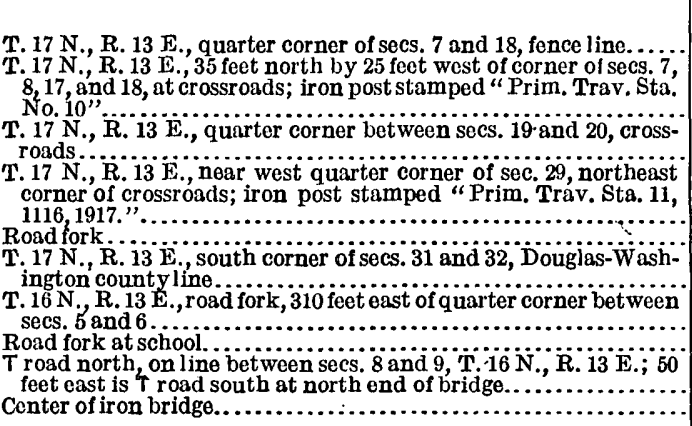 & $\begin{array}{lll}41 & 24 & 54.1 \\
41 & 23 & 38 . \\
& & \\
41 & 23 & 34.7 \\
& & \\
41 & 23 & 16.2 \\
41 & 22 & 30.9 \\
& & \\
41 & 22 & 35.6 \\
41 & 22 & 36.0\end{array}$ & $\begin{array}{llll}95 & 58 & 58.6 \\
95 & 58 & 58.5 \\
& & \\
95 & 58 & 59.0 \\
& & & \\
95 & 59 & 03.6 \\
95 & 58 & 36.6 \\
& & & \\
95 & 57 & 58.9 \\
95 & 57 & 24.0\end{array}$ & $\begin{array}{r}\text { Feet. } \\
2,625 \\
7,974\end{array}$ \\
\hline
\end{tabular}

Magnetic declination for southeast quarter of quadrangle, $8.9^{\circ} \mathrm{E}$. 
ASCOT QUADRANGLE, NEBR.-Continued.

Along Chicago, St. Paul, Minneapolis \& Omaha Ry. In southwest part of quadrangle.

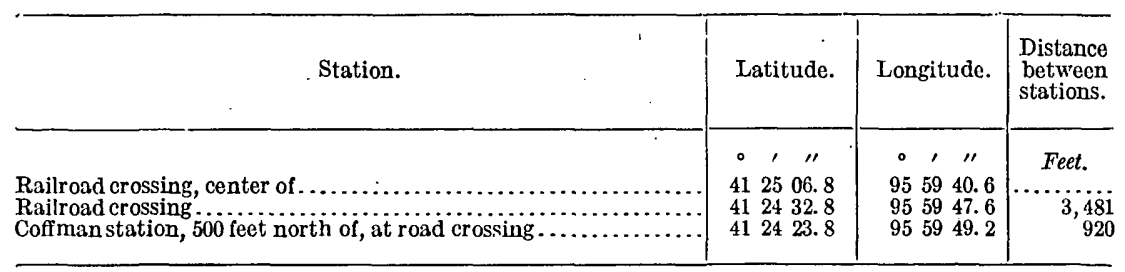

West along highways in south part of quadrangle.

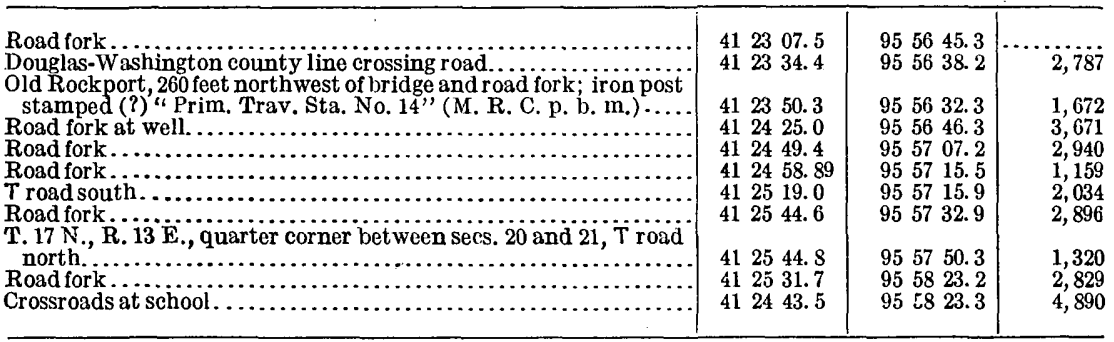

Magnetic declination for southwest quarter of quadrangle, $9.1^{\circ} \mathrm{E}$.

BLAIR QUADRANGLE, NEBR.

Northwest along highways. (By Oscar Jones in 1917.)

\begin{tabular}{|c|c|c|c|}
\hline Station. & Latitude. & Longitude. & $\begin{array}{l}\text { Distance } \\
\text { between } \\
\text { stations. }\end{array}$ \\
\hline & $\circ, 11$ & $\circ, 11$ & Feet. \\
\hline $\begin{array}{l}\text { Crossroads, } 100 \text { feet west is corner of section } \ldots \ldots \ldots \ldots \ldots \ldots \ldots \\
T \text { road west (Blair triangulation station not found) } \ldots \ldots \ldots \ldots \ldots \ldots\end{array}$ & $\begin{array}{lll}41 & 30 & 32.3 \\
41 & 30 & 58.4\end{array}$ & $\begin{array}{lll}96 & 06 & 58.3 \\
96 & 07 & 32.4\end{array}$ & 3,707 \\
\hline $\begin{array}{l}\text { Blair, } 1 \text { mile south of, near center of sec. } 13, \mathbf{T}, 18 \mathrm{~N}, \mathrm{R} .11 \mathrm{E} \text {, at } \\
\text { southwest corner of T road south, road fork; iron post stamped } \\
\text { "Prim. Trav. Sta. No. } 7,1120,1917 \text { " }\end{array}$ & A2 00000 & 960735.4 & 0,100 \\
\hline 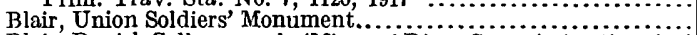 & 413226.3 & 960809.6 & 4,810 \\
\hline Blair, Danish College cupola (Missouri River Commission tie point) & 413303.16 & 960922.06 & 6,656 \\
\hline
\end{tabular}

North to base line.

T. 18 N., R. 11 E., quarter corner between secs. 1 and 12 , crossroads Tps. 18 and 19 N., R. 11 E., quarter corner between sees. 1 and 36 ,

T. 19 N., R. 11 E., quarter corner between secs. 25 and 36 , crossroads T. 19 N., R. 11 E., center of sec. 25 , crossroads................... T. 19 N., $R .11 \mathrm{E}$., quarter corner between secs. 24 and $25, T$ road east. Blair, 5 miles north, on base line, 3,417 feet west of East Base station, on east side of north-south road, 0.25 mile north of south quarter post of sec. 14, T. 19 N., R. 11 E., 0.25 mile south of W. Tyson's house; copper bolt in tile surmounted by iron pipe, iron post (Mo. R. Comm. b. m. 128/2)...

Copper bolt, elev., 1,013.41 feet.

Cap on pipe, elev., 1,017.49 feet.

Blair, East Base, Missouri River Commission station, on right bank, 0.25 mile southeast from W. Tyson's house, near center of SW. 1 sec. 18, T. 19 N., R. 12 E. (not found in 1917)............

\begin{tabular}{|c|c|c|}
\hline $\begin{array}{lll}41 & 33 & 08.8\end{array}$ & $\begin{array}{lll}96 & 07 & 34.9\end{array}$ & .. \\
\hline $\begin{array}{lll}41 & 34 & 01.0 \\
41 & 34 & 53.6 \\
41 & 35 & 19.7 \\
41 & 35 & 45.7\end{array}$ & $\begin{array}{lll}96 & 07 & 34.8 \\
96 & 07 & 34.8 \\
96 & 07 & 34.7 \\
96 & 07 & 34.6\end{array}$ & $\begin{array}{l}5,290 \\
5,320 \\
2,650 \\
2,630\end{array}$ \\
\hline 413651.4 & $\begin{array}{lll}96 & 07 & 33.9\end{array}$ & 6,649 \\
\hline $41 \quad 36 \quad 51.41$ & $9606 \quad 48.83$ & 3,417 \\
\hline
\end{tabular}

Southeast along highways.

Road fork

Road fork, road west on section line

Road fork

Road fork. 
BLAIR QUADRANGLE, NEBR.-Continued.

Southeast along highways near south border of quadrangle. (By Oscar Jones in 1917.)

\begin{tabular}{|c|c|c|c|}
\hline Station. & Latitude. & Longitude. & $\begin{array}{l}\text { Distance } \\
\text { between } \\
\text { stations. }\end{array}$ \\
\hline 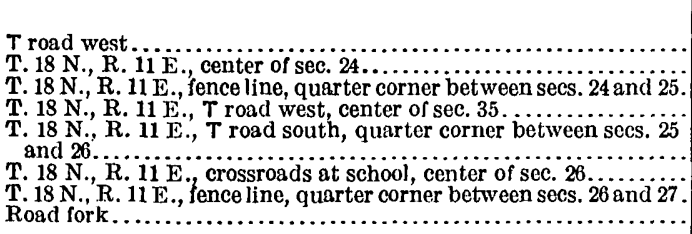 & $\begin{array}{ccc}\circ & \prime & \prime \prime \\
41 & 30 & 58.4 \\
41 & 30 & 58.2 \\
41 & 30 & 32.2 \\
41 & 30 & 06.0 \\
& & \\
41 & 30 & 06.0 \\
41 & 30 & 06.0 \\
41 & 30 & 06.2 \\
41 & 30 & 06.2\end{array}$ & $\begin{array}{ccc}\circ & \prime & \prime \prime \\
96 & 07 & 32.4 \\
96 & 07 & 35.1 \\
96 & 07 & 35.4 \\
96 & 07 & 35.6 \\
& & \\
96 & 08 & 10.0 \\
96 & 08 & 44.8 \\
96 & 09 & 19.6 \\
96 & 09 & 34.4\end{array}$ & $\begin{array}{r}\text { Fect. } \\
\ldots \ldots . .10 \\
210 \\
2,630 \\
2,652 \\
\\
2,620 \\
2,650 \\
2,650 \\
1,119\end{array}$ \\
\hline
\end{tabular}

CALHOUN QUADRANGLE, NEBR.

Northeast along highways. (By Oscar Jones in 1917.)

\begin{tabular}{|c|c|c|c|}
\hline Station. & Latitude. & Longitude. & $\begin{array}{l}\text { Distance } \\
\text { between } \\
\text { stations. }\end{array}$ \\
\hline 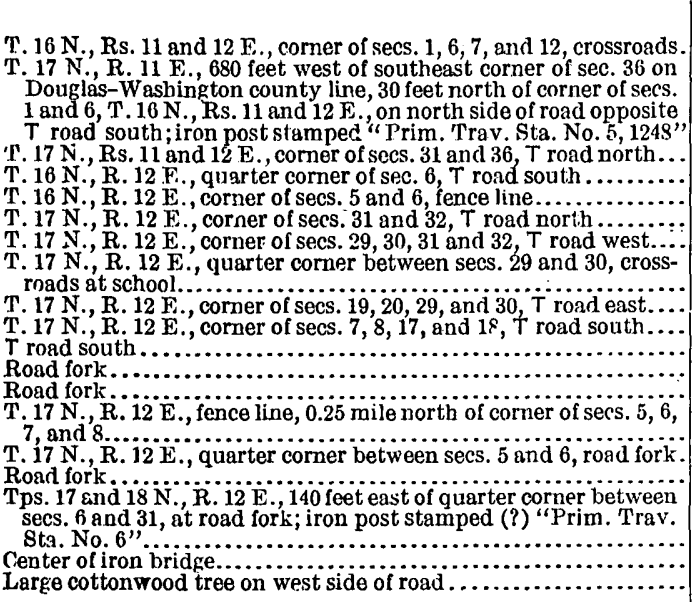 & $\begin{array}{lll}41 & 23 & 35.2 \\
41 & 23 & 34.9 \\
41 & 23 & 35.0 \\
41 & 23 & 35.1 \\
41 & 23 & 35.1 \\
41 & 24 & 27.2 \\
& & \\
41 & 24 & 53.2 \\
41 & 25 & 19.4 \\
41 & 27 & 03.4 \\
41 & 27 & 03.5 \\
41 & 27 & 25.9 \\
41 & 27 & 56.7 \\
& & \\
41 & 28 & 08.6 \\
41 & 28 & 18.7 \\
41 & 28 & 28.1 \\
& & \\
41 & & \\
41 & 28 & 47.9 \\
41 & 29 & 18.9 \\
41 & 29 & 45.6\end{array}$ & $\begin{array}{lll}96 & 07 & 11.0 \\
96 & 07 & 02.1 \\
96 & 06 & 37.3 \\
96 & 06 & 02.8 \\
96 & 05 & 53.1 \\
96 & 05 & 53.0 \\
96 & 05 & 52.7 \\
96 & 05 & 52.7 \\
96 & 05 & 52.7 \\
96 & 05 & 52.8 \\
96 & 05 & 35.1 \\
96 & 05 & 13.4 \\
96 & 05 & 52.1 \\
& & \\
96 & 05 & 52.3 \\
96 & 05 & 52.4 \\
96 & 05 & 52.3 \\
& & \\
96 & 06 & 21.3 \\
96 & 06 & 25.5 \\
96 & 06 & 39.3\end{array}$ & $\begin{array}{r}5,136 \\
678 \\
1,890 \\
2,630 \\
739 \\
5,280 \\
2,630 \\
2,650 \\
5,240 \\
1,348 \\
2,802 \\
4,296\end{array}$ \\
\hline
\end{tabular}

Southeast along highways.

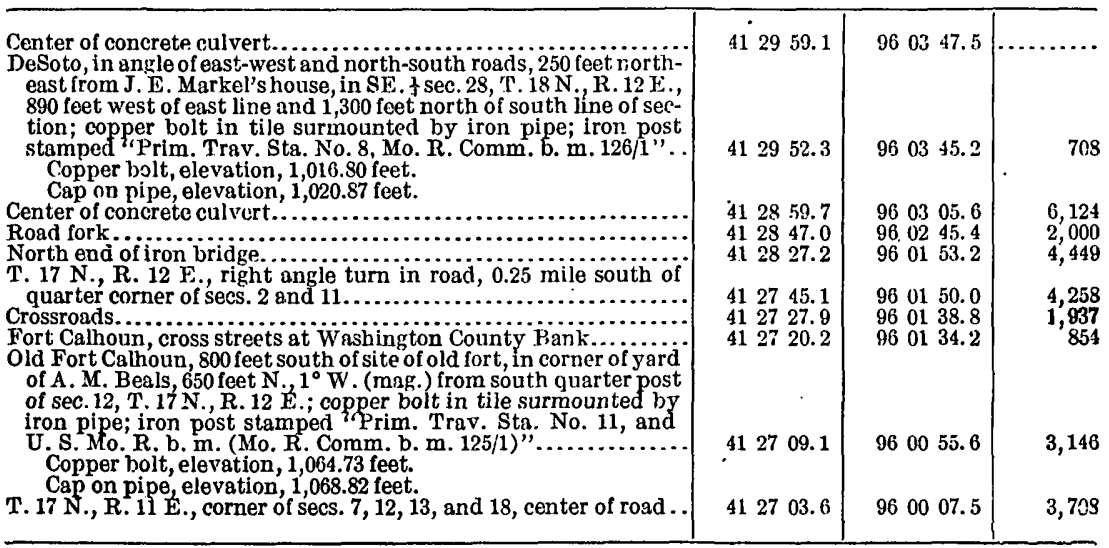


CALHOUN QUADRANGLE, NEBR.-Continued.

Northeast along Chicago \& Northwestern Ry.

\begin{tabular}{|c|c|c|c|}
\hline Station. & Latitude. & I.ongitude. & $\begin{array}{l}\text { Distance } \\
\text { between } \\
\text { stations. }\end{array}$ \\
\hline 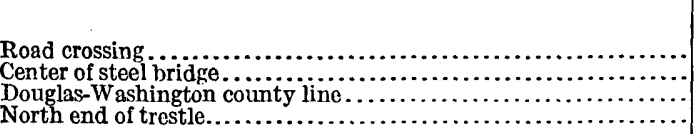 & $\begin{array}{lll}\circ & , & \prime \prime \\
41 & 22 & 31.4 \\
41 & 22 & 55.4 \\
41 & 23 & 34.8 \\
41 & 23 & 59.8\end{array}$ & $\begin{array}{lcc}\circ & \prime \prime & \prime \prime \\
96 . & 00 & 36.9 \\
96 & 00 & 26.2 \\
96 & 00 & 22.1 \\
96 & 00 & 03.9\end{array}$ & $\begin{array}{l}\text { Peet. } \\
2,569 \\
4,004 \\
2,882\end{array}$ \\
\hline
\end{tabular}

Magnetic declination for northeast part of quadrangle, $9.4^{\circ} \mathrm{E}$.; for southeast part of quadrangle, $8.8^{\circ} \mathrm{E}$.

\section{Along highways west through center of quadrangle.}

\begin{tabular}{|c|c|c|c|}
\hline 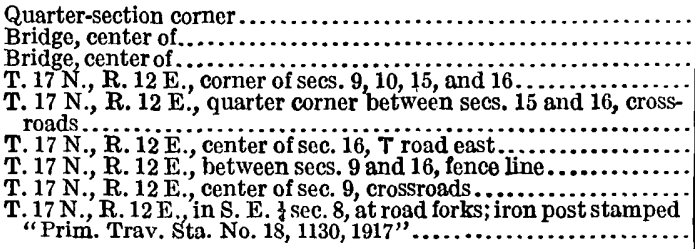 & $\begin{array}{lll}41 & 27 & 03.2 \\
41 & 27 & 03.8 \\
41 & 27 & 03.2 \\
41 & 27 & 03.6 \\
41 & 26 & 37.0 \\
41 & 26 & 37.3 \\
41 & 27 & 03.3 \\
41 & 27 & 25.9 \\
41 & 27 & 19.0\end{array}$ & $\begin{array}{lll}96 & 01 & 43.5 \\
96 & 02 & 41.0 \\
96 & 02 & 52.0 \\
96 & 03 & 33.6 \\
96 & 03 & 33.7 \\
96 & 04 & 08.8 \\
96 & 04 & 03.3 \\
96 & 04 & 08.2 \\
96 & 04 & 51.8\end{array}$ & $\begin{array}{r}4,381 \\
832 \\
3,169 \\
2,690 \\
2,670 \\
2,670 \\
2,320 \\
3,400\end{array}$ \\
\hline
\end{tabular}

Along highways west through south half of quadrangle.

Stone bridge, center of.

T. 17 N., Rs. 12 and 13 E., corner of secs. $25,30,31$ and 36 , crossroads

T. 17 N., R. 12 E., 1,386 feet east of corner of secs. $26,27,34$, and 35 ,

$T$. $17 \mathrm{~N}$., $R .12 \mathrm{E}$., on line through center of sec. 35 , center of road $\mathrm{T}$ at fence line............................................................ T. $17 \mathrm{~N}$, R. $12 \mathrm{E}$., quarter corner of sec. 2 , crossroads on DouglasT.17 N., R. 12 E., south corner of secs. 34 and 35 , fence line north

T. 17 N., R. 12 E., quarter corner, south side of sec. 34 , T road north

T. $16 \mathrm{~N}$., R. 12 E., quarter corner on north side of sec. $3, T$ road

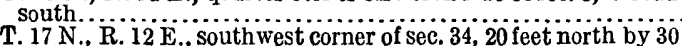
feet east of $T$ road north; (primary traverse station No. 15) iron

post stamped " Prim. Trav. Sta. No. $-, 1237,1917 \ldots \ldots \ldots \ldots \ldots$. T. 17 N., R. 12 E., corner of secs. 32 and 33 , T road north ........

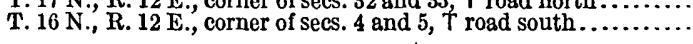

\begin{tabular}{|c|c|c|}
\hline $\begin{array}{lll}41 & 24 & 27.1 \\
41 & 24 & 27.2\end{array}$ & $\begin{array}{lll}96 & 00 & 46.0 \\
96 & 01 & 16.8\end{array}$ & 2,350 \\
\hline 412427.1 & 960207.6 & 3,879 \\
\hline 412401.1 & $96 \quad 0212.7$ & 2,664 \\
\hline $\begin{array}{lll}41 & 23 & 34.9 \\
41 & 23 & 34.8 \\
41 & 23 & 34.8\end{array}$ & $\begin{array}{lll}96 & 01 & 59.9 \\
96 & 02 & 23.4 \\
96 & 03 & 00.1\end{array}$ & $\begin{array}{l}2,827 \\
1,790 \\
2,800\end{array}$ \\
\hline $41 \quad 23 \quad 34.8$ & 960309.0 & 680 \\
\hline $\begin{array}{lll}41 & 23 & 35.2 \\
41 & 23 & 34.9 \\
41 & 23 & 34.9 \\
41 & 23 & 34.9\end{array}$ & $\begin{array}{lll}96 & 03 & 34.2 \\
96 & 04 & 09.3 \\
96 & 04 & 43.8 \\
96 & 04 & 52.8\end{array}$ & $\begin{array}{r}1,930 \\
2,670 \\
2,630 \\
690\end{array}$ \\
\hline
\end{tabular}

Along highways in east part of quadrangle.

T. 17 N., R. 12 E., 720 feet east by 30 feet north of corner of secs. 22 23,26 , and 27 , northeast corner of crossroads;iron post stamped (?) "Prim. Trav. Sta. No. 16, 1338, 1917".

\section{Road fork.}

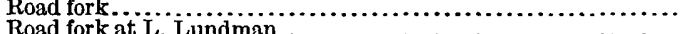

L. Lundman.

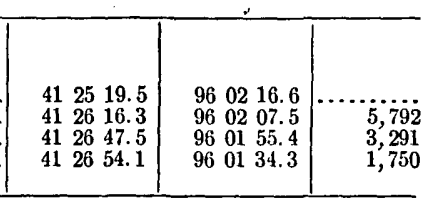

Magnetic declination for center of quadrangle, $9.6^{\circ} \mathrm{E}$.; for south part and east part of quadrangle, $9.5^{\circ} \mathrm{E}$.

\section{Southeast along highways in northeast corner of quadrangle. (By Oscar Jones in 1917.)}

\begin{tabular}{|c|c|c|c|}
\hline 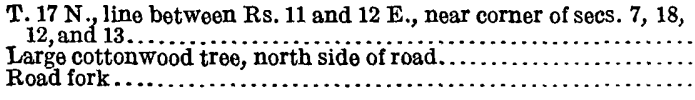 & $\begin{array}{lll}41 & 26 & 55.9 \\
41 & 26 & 55.0 \\
41 & 26 & 03.5\end{array}$ & $\begin{array}{lll}96 & 07 & 02.0 \\
96 & 06 & 46.0 \\
96 & 05 & 35.1\end{array}$ & $\begin{array}{r}1, \ddot{2} \ddot{19} \\
5,469\end{array}$ \\
\hline
\end{tabular}


Chalco QUAdRaNGLE, NEBR.

South along range line pear east border of quadrangle. (By Oscar Jones in 1917.)

\begin{tabular}{|c|c|c|c|}
\hline$\cdot \quad \cdots$ & Latitude. & Longitude. & $\begin{array}{l}\text { Distance } \\
\text { between } \\
\text { stations. }\end{array}$ \\
\hline 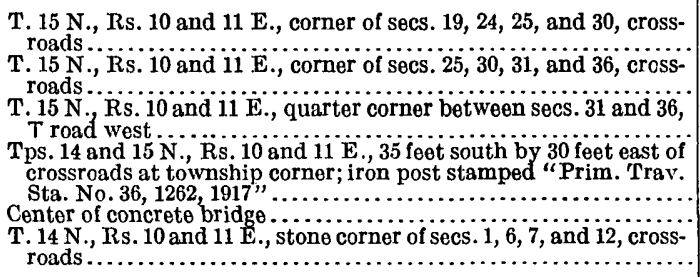 & $\begin{array}{ccc}0 & \prime & \prime \prime \\
41 & 14 & 53.2 \\
41 & 14 & 01.0 \\
41 & 13 & 34.9 \\
41 & 13 & 08.4 \\
41 & 12 & 42.6 \\
41 & 12 & 16.6\end{array}$ & $\begin{array}{lcc}0 & \prime \prime \prime \\
06 & 14 & 04.6 \\
96 & 14 & 04.4 \\
96 & 14 & 04.2 \\
96 & 14 & 03.8 \\
96 & 14 & 04.2 \\
96 & 14 & 04.1\end{array}$ & $\begin{array}{r}\text { Fect. } \\
\ldots \ldots \ldots \\
5,283 \\
2,649 \\
\\
2,676 \\
2,620 \\
2,624\end{array}$ \\
\hline
\end{tabular}

East along highways through center of quadrangle.

Center of wooden bridge

T.14 N., R. 11 E., corner of secs. $5,6,7$, and 8 , crossroads

T. 14 N., R. 11 E., corner of secs. $4,5,8$, and 9 , crossroads

T. 14 N., R. 11 E., crossroads, 40 feet south by 35 feet west of corner of secs. 3, 4, 9, and 10; iron post stamped "Prim. Trav. Sta.

No. 37, 1169, 1917 "

T. 14 N., R. 11 E., quarter corner botween secs. 3 and $10, T$ road

T. $14 \mathrm{~N} ., \mathrm{R} .11 \mathrm{E}$. , corner of secs. $2,3,10 \ldots \ldots \ldots \ldots \ldots \ldots$

T. 14 N., R. 11 E., corner of secs. $1,2,11$, and 12 , crossroads

T. 14 N., R. 11 is., quarter corner between secs. 1 and $12, \mathrm{~T}$ road

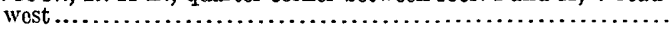

\begin{tabular}{llll|lll|l}
41 & 12 & 17.0 & 96 & 13 & 24.4 & \\
41 & 12 & 17.3 & 96 & 12 & 56.5 & 2,040 \\
41 & 12 & 17.8 & 96 & 11 & 47.8 & 5,130 \\
& & & & & \\
41 & 12 & 17.5 & 96 & 10 & 39.1 & \\
41 & 12 & 17.9 & 96 & 10 & 03.9 & 5,249 \\
41 & 12 & 18.3 & 96 & 09 & 29.4 & 2,685 \\
41 & 12 & 18.5 & 96 & 08 & 19.6 & 2,640 \\
& & & & & & \\
41 & 12 & 18.0 & 96 & 07 & 45.1 & & 2,638 \\
& & & & &
\end{tabular}

Magnetic declination through center of quadrangle, $9.6^{\circ} \mathrm{E}$.

South along highways near middle of quadrangle. (By Oscar Jones in 1917.)

T. 15 N., R .11 E., corner of secs. $21,22,27$, and 28 , crossroads

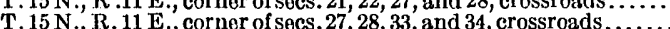

Tps. 14 and 15 N., R. 11 E., corner of secs. $3,4,33$, and 34 , cross-

T.14 N., R.1i E., Prim. Trav. Sta. No.37 (previously described)...

\begin{tabular}{lll|lll|r}
41 & 14 & 54.0 & 96 & 10 & 37.0 & $\ldots \ldots \ldots$ \\
41 & 14 & 01.5 & 96 & 10 & 36.9 & 5,305 \\
41 & 13 & 09.4 & 96 & 10 & 37.7 & \\
41 & 12 & 17.5 & 96 & 10 & 39.1 & 5,280 \\
& & & &
\end{tabular}

ELKHORN QUADRANGLE, NEBR.

North along highways. (By Oscar Jones in 1917.)

\begin{tabular}{|c|c|c|c|}
\hline Station. & Latitude. & Iongitude. & $\begin{array}{l}\text { Distance } \\
\text { between } \\
\text { stations. }\end{array}$ \\
\hline 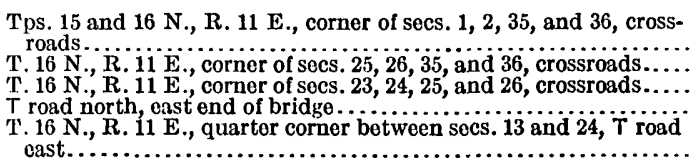 & $\begin{array}{ccc}0 & \prime & \prime \prime \\
41 & 18 & 23.2 \\
41 & 19 & 15.5 \\
41 & 20 & 07.8 \\
41 & 20 & 07.8 \\
41 & 21 & 00.2\end{array}$ & $\begin{array}{lcc}\circ & \prime \prime \\
96 & 08 & 19.2 \\
96 & 08 & 19.4 \\
96 & 08 & 19.9 \\
96 & 07 & 41.8 \\
96 & 07 & 44.8\end{array}$ & $\begin{array}{r}\text { Feet. } \\
\ldots . \\
5,288 \\
5,301 \\
2,902 \\
5,311\end{array}$ \\
\hline
\end{tabular}

South along highways near west border of quadrangle.

\begin{tabular}{|c|c|c|c|}
\hline 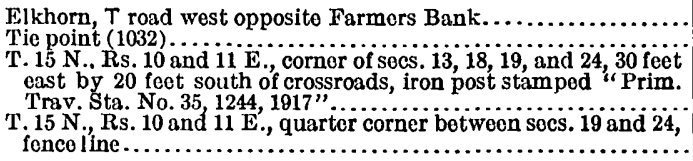 & $\begin{array}{lll}41 & 16 & 59.0 \\
41 & 16 & 52.0\end{array}$ & $\begin{array}{lll}96 & 14 & 13.2 \\
96 & 14 & 04.3 \\
96 & 14 & 04.1 \\
96 & 14 & 04.8\end{array}$ & 982 \\
\hline
\end{tabular}


ELKHORN QUADRANGLE, NEBR.-Continued.

East along highways near north border of quadrangle. (By Oscar Jones in 1917.)

\begin{tabular}{|c|c|c|c|}
\hline Station. & Latibude. & Longitude. & $\begin{array}{l}\text { Distance } \\
\text { between } \\
\text { stations. }\end{array}$ \\
\hline 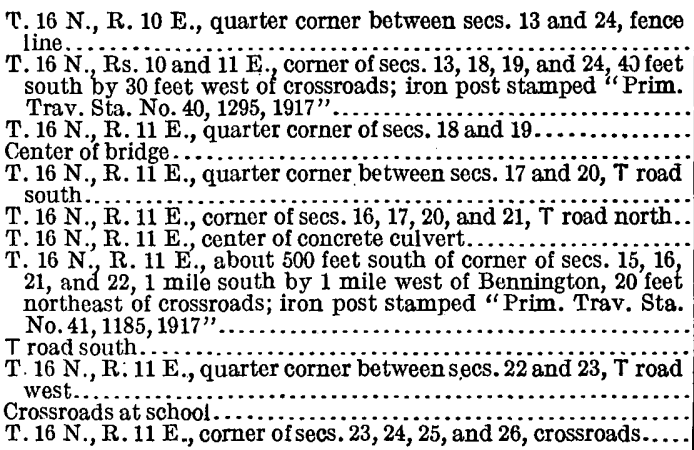 & $\begin{array}{ccc}\circ & \prime & \prime \prime \\
41 & 20 & 58.9 \\
& & \\
41 & 20 & 58.4 \\
41 & 20 & 58.9 \\
41 & 20 & 59.0 \\
41 & 20 & 59.1 \\
41 & 20 & 59.4 \\
41 & 20 & 59.6\end{array}$ & $\begin{array}{ccc}\circ & \prime & \prime \prime \\
96 & 14 & 38.4 \\
& & \\
96 & 14 & 03.9 \\
96 & 13 & 32.9 \\
96 & 13 & 09.7 \\
& & \\
96 & 12 & 23.4 \\
96 & 11 & 49.0 \\
96 & 11 & 14.9\end{array}$ & $\begin{array}{c}\text { Feet. } \\
\ldots . . . \\
\\
2,625 \\
2,363 \\
1,773 \\
3,526 \\
2,622 \\
2,597\end{array}$ \\
\hline
\end{tabular}

North along highways through center of quadrangle.

T. 16 N., R. 11 E., corner of secs. 16, 17, 20, and 21, T road north.

T. 16 N., R. 11 E., quarter corner between secs. 16 and 17 , fence

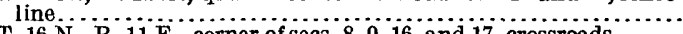

T. 16 N., R. 11 E., corner of secs, $8,9,16$ and 17 , crossroads

Center of wooden bridge.

\begin{tabular}{llll|lll|r}
41 & 20 & 59.4 & 96 & 11 & 49.0 & $\ldots \ldots$ & $\ldots$. \\
41 & 21 & 25.4 & 96 & 11 & 48.9 & \\
41 & 21 & 51.6 & 96 & 11 & 49.0 & 2,638 \\
41 & 22 & 15.1 & 96 & 11 & 49.1 & & 2,645 \\
& & & & & & \\
\hline
\end{tabular}

South along highways near center of quadrangle.

T. 16 N., R. 11 E., quarter corner between secs. 17 and 20, T road

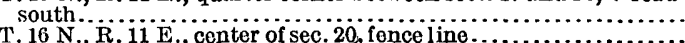

T. 16 N., R. $11 \mathrm{E}$, center of sec. 20 , fence line ...................

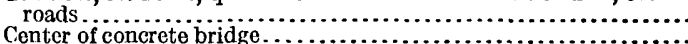

T. 16 N., R. 11 E., quarter corner between secs. 29 and 32 , $\mathrm{T}$ road

T. $16 \mathrm{~N} ., \mathrm{R} .11 \mathrm{E}$. corner of sees. $28,29,32$ and $33, \mathrm{~T}$ road south.

Tps. 15 and 16 N., R. 11 E., corner of secs. 4, 5, 32, and 33, crossroads .

\begin{tabular}{|c|c|}
\hline $\begin{array}{lll}41 & 20 & 59.1 \\
41 & 20 & 33.2\end{array}$ & $\begin{array}{llll}96 & 12 & 23.4 \\
96 & 12 & 23.2\end{array}$ \\
\hline $\begin{array}{lll}41 & 20 & 07.1 \\
41 & 19 & 45.5\end{array}$ & $\begin{array}{lll}96 & 12 & 23.0 \\
96 & 12 & 22.8\end{array}$ \\
\hline $\begin{array}{lll}41 & 19 & 14.9 \\
41 & 19 & 14.9 \\
41 & 18 & 22.7\end{array}$ & $\begin{array}{lll}96 & 12 & 22.5 \\
96 & 11 & 47.8 \\
96 & 11 & 47.1\end{array}$ \\
\hline
\end{tabular}

North and east along highways.

T. 15 N., Rs. 10 and 11 E., tie point.

T. 15 N., Rs. 10 and 11 E., corner of secs. $1,6,7$ and 12 , crossroads

Tps. 15 and 16 N., Rs. 10 and 11 E., 35 feet north by 30 feet east of crossroads, corner of secs. 1,6, 31, and 36 ; iron post stamped

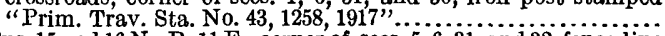

Tps. 15 and 16 N., R. $11 \mathrm{E}$. , corner of secs. $5,6,31$, and 32 , fence $\gtreqless$ line.

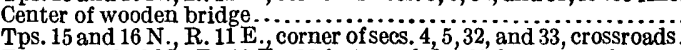

Tps. 15 and $16 \mathrm{~N} .$, R. $11 \mathrm{E}$. , corner of secs. $4,5,32$, and 33 , crossroads
Tps. 15 and $16 \mathrm{~N}$., R. 11 E., 30 feet north by 35 feet west of cross-

Tps. 15 and 16 N., R. 11 E., 30 feet north by 35 feet west of cross-
roads, corner of secs. $3,4,33$, and 34 ; iron post stamped "Prim.

Trav. Sta. No. 42, 1216, 1917',

Tps. 15 and 16 N., R. 11 E., corner of secs. $2,3,34$, and 35 , crossroads

Tps. 15 and 16 N., R. 11 E., corner of secs. 1, 2, 35, and 36, crossroads .

\begin{tabular}{|c|c|c|}
\hline $\begin{array}{lll}41 & 16 & 52.0 \\
41 & 17 & 30.0\end{array}$ & $\begin{array}{lll}96 & 14 & 04.3 \\
96 & 14 & 04.1\end{array}$ & 3,84 \\
\hline $\begin{array}{lll}41 & 18 & 22.6 \\
41 & 18 & 22.4 \\
41 & 18 & 22.6 \\
41 & 18 & 22.7\end{array}$ & $\begin{array}{lll}96 & 14 & 03.7 \\
96 & 12 & 56.4 \\
96 & 12 & 13.1 \\
96 & 11 & 47.1\end{array}$ & $\begin{array}{l}\mathbf{5}, \mathbf{3 2 3} \\
\mathbf{5}, \mathbf{1 3 0} \\
\mathbf{3}, \mathbf{3 0 0} \\
\mathbf{1}, \mathbf{9 8 4}\end{array}$ \\
\hline $\begin{array}{lll}41 & 18 & 23.1 \\
41 & 18 & 23.1 \\
41 & 18 & 23.2\end{array}$ & $\begin{array}{lll}96 & 10 & 38.4 \\
96 & 09 & 28.7 \\
96 & 08 & 19.2\end{array}$ & $\begin{array}{l}\mathbf{5}, 238 \\
\mathbf{5}, \mathbf{3 1 2} \\
\mathbf{5}, 300\end{array}$ \\
\hline
\end{tabular}

South along highways near center of quadrangle.

Prim. Trav. Sta. No. 42 (previously described)

T. 15 N., R. 11 E., corner of secs. $3,4,9$, and 10 , crossroads

T. 15 N., R. 11 E., corner of secs. $9,10,15$, and 16 , crossroads....

T. 15 N., R. 11 E., corner of secs. $15,16,21$, and 22,35 feet south by

30 feet east of crossroads; iron post stamped "Prim. Trav. Sta.

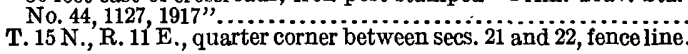

\begin{tabular}{|c|c|}
\hline $\begin{array}{lll}41 & 18 & 23.1 \\
41 & 17 & 30.7 \\
41 & 16 & 38.5\end{array}$ & $\begin{array}{lll}96 & 10 & 38.4 \\
96 & 10 & 38.6 \\
96 & 10 & 37.7\end{array}$ \\
\hline $\begin{array}{lll}41 & 15 & 45.8 \\
41 & 15 & 23.0\end{array}$ & $\begin{array}{lll}96 & 10 & 37.0 \\
96 & 10 & 37.2\end{array}$ \\
\hline
\end{tabular}

Magnetic declination for north border of quadrangle, $8.9^{\circ} \mathrm{E}$; ; for line through center of quadrangle, $8.4^{\circ} \mathrm{E}$; f for two lines near center of quadrangle, $9.2^{\circ} \mathrm{E}$.; for north and east borders of quadrangle, $9.1^{\circ} \mathrm{E}$. 
FLORENCE QUADRANGLE, NEBR.

West along highways. (By Oscar Jonee in 1911.)

\begin{tabular}{|c|c|c|c|}
\hline Station. & Latitude. & Longitude. & $\begin{array}{l}\text { Distance } \\
\text { between } \\
\text { stations. }\end{array}$ \\
\hline 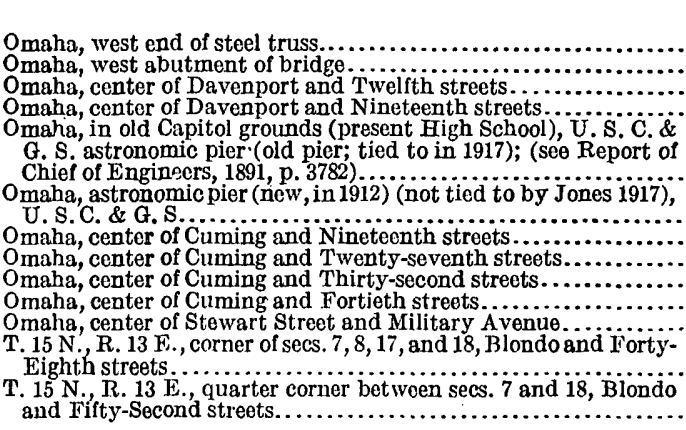 & $\begin{array}{ccc}\circ & \prime \prime \\
41 & 15 & 30.8 \\
41 & 15 & 30.7 \\
41 & 15 & 41.3 \\
41 & 15 & 41.6 \\
& & \\
41 & 15 & 35.94 \\
41 & 15 & 37.22 \\
41 & 16 & 04.3 \\
41 & 16 & 04.2 \\
41 & 16 & 04.2 \\
41 & 16 & 04.1 \\
41 & 16 & 26.4 \\
& & \\
41 & 16 & 39.8 \\
41 & 16 & 39.4\end{array}$ & \begin{tabular}{ccc}
$\circ$ & \multicolumn{1}{c}{$\prime \prime$} \\
95 & 55 & 22.9 \\
95 & 55 & 37.8 \\
95 & 55 & 54.2 \\
95 & 56 & 27.7 \\
& & \\
95 & 56 & 35.72 \\
& & \\
95 & 56 & 39.58 \\
95 & 56 & 27.6 \\
95 & 57 & 05.8 \\
95 & 57 & 36.8 \\
95 & 58 & 22.0 \\
95 & 58 & 40.8 \\
& & \\
95 & 59 & 07.1 \\
95 & 59 & 40.4
\end{tabular} & $\begin{array}{r}\text { Feet. } \\
1,139 \\
1,650 \\
2,553 \\
836\end{array}$ \\
\hline
\end{tabular}

Southeast along highways.

Center of concrete culvert, 100 feet south of road fork...................... Fence line east and west on section line between secs. 9 and $16, \bar{T}$. 16 N., R. 13 E.

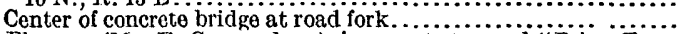

Florence (Mo. R. Comm. base); iron post stamped “Prim. Trav. Flarence Water Works, flagstaff on pump house (Mo. $\mathrm{R}$. Comm.

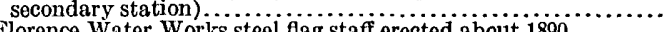

Florence Water Works steel tiag staff erected about 1890

\begin{tabular}{rrr|rrr|r}
41 & 22 & 25.4 & 95 & 57 & 12.0 & $\ldots \ldots \ldots$ \\
41 & 21 & 52.1 & 95 & 57 & 30.4 & \\
41 & 21 & 33.2 & 95 & 57 & 39.0 & 3,662 \\
41 & 20 & 34.4 & 95 & 57 & 30.6 & 2,033 \\
41 & 20 & 34.90 & 95 & 57 & 32.67 & 5,996 \\
41 & 20 & 35.0 & 95 & 57 & 30.5 & \\
\hline
\end{tabular}

Southeast along Chicago, St. Paul, Minneapolis \& Omaha Ry.

Road fork, 100 feet south of Florence station

Road crossing, railroad above road.

Road crossing corner between secs. 3 and 34 ; iron post Prim. Trav. Sta. No. 13 ,

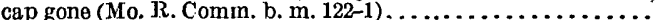

Ames Avenue, crossing of

Omaha, center of Loctist and Thirteenth streets, 0.25 mile east of

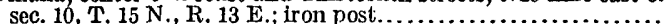
Omaha, center of $t$ ocust Street and Illinow Contrai $\mathrm{R}$. $\mathrm{R}$. crossing. Omaha, center of Locust and Twenty-Eighth streets............ Omaha, west abutment of Illinois Central R. R. bridge over wis-

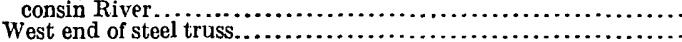

\begin{tabular}{|c|c|}
\hline $\begin{array}{lll}41 & 20 & 05.6 \\
41 . & 19 & 37.2 \\
41 & 19 & 23.2\end{array}$ & $\begin{array}{llll}95 & 57 & 20.6 \\
95 & 56 & 58.3 \\
95 & 56 & 42.0\end{array}$ \\
\hline $\begin{array}{lll}41 & 18 & 23.3 \\
41 & 17 & 58.3\end{array}$ & $\begin{array}{lll}95 & 56 & 13.4 \\
95 & 56 & 05.4\end{array}$ \\
\hline $\begin{array}{lll}41 & 17 & 06.1 \\
41 & 17 & 05.4 \\
41 & 17 & 05.3\end{array}$ & $\begin{array}{lll}95 & 55 & 56.9 \\
95 & 54 & 58.0 \\
95 & 53 & 42.4\end{array}$ \\
\hline $\begin{array}{lll}41 & 16 & 47.4 \\
41 & 16 & 44.1\end{array}$ & $\begin{array}{lll}95 & 53 & 36.5 \\
95 & 53 & 31.4\end{array}$ \\
\hline
\end{tabular}

West along Chicago \& Northwestern Ry. from Florence to Coffman.

West along highways.

Fort Omaha Reservation, near southeast gate of; iron post stamped

"Prim. Trav. Sta. No. 19 " ......................................... concrete pier.

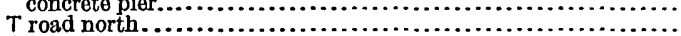

Crossroads.

$\begin{array}{lll}41 & 20 & 35.9 \\ 41 & 20 & 36.6 \\ 41 & 20 & 55.2\end{array}$

955823.3

$\begin{array}{lll}95 & 58 & 56.9\end{array}$

$95 \quad 5948.9$

\section{$\infty$}

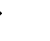


IRVINGTON QUADRANGLE, NEBR.

West along highways. (By Oscar Jones in 1917.)

\begin{tabular}{|c|c|c|c|}
\hline Station. & Latitude. & Longitude. & $\begin{array}{l}\text { Distance } \\
\text { between } \\
\text { stations. }\end{array}$ \\
\hline 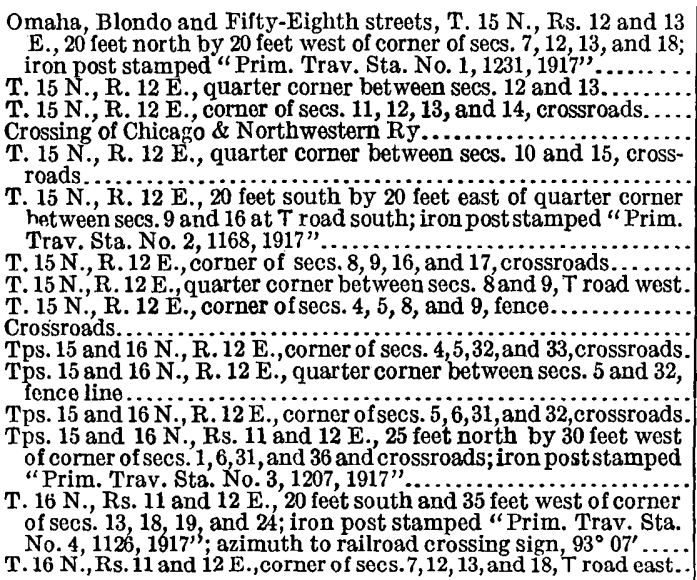 & 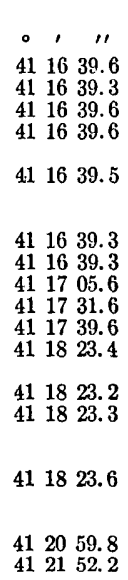 & $\begin{array}{llll}96 & 03 & 09.0 \\
& & \\
96 & 03 & 43.2 \\
96 & 04 & 52.5 \\
96 & 04 & 52.6 \\
96 & 04 & 52.6 \\
96 & 04 & 52.8 \\
96 & 04 & 52.7 \\
96 & 04 & 52.6 \\
& & \\
96 & 05 & 27.1 \\
96 & 06 & 01.9\end{array}$ & $\begin{array}{r}\mathbf{2 , 6 1 0} \\
\mathbf{5}, 290 \\
2,659 \\
\mathbf{2 , 6 3 0} \\
820 \\
4,427 \\
\mathbf{2 , 6 4 0} \\
2,650\end{array}$ \\
\hline
\end{tabular}

West along highways.

Tps. 15 and 16 N., Rs. 11 and 12 E., a quarter of a mile north of township corner; 20 fect south by 30 feet east of crossroads; iron post stamped "Prim. Trav. Sta. No, 20, 1251" ................

T.16 N., Rs. 11 and 12 E., 0.25 mile south of corner of secs. 25,30 ,

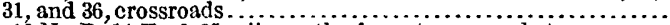
T. 16 N., R. 11 E., 0.25 mile south of quarter corner between secs. 25 and $36, T$ road north

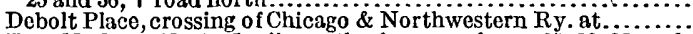

T. 16 N., R. 12 E., 0.25 mile north of corner of secs. $25,26,35$, and

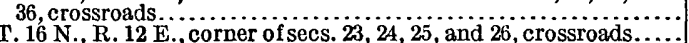
T. 16 N., R. 12 E., corner of secs. $23,24,25$, and 26 , crossroads..... T.16 N., R. 12 E., quartercorner between sees. 23 and 26, crossroads. T. 16 N., R. 12 E., corner of secs. $22,23,26$, and 27 , fence line.....

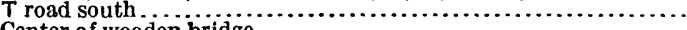
Center of wooden bridge.

T. 16 N., R. 12 E., corner of secs. $21,22,27$, and 28,30 feet north by 20 fect west of crossroads; iron post stamped "Prim. Trav. Sta. No. 21, 1233, 1917'

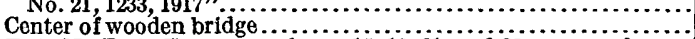
T. 16 N., R. 12 E., corner of secs. $15,16,21$, and 22 , crossroads..... Crossroads...

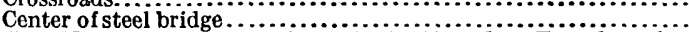

T. 16 N., R. 12 E., corner of secs. $17,18,19$, and 20 , T road south. Center of wooden bridge over Chicago \& Northwestern Ry

\begin{tabular}{|c|c|c|}
\hline $\begin{array}{lll}41 & 18 & 36.6\end{array}$ & $\begin{array}{lll}96 & 00 & 15.7\end{array}$ & \\
\hline $41 \quad 1903.0$ & 960016.2 & 2,675 \\
\hline $\begin{array}{lll}41 & 19 & 02.8 \\
41 & 19 & 27.8\end{array}$ & $\begin{array}{lll}96 & 00 & 50.7 \\
96 & 00 & 58.2\end{array}$ & $\begin{array}{l}2,646 \\
2,588\end{array}$ \\
\hline $\begin{array}{lll}41 & 19 & 29.0 \\
41 & 20 & 08.1 \\
41 & 20 & 08.1 \\
41 & 20 & 08.0 \\
41 & 20 & 08.0 \\
41 & 20 & 08.0 \\
41 & 20 & 08.0\end{array}$ & $\begin{array}{lll}96 & 01 & 25.3 \\
96 & 01 & 25.3 \\
96 & 01 & 42.4 \\
96 & 01 & 59.7 \\
96 & 02 & 34.1 \\
96 & 03 & 02.4 \\
96 & 03 & 10.6\end{array}$ & $\begin{array}{r}2,073 \\
3,960 \\
1,305 \\
1,325 \\
2,625 \\
2,165 \\
634\end{array}$ \\
\hline $\begin{array}{lll}41 & 20 & 08.3 \\
41 & 20 & 34.0 \\
41 & 21 & 00.2 \\
41 & 21 & 00.2 \\
41 & 21 & 00.2 \\
41 & 21 & 00.2 \\
41 & 21 & 00.2\end{array}$ & $\begin{array}{lll}96 & 03 & 43.4 \\
96 & 03 & 43.1 \\
96 & 03 & 43.2 \\
96 & 04 & 52.0 \\
96 & 05 & 23.8 \\
96 & 06 & 00.4 \\
96 & 06 & 29.7\end{array}$ & $\begin{array}{l}2,506 \\
2,599 \\
2,655 \\
5,257 \\
2,428 \\
2,790 \\
2,240\end{array}$ \\
\hline
\end{tabular}

West and north along Chicago \& Northwestern Ry.

Briggs, 315 feet west of , 40 feet south by 20 feet east of road crossing near southwest corner of sec. 18, T. 16 N., R. 13 E.; iron post stamped "Prim. Trav. Sta. No. 22, 1129"................... Road crossing. 
IRVINGTON QUADRANGLE, NEBR.-Continued.

South along highways.

\begin{tabular}{|c|c|c|c|}
\hline Station. & Jatitude. & Longitude. & $\begin{array}{l}\text { Distance } \\
\text { between } \\
\text { stations. }\end{array}$ \\
\hline 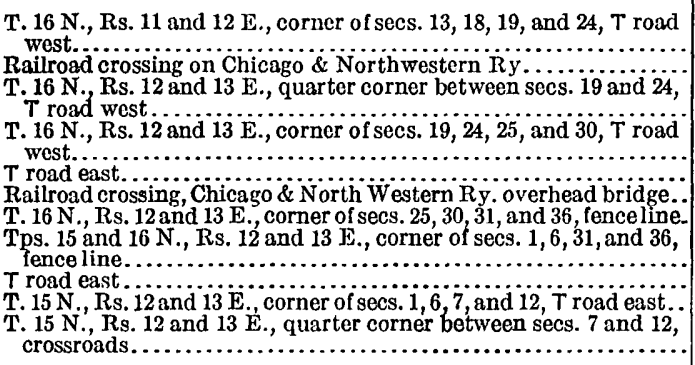 & $\begin{array}{lll}\circ & 1 & \prime \prime \\
41 & 21 & 00.3 \\
41 & 20 & 43.8 \\
41 & 20 & 34.1 \\
41 & & \\
41 & 20 & 08.1 \\
41 & 19 & 55.1 \\
41 & 19 & 18.0 \\
41 & 19 & 15.9 \\
41 & 18 & 23.9 \\
41 & 18 & 10.5 \\
41 & 17 & 31.7 \\
41 & 17 & 05.4\end{array}$ & \begin{tabular}{cccc}
$\circ$ & \multicolumn{1}{c}{} & $\prime \prime$ \\
96 & 00 & 16.6 \\
96 & 00 & 16.5 \\
96 & & \\
96 & 00 & 16.5 \\
96 & 00 & 16.4 \\
96 & 00 & 16.3 \\
96 & 00 & 16.2 \\
96 & 00 & 16.2 \\
96 & & & \\
96 & 00 & 16.3 \\
96 & 00 & 16.4 \\
96 & 00 & 16.6
\end{tabular} & $\begin{array}{r}\text { Feet. } \\
1,680 \\
980 \\
2,630 \\
1,320 \\
3,750 \\
220\end{array}$ \\
\hline
\end{tabular}

Magnetic declination for quadrangle, $9.8^{\circ} \mathrm{E}$.

North along highways near center of quadrangle.

\begin{tabular}{|c|c|c|c|}
\hline T. $15 \mathrm{~N} .$, R. $12 \mathrm{E}$, quarter corner between secs. 15 and 22 , cross- & 411547.3 & 960308.7 & \\
\hline $\begin{array}{l}\text { Center of bridge } \\
\text { T. } 15 \text { N., R. } 12 \text { E., quarter corner between secs. } 12 \text { and } 15 \text {, cross }\end{array}$ & 411619.0 & 960309.0 & 3,205 \\
\hline 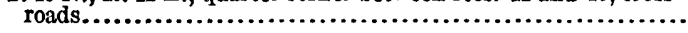 & 411639.5 & 960309.0 & 2,075 \\
\hline
\end{tabular}

South along highways near west border of quadrangle.

Tps. 15 and 16 N., Rs. 11 and 12 E., Prim. Trav. Sta. No. 3, pro viously described

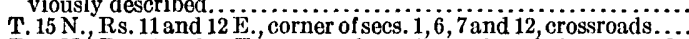
T. 15 N., Rs. 11 and 12 E., corner of secs. 7, 12, 13, and 18, crossroads. Center of steel bridge.

T. 15 N., Rs. 11 and $12 \mathrm{E} ., 30$ feet south by 40 feet east of crossroads at corner of secs. $13,18,19$, and 24 ; iron post stamped "Prim. Trav. Sta. No. 25, 1121, 1917"

\begin{tabular}{lll|lll|r}
41 & 18 & 23.6 & 96 & 07 & 10.5 & \\
41 & 17 & 31.1 & 96 & 07 & 10.2 & \\
41 & 16 & 38.8 & 96 & 07 & 10.2 & 5,313 \\
41 & 16 & 10.4 & 96 & 07 & 10.2 & 5,290 \\
& & & & & \\
41 & 15 & 46.3 & 96 & 07 & 09.8 & \\
& & & & & \\
& & &
\end{tabular}

North and east along highways. (By Oscar Jones in 1917.)

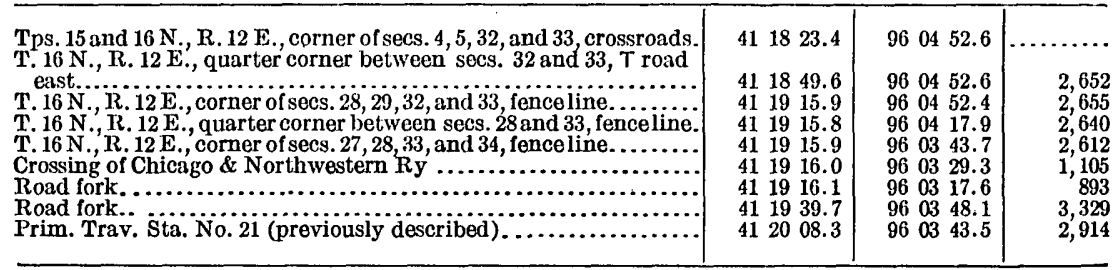

Magnetic declination north and east along highways, $9.2^{\circ} \mathrm{E}$. $121166^{\circ}-23-$ Bull. $709-16$ 
KENIARD QUADRANGLE, NEBR.

Southeast along highways. (By Oscar Jones in 1917.)

\begin{tabular}{|c|c|c|c|}
\hline Station. & Latitude. & Longitude. & $\begin{array}{l}\text { Distance } \\
\text { between } \\
\text { stations. }\end{array}$ \\
\hline 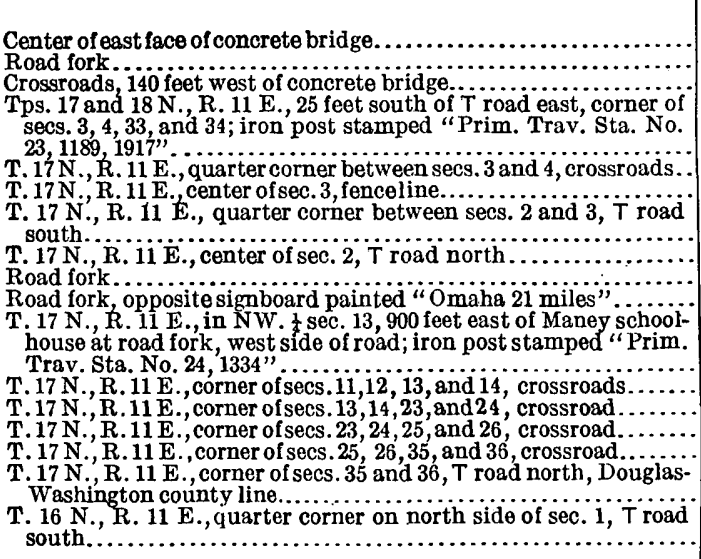 & $\begin{array}{lll}41 & 28 & 47.7 \\
41 & 28 & 22.0 \\
41 & 28 & 22.0 \\
41 & 28 & 22.1 \\
41 & 28 & 22.1 \\
41 & 27 & 55.8 \\
41 & 27 & 20.1 \\
& & \\
41 & 27 & 00.6 \\
41 & 27 & 04.0 \\
41 & 26 & 11.6 \\
41 & 25 & 19.6 \\
41 & 24 & 27.3 \\
41 & 23 & 35.0 \\
41 & 23 & 34.8\end{array}$ & $\begin{array}{lll}96 & 10 & 29.0 \\
96 & 10 & 29.6 \\
96 & 09 & 54.9 \\
& & \\
96 & 09 & 20.3 \\
96 & 08 & 45.5 \\
96 & 08 & 25.8 \\
96 & 08 & 11.2\end{array}$ & $\begin{array}{l}3,637 \\
2,604 \\
2,639 \\
\\
2,635 \\
2,645 \\
3,054 \\
3,777\end{array}$ \\
\hline
\end{tabular}

South along highways.

T. 17 N., R. 11 E., quarter corner between secs. 3 and 4 , crossroads.

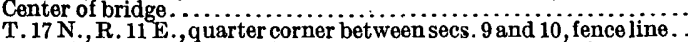
T. 17 N., R. 11 E., corner of secs. $9,10,15$, and 16 , crossroads Center of concrete bridge.

T. 17 N., R. 11 F., corner of sers. $15,16,21$, and 22,30 feet north by 20 feet west of crossroads, at Peaceful Hill schoolhouse; iron post stamped "Prim. Trav. Sta. No. 31, 1241, 1917"

T. $17 \mathrm{~N}$, R. 11 E., quarter corner between secs. 21 and 22, fence line T. 17 N R. $11 \mathrm{E}$., corner of secs. $21,22,27$, and 28 , crossroads........ T. 17 N., R. 11 E., quarter corner between secs. 22 and 27 , fence line T. 17 N.,R. 11 E., corner of secs. $27,28,33$, and 34 , crossroads. .

T. 17 N., R. 11 E., quarter corner between secs. 33 and 34 , f ence line

T. 16 N., R. 11 E., 50 feet west by 30 feet south of $T$ road south corner of secs. 3 and 4; iron post stamped "Prim. Trav. Sta. No. $32,1130 "$.

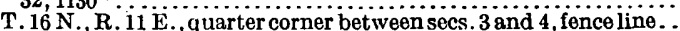

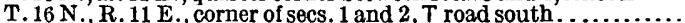

T. 17 N., R. 11 E., corner of secs. 35 and $36, T$ road north . ..........

\begin{tabular}{|c|c|c|}
\hline $\begin{array}{lll}41 & 28 & 22.0 \\
41 & 27 & 53.4 \\
41 & 27 & 29.8 \\
41 & 27 & 03.6 \\
41 & 26 & 41.2\end{array}$ & $\begin{array}{lll}96 & 10 & 29.6 \\
96 & 10 & 29.7 \\
96 & 10 & 29.8 \\
96 & 10 & 29.8 \\
96 & 10 & 29.5\end{array}$ & $\begin{array}{r}2,895 \\
2,390 \\
2,650 \\
2,276\end{array}$ \\
\hline $\begin{array}{lll}41 & 26 & 11.9 \\
41 & 25 & 45.4 \\
41 & 25 & 19.4 \\
41 & 24 & 53.2 \\
41 & 24 & 27.2 \\
41 & 24 & 01.0\end{array}$ & $\begin{array}{lll}96 & 10 & 30.4 \\
96 & 10 & 30.2 \\
96 & 10 & 29.8 \\
96 & 10 & 29.8 \\
96 & 10 & 30.0 \\
96 & 10 & 29.9\end{array}$ & $\begin{array}{l}2,966 \\
2,685 \\
2,631 \\
2,649 \\
2,635 \\
2,660\end{array}$ \\
\hline $\begin{array}{lll}41 & 23 & 34.6 \\
41 & 23 & 34.9 \\
41 & 23 & 34.8 \\
41 & 23 & 34.9\end{array}$ & $\begin{array}{lll}96 & 10 & 40.2 \\
96 & 09 & 55.3 \\
96 & 09 & 30.0 \\
95 & 09 & 20.4\end{array}$ & $\begin{array}{r}2,797 \\
3,423 \\
1,925 \\
732\end{array}$ \\
\hline
\end{tabular}

West and south along highways.

T. 17 N., R. 11 E., quarter corner between secs. 3 and $4 . . . \ldots \ldots .$. T. $17 \mathrm{~N}$., R. $11 \mathrm{E}$., in sec. 4 , T road south.

Center of steel bridge.

Kennard, on Chicago \& Northwestern $\mathbf{R y . , ~ o p p o s i t e ~ s e m a p h o r e , ~}$ center of track.

$T .17$ N., $R .11$ E., quarter corner between secs. 5 and $6, T$ road north T. 17 N., Rs. 10 and 11 E., quarter corner between sees. 1 and 6 , west side of road, at $T$ road east; iron post stamped "Prim.

Trav. Sta. No. 27, 1180, 1917'"

T. 17 N., Rs. 10 and $11 \mathrm{E}$, corner of secs. $1,6,7$, and 12 , crossroads.

T. 17 N., Rs. 10 and $11 \mathrm{E}$., quarter corner between secs. 7 and $12, T$ road east. .

T. 17 N., Rs. 10 and 11 E., quarter corner between secs. 13 and 18 ,

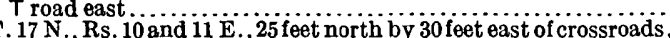
T. 17 N., Rs. 10 and 11 E., 25 feet north by 30 feet east of crossroads,
corner of secs. 13,18,19, and 24; iron post stamped "Prim. Trav. Sta. No. 26".

T. 17 N., Rs. 10 and 11 E., corner of secs. $19,24,25$, and 30 , T road T. 17 N., Rs. 10 and 11 E. quarter corner between secs. 25 and 30 ,

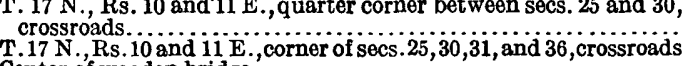
T. 17 N., Rs. 10 and $11 \mathrm{E}$. , corner of secs. 31 and 36 , T road north...

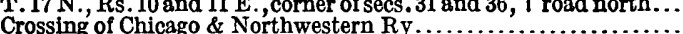
T. $16 \mathbf{N} ., \mathbf{R} .11 \mathrm{E} .$, corner of secs. 5 and $6, \mathrm{~T}$ road south $\ldots \ldots \ldots \ldots$ T. 17 N., R. 11 E., corner of secs. 31 and 32 , T road north .........

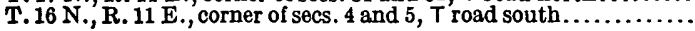

412822.0 412822.2

412822.4

412820.2

412822.2

412822.2

412756.2

412730.0

412637.8

412611.8

412519.3

412453.2

412427.1

4123.54 .5

412334.8

412334.8

412334.8

412334.7
961029.6

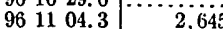

$961151.8 \quad 3,615$

961203.4 $96 \quad 1248.1$

927

3,400

961358.0

5,318 961357.6

2,640

$\begin{array}{lll}96 & 13 & 57.7\end{array}$

2,646

961358.0

$96 \quad 1357.6$

2,628

961358.0

5,318

961358.0 $96 \quad 1357.9$ $\begin{array}{lll}96 & 13 & 57.8\end{array}$

$\begin{array}{llll}96 & 13 & 57.9\end{array}$

$\begin{array}{llll}96 & 13 & 35.8\end{array}$

$\begin{array}{lll}96 & 12 & 58.7\end{array}$

961244.5

961149.1 
KENNARD QUADRANGLE, NEBR.-Continued.

East along highways.

\begin{tabular}{|c|c|c|c|}
\hline Station. & Latitude. & Longitude. & $\begin{array}{l}\text { Distance } \\
\text { between } \\
\text { stations. }\end{array}$ \\
\hline 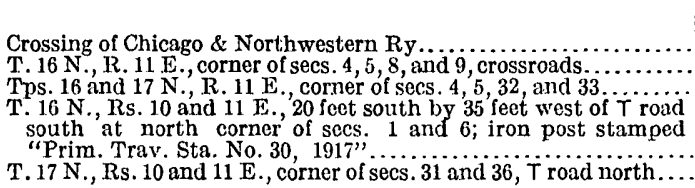 & $\begin{array}{ccc}\circ & \prime & \prime \prime \\
41 & 23 & 34.7 \\
41 & 22 & 43.9 \\
41 & 23 & 34.7 \\
& & \\
41 & 23 & 34.7 \\
41 & 23 & 34.9\end{array}$ & $\begin{array}{ccc}\circ & , & \prime \prime \\
96 & 14 & 22.9 \\
96 & 11 & 49.2 \\
96 & 11 & 49.1 \\
. & & \\
96 & 14 & 03.6 \\
96 & 13 & 57.9\end{array}$ & 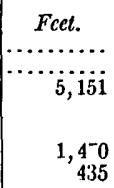 \\
\hline
\end{tabular}

Magnetic declination for the quadrangle, $8.8^{\circ} \mathrm{E}$.

RALSTON QUADRANGLE, NEBR.

North near west border and east near north border of quadrangle.

\begin{tabular}{|c|c|c|c|}
\hline Station. & Latitude. & Longitude. & $\begin{array}{l}\text { Distance } \\
\text { between } \\
\text { stations. }\end{array}$ \\
\hline 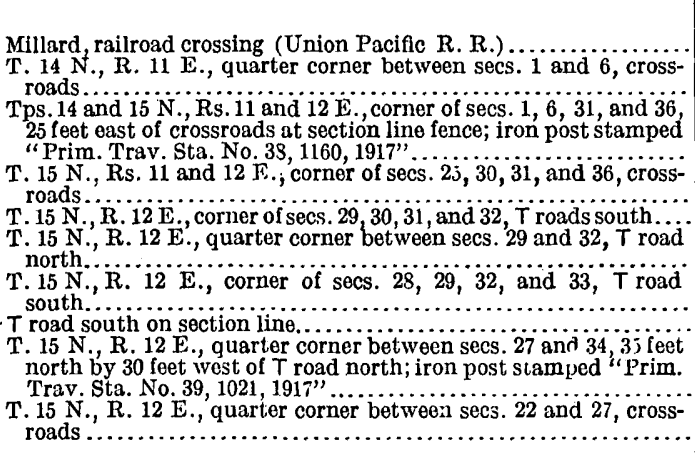 & $\begin{array}{ccc}\circ & \prime & \prime \prime \\
41 & 12 & 35.1 \\
41 & 12 & 43.7 \\
& & \\
41 & 13 & 09.8 \\
41 & 14 & 02.1 \\
41 & 14 & 02.2 \\
41 & 14 & 02.3 \\
41 & 14 & 02.4 \\
41 & 13 & 59.5\end{array}$ & $\begin{array}{ccc}\circ & \prime & \prime \prime \\
96 & 07 & 25.5 \\
96 & 07 & 10.1 \\
& & \\
96 & 07 & 09.9 \\
96 & 01 & 10.2 \\
96 & 06 & 02.4 \\
96 & 05 & 27.8 \\
96 & 04 & 53.1 \\
96 & 03 & 43.7\end{array}$ & \begin{tabular}{|r|} 
Feet. \\
1,463 \\
2,647 \\
5,285 \\
5,175 \\
2,645 \\
2,652 \\
5,302 \\
2,639 \\
5,273
\end{tabular} \\
\hline
\end{tabular}

\section{South near west border of quadrangle.}

T. 15 N., Rs. 11 and $12 \mathrm{E}$., corner of secs. $19,24,25$, and 30 , crossT. $15 \mathrm{~N} ., \mathrm{Rs} .11$ and $12 \mathrm{E}$. , corner of secs. $25,30,31$, and $33 \ldots \ldots \ldots$.

Magnetic declination for north edge of quadrangle, $9.3^{\circ} \mathrm{E}$. 
WATERIOO QUADRANGLE, NEBR.

South and east along highways near east border of quadrangle. (By Oscar Jones in 1917.)

\begin{tabular}{|c|c|c|c|}
\hline Station. & Latitude. & Longitude. & $\begin{array}{l}\text { Distance } \\
\text { between } \\
\text { stations. }\end{array}$ \\
\hline 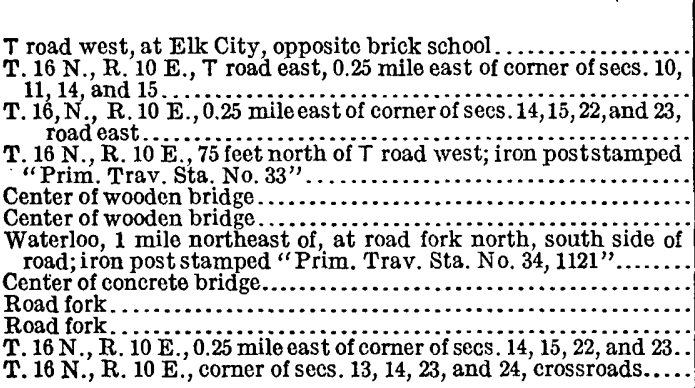 & $\begin{array}{ccc}\circ & \prime & \prime \prime \\
41 & 22 & 17.3 \\
41 & 21 & 51.2 \\
& & \\
41 & 20 & 58.9 \\
& & \\
41 & 20 & 07.5 \\
41 & 19 & 11.0 \\
41 & 18 & 26.4 \\
& & \\
41 & 17 & 40.5 \\
41 & 17 & 40.9 \\
41 & 17 & 34.9 \\
41 & 17 & 30.3 \\
41 & 20 & 58.9 \\
41 & 20 & 59.0\end{array}$ & $\begin{array}{ccc}\circ & \prime & \prime \prime \\
96 & 16 & 04.8 \\
& & \\
96 & 16 & 04.8 \\
& & \\
96 & 16 & 05.0 \\
& & \\
96 & 16 & 31.0 \\
96 & 16 & 26.7 \\
96 & 16 & 24.0 \\
& & \\
96 & 16 & 37.0 \\
96 & 15 & 51.5 \\
96 & 15 & 14.1 \\
96 & 15 & 00.0 \\
96 & 16 & 05.0 \\
96 & 15 & 13.5\end{array}$ & \begin{tabular}{|r|} 
Feet. \\
2,640 \\
5,292 \\
\\
5,575 \\
5,730 \\
4,513 \\
4,757 \\
3,465 \\
2,922 \\
1,172 \\
3,930
\end{tabular} \\
\hline
\end{tabular}

Magnetic declination for east border of quadrangle, $9.4^{\circ} \mathrm{E}$.

FLORENCE QUADRANGLE, IOWA.

West along highways. (By Oscar Jones in 1917.)

\begin{tabular}{|c|c|c|c|}
\hline Station. & Latitude. & Longitude. & $\begin{array}{l}\text { Distance } \\
\text { between } \\
\text { stations. }\end{array}$ \\
\hline 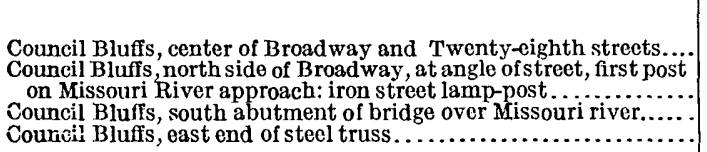 & $\begin{array}{ccc}\circ & \prime & \prime \prime \\
41 & 15 & 42.7 \\
41 & & \\
41 & 15 & 42.6 \\
41 & 15 & 33.2 \\
41 & 15 & 31.5\end{array}$ & $\begin{array}{ccc}\circ & \prime & \prime \prime \\
95 & 53 & 20.3 \\
& & \\
95 & 54 & 34.9 \\
95 & 55 & 03.2 \\
95 & 55 & 17.8\end{array}$ & $\begin{array}{r}\text { Fect. } \\
\text {. } \\
5,697 \\
2,362 \\
1,125\end{array}$ \\
\hline
\end{tabular}

\section{At Council Bluffs.}

Council Bluffs, east abutment of Illinois Central R. R. bridge..... Council Bluffs, road crossing.

\begin{tabular}{|c|c|}
\hline $\begin{array}{lll}41 & 16 & 35.2 \\
41 & 16 & 32.2\end{array}$ & $\begin{array}{lll}95 & 53 & 21.8 \\
95 & 52 & 45.7\end{array}$ \\
\hline
\end{tabular}

HONEY CREEK QUADRANGLE, IOWA.

In Council Bluffs on Illinois Central R. R. (By Oscar Jones in 1917.)

\begin{tabular}{|c|c|c|c|}
\hline Station. & Latitude. & Longitude. & $\begin{array}{l}\text { Distance } \\
\text { between } \\
\text { stations }\end{array}$ \\
\hline 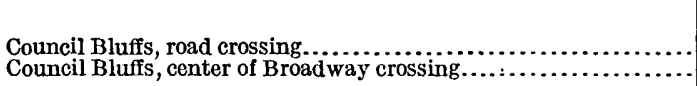 & $\begin{array}{ccc}\circ & \prime & \prime \prime \\
41 & 16 & 04.5 \\
41 & 15 & 42.5\end{array}$ & $\begin{array}{ccc}\circ & \prime & \prime \prime \\
95 & 52 & 14.6 \\
95 & 52 & 14.0\end{array}$ & $\begin{array}{c}\text { Feet. } \\
\cdots 2,235\end{array}$ \\
\hline
\end{tabular}

Magnetic declination for Council Bluffs, about $9^{\circ} \mathrm{E}$.

In Council Bluffs.

\begin{tabular}{|c|c|c|c|}
\hline $\begin{array}{l}\text { Council Bluffs, center of Sixteenth Street and Fifth Avenue } . . . . \\
\text { Council Bluffs, center of Sixteenth Street and Broadway........... }\end{array}$ & $\begin{array}{lll}41 & 15 & 25.1 \\
41 & 15 & 42.3\end{array}$ & $\begin{array}{lll}95 & 52 & 02.8 \\
95 & 52 & 02.8\end{array}$ & 1,749 \\
\hline
\end{tabular}


OMAHA AND VICINITY QUADRANGLE, IOWA.

Along streets in Council Bluffs. (By Oscar Jones in 1917.)

\begin{tabular}{|c|c|c|c|}
\hline Station. & Latitude: & Longitude. & $\begin{array}{l}\text { Distance } \\
\text { between } \\
\text { stations. }\end{array}$ \\
\hline 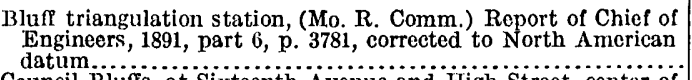 & $\begin{array}{ccc}\circ & & \prime \prime \\
41 & 14 & 34.44\end{array}$ & 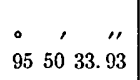 & Feet. \\
\hline $\begin{array}{l}\text { Council Bluffs, at Sixteenth Avenue and High Street, center of } \\
\text { sewer manhole. } \\
\text { Council Bluffs, at Sixteenth A venue and } \text { Tenth Street, at turn of }\end{array}$ & $\begin{array}{lll}41 & 14 & 47.4\end{array}$ & $95 \quad 5047.8$ & 1,683 \\
\hline 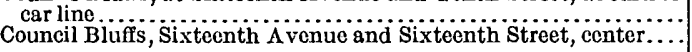 & 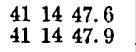 & $\begin{array}{lll}95 & 51 & 31.9 \\
95 & 52 & 03.2\end{array}$ & $\begin{array}{l}3,365 \\
2,389\end{array}$ \\
\hline
\end{tabular}

Magnetic declination for Council Bluffs, about $9^{\circ} \mathrm{E}$.

\section{OKLAHOMA.}

\section{PRIMARY TRAVERSE.}

Agra, Belford, Claremore, Foraker, Gray Horse, Hominy, Kaw, Mallon, Newkirk, Nowata, Pawhuska, Prague, Sac and Fox, Skedee, and Yale quadrangles.

Creek, Kay, osage, Payne, hincoln, okfuskee, PaWnee, and tulsa counties.

The following geographic positions, on North American datum, were determined by primary traverse.in 1916 by B. H. Yoakum and W. F. Hicks and are based on U. S. Geological Survey triangulation stations, Cox, Cushing, Flint, and Phanton, and primary traverse positions determined in 1914 .

The work in most of Claremore, Pawhuska and the northern part of Hominy quadrangles was done by Hicks; that in the other quadrangles named above and in part of Hominy quadrangle was done by Yoakum.

For other positions in Oklahoma see U. S. Geological Survey Bulletins 122, 175, 310, 440, and 644, and U. S. Coast and Geodetic Survey Report for 1903.

\section{AGRA QUADRANGLE.}

Along highways in the northeast corner of quadrangle. (By B. H. Yoakum in 1916.)

\begin{tabular}{|c|c|c|c|}
\hline Station. & Latitude. & Iongitude. & $\begin{array}{l}\text { Distance } \\
\text { between } \\
\text { stations. }\end{array}$ \\
\hline 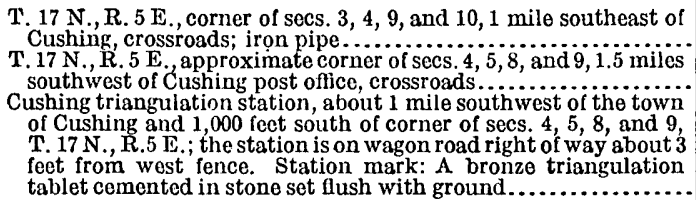 & $\begin{array}{ccc}0 & \prime & \prime \prime \\
35 & 58 & 16.0 \\
35 & 58 & 16.0\end{array}$ & $\begin{array}{ccc}\circ & \prime & \prime \prime \\
96 & 46 & 02.7 \\
96 & 47 & 05.4\end{array}$ & $\begin{array}{l}\text { Feet. } \\
5,363 \\
5,149\end{array}$ \\
\hline
\end{tabular}

Magnetic declination for the extreme northeast corner of quadrangle, $9^{\circ} 18^{\prime} \mathrm{E}$. 
BELFORD QUADRATTLE.

South along highways near west border of quadrangle. (By B. H. Yoakum in 1916.)

\begin{tabular}{|c|c|c|c|}
\hline Station. & Latitude. & Longitude. & $\begin{array}{l}\text { Distance } \\
\text { between } \\
\text { stations. }\end{array}$ \\
\hline 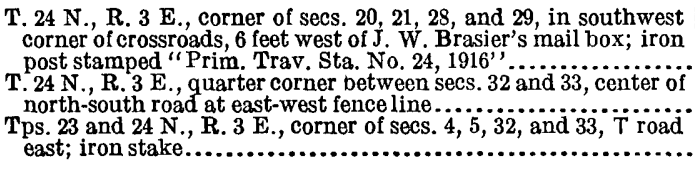 & $\begin{array}{ccc}\circ & \prime & \prime \prime \\
36 & 32 & 08.1 \\
36 & 30 & 51.5 \\
36 & 30 & 25.4\end{array}$ & $\begin{array}{ccc}\circ & \prime & \prime \prime \\
96 & 59 & 47.7 \\
96 & 59 & 47.4 \\
96 & 59 & 45.9\end{array}$ & $\begin{array}{l}\text { Feet. } \\
7,740 \\
2,643\end{array}$ \\
\hline
\end{tabular}

Magnetic declination for west border of quadrangle, $10^{\circ} 30^{\prime} \mathrm{E}$.

East along highways near south border of quadrangle.

Tps. 23 and 24 N., R. 3 E., quarter corner between secs. 4, and 33, at north and south fence line; iron stake..................

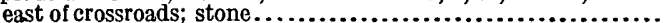

Tps. 23 and 24 N., $R .3$ E., corners of secs. $2,3,34, \ldots$ and 35,7 road

rps: 23 and 24 N., R. 3 E., quarter corner between secs. 2 and 35

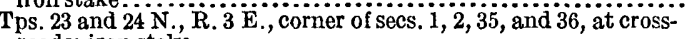
roads; iron stake................................................

Tps. 23 and 24 N., R. 3 E., quarter corner between secs. 1 and 36 ,

Tps. 23 and 24 N., Rs. 3 and 4 E., corner of secs. $1,6,31$, and 36 , center of east-west road, 20 feet east of fence line south; Gen-

Tps. 23 and 24 N., $R .4$ E., corner of secs. $5,6,31$, and $32, \ldots \ldots \ldots$ and south fence line; General Land Officeiron post with cap missing.

Tps. 23 and 24 N., R. 4 E., quarter corner between secs. .5 , and 32 center of east-west road in line with road south; General

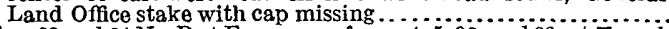

Tps. 23 and 24 N, R. 4 E., corner of secs. $4,3,32$, and 33 , at $T$ road south; General Land Óffice stake with cap missing.

Tps. 23 and 24 N., R. 4 E., quarter corner between secs. 4 and 33 , at T road north; General land Office stake with cap missing.

T. 24 N., R. 4 E., corner of secs. 3, 4, 33, and 34, in northeast corner of crossroads at Belford schoolhouse, district 42 ; iron post stamped "Prim. Trav. Sta. No. 25, 1916."

Tps. 23 and 24 N., R. 4 E., quarter corner between secs. 3 and $34 \ldots$

Tps. 23 and 24 N., R. 4 E., corner of secs. $2,3,34$, and $35,0.4$ mile west of Belford, center of east-west road at $T$ road north..........

Center of east end of bridge over the east pier of wagon bridge over Arkansas River, about 1.2 miles east of Belford, formerly Belford, $\mathrm{P} . \mathrm{O}$

Tps. 23 and 24 N., R. 2 E., corner of secs. $1,2,35$, and 36 , at cross-

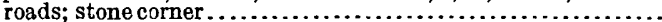

Tps. 23 and 24 N., R. 4 E., quarter corner between secs. 1 and 36 ,

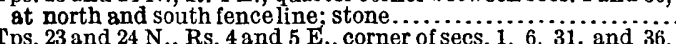
Tps. 23 and 24 N., Rs. 4 and 5 E. , corner of secs. $1,6,31$, and 36 ,

Tps. 23 and 24 N., R., 5 E., corner of secs. $5,6,3 i$, and 32 , at cross-

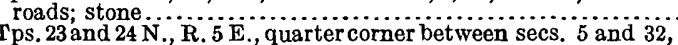

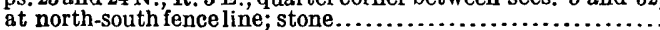

Tps. 23 and 24 N., R. 5 E., approximate corner of secs. $4,5,32$, and 33 , center of east and west road at $T$ road south, 75 feet east of $T$

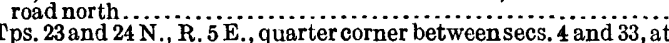
north and south fence line....................................... Tps. 23 and 24 N., R. 5 E., corner of secs. $3,4,33$, and 34 , at cross-

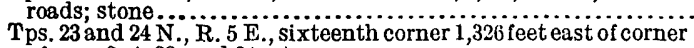

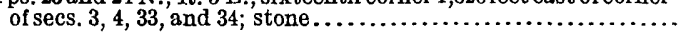

363025.5

363025.5

363025.4

363025.6

363025.7

363025.6

363025.6

$\begin{array}{lll}36 & 30 & 25.7\end{array}$

$\begin{array}{lll}36 & 30 & 25.7\end{array}$

363025.8

$\begin{array}{lll}36 & 30 & 25: 9\end{array}$

$\begin{array}{lll}36 & 30 & 26.1\end{array}$

363025.9

363025.9

363026.0

363026.0

363026.0

363026.2

$36 \quad 3025.9$

$\begin{array}{lll}36 & 30 & 25.8\end{array}$

363025.6

363025.5

$\begin{array}{lll}36 & 30 & 25.4\end{array}$

363025.4
965915.0

$96 \quad 58 \quad 42.7$

965738.1

$\begin{array}{lll}96 & 57 & 05.7\end{array}$

965633.4

965601.0

$\begin{array}{lll}96 & 5528.7\end{array}$

965423.9

965351.7

$\begin{array}{lll}96 & 53 & 19.3\end{array}$

$\begin{array}{lll}96 & 52 & 33.8\end{array}$

$9652 \quad 14.5$

965142.6

$9651 \quad 10.4$

965027.7

$96 \quad 50 \quad 05.9$

964933.6

964901.2

964758.5

964726.3

964654.1

964621.9

$\begin{array}{lll}96 & 45 & 49.7\end{array}$

964533.5
2, 525

2,635

5,275

2,640

2,640

2,640

2,640

5,289

2,630

2, 640

3,717

1,578

2, 600

2, 626

3,490

1, 777

2,640

2, 642

5,124

2,629

2,627

2,624

2,629

1,326

Magnetic declination for south border of quadrangle, $10^{\circ} 01^{\prime} \mathrm{E}$. 
BELFORD QUADRANGIE-Continued.

West along highways through center of quadrangle.

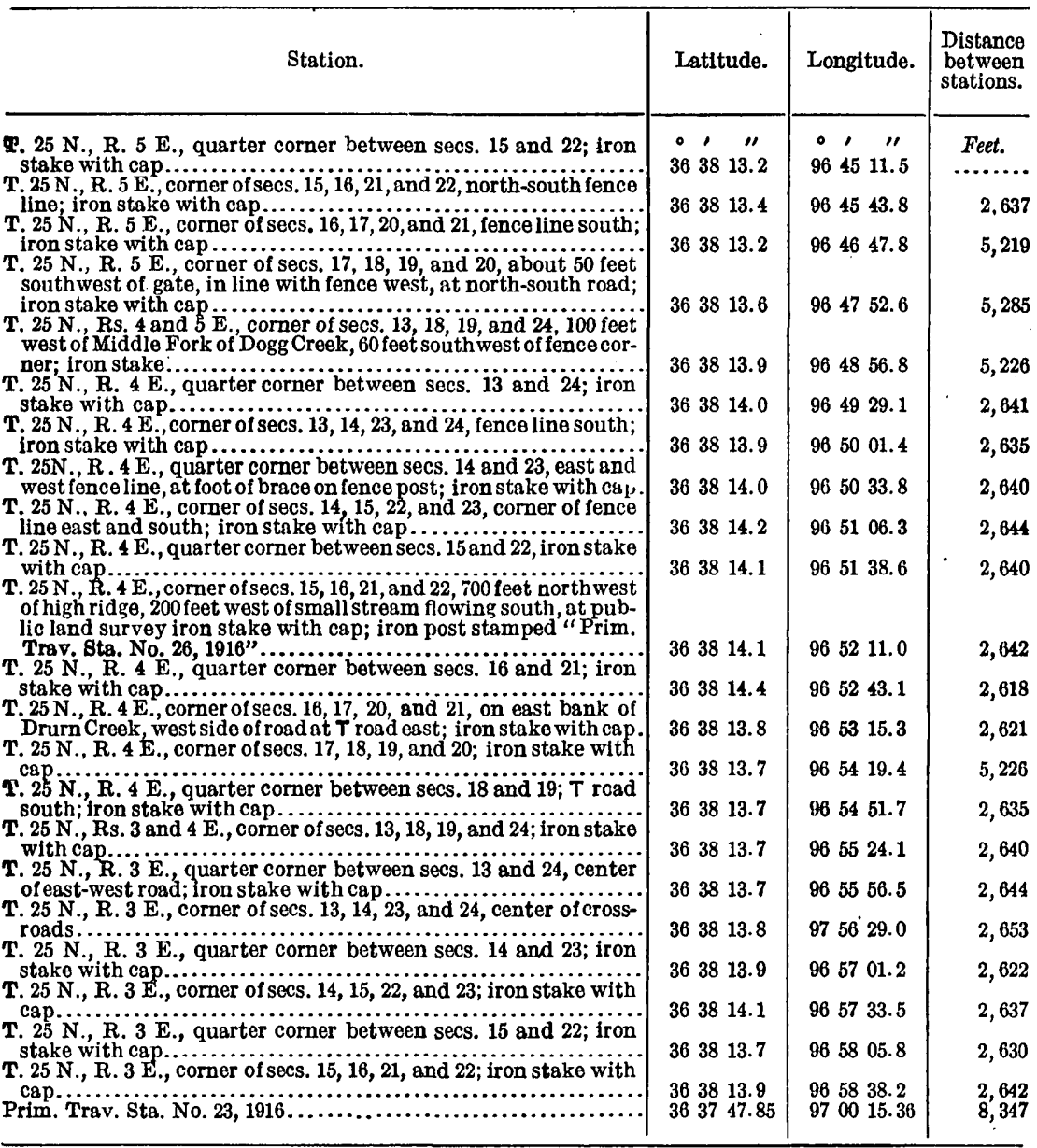

Magnetic declination through center of quadrangle (east-west), $10^{\circ} 13^{\prime} \mathrm{E}$.

Along highweys and Atchison, Topeka \& Santa Fe Ry.

\begin{tabular}{|c|c|c|c|}
\hline 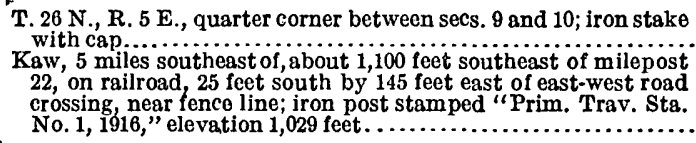 & $\begin{array}{l}3644 \quad 44.2 \\
36 \quad 44 \quad 17.9\end{array}$ & $96 \quad 45 \quad 41.4$ & 3,020 \\
\hline
\end{tabular}


CLAREMORE QUADRATGLE.

North along public roads near southwest corner of quadrangle. (By B. H. Yoakum in 1916.)

\begin{tabular}{|c|c|c|c|}
\hline Station. & Iatitude. & Iongitude. & $\begin{array}{l}\text { Distance } \\
\text { between } \\
\text { stations. }\end{array}$ \\
\hline 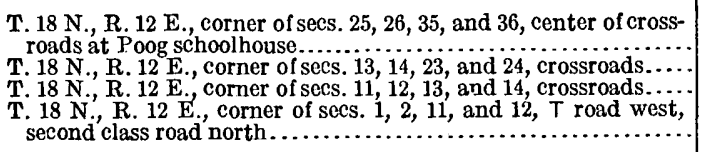 & $\begin{array}{ccc}\circ & \prime & \prime \prime \\
36 & 00 & 10.6 \\
36 & 01 & 55.1 \\
36 & 02 & 47.6 \\
36 & 03 & 39.8\end{array}$ & $\begin{array}{ccc}\circ & \prime & \prime \prime \\
95 & 59 & 36.7 \\
95 & 59 & 36.5 \\
95 & 59 & 36.5 \\
95 & & \\
99 & 36.5\end{array}$ & $\begin{array}{r}\text { Feet. } \\
10,570 \\
5,305 \\
5,280\end{array}$ \\
\hline
\end{tabular}

From Tulsa north via highways and Midland Valley R. R. to Sperry.

NoTE.-An error of about $2.4^{\prime \prime}$ in latitude and $1.6^{\prime \prime}$ in longitude exists between Tulsa and Sperry.

Tulsa, 323 feet north of intersection of "Frisco" Ry. and First Street, south side of First Street: top of curbing painted " 675 ". T. 20 N., R. 12 E., corner of secs. $25,26,35$, and 36 , crossroads....
360910.7 361037.0
955954.1 $95 \quad 5935.8$

Along public roads near southwest corner of quadrangle.

T. 20 N., R. 12 E., 0.3 mile west of northeast corner of sec. 24 , east side of Midland Valley R. R. and south side of wagon road; iron post stamped "Prim. Trav. Sta. No. 48, 1916"................ T. 20 N., Rs. 12 and 13 E., 0.25 mile west of corner of secs. 1, 6, 7,

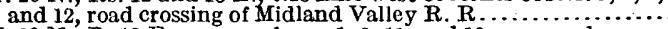
T. 20 N., R. 12 E., corner of secs. 1, 2, 11, and 12, crossroads....... T. 20 N., Rs. 12 and 13 E., north corner of secs. 1 and 6 ; iron post stamped "Prim. Trav. Sta. No. 50, 1916. Elev. 627".

T. 21 N., R. 12 E., 0.25 mile west of northeast corner of sec. 36 , at railroad crossing on section line, on east side of railroad and south side of wagon road; iron post stamped "Prim. Trav. Sta. No. 51, 1916. Elev. 621 Okla."..............................

\begin{tabular}{|c|c|c|}
\hline 361221.4 & $95 \quad 58 \quad 45.2$ & 11,346 \\
\hline $\begin{array}{lll}36 & 14 & 03.4 \\
36 & 14 & 03.2\end{array}$ & $\begin{array}{lll}95 & 58 & 46.7 \\
95 & 59 & 34.4\end{array}$ & $\begin{array}{r}10,315 \\
3,910\end{array}$ \\
\hline 361455.6 & $95 \quad 58 \quad 29.7$ & \\
\hline $\begin{array}{llll}36 & 15 & 47.6\end{array}$ & $\begin{array}{llll}95 & 58 & 34.1\end{array}$ & 5,262 \\
\hline
\end{tabular}

5,262

Along public roads near southwest corner of quadrangle.

The following adjusted positions are on a circuit which closes satisfactorily.

\begin{tabular}{|c|c|c|c|}
\hline $\begin{array}{l}\text { Sperry, } 1.25 \text { miles southeast of, road crossing of Midland Valley R.R. } \\
\text { T. } 21 \text { N., R. } 12 \text { E., corner of secs. } 23,24,25 \text {, and } 26 \text {, T road north.. }\end{array}$ & $\begin{array}{lll}36 & 16 & 42.5 \\
36 & 16 & 42.4\end{array}$ & $\begin{array}{lll}95 & 59 & 00.1 \\
95 & 59 & 20.7\end{array}$ & $\begin{array}{l}5,666 \\
1,690\end{array}$ \\
\hline
\end{tabular}

Position by F. W. Hicks.

\begin{tabular}{l|r|r|r|r}
\hline Sperry, Main Street crossing of Midland Valley R. R............. & 361747.5 & 955929.1 & 6,986 \\
\hline
\end{tabular}

\section{From Sperry north to northwest corner of quadrangle.}

\begin{tabular}{|c|c|c|c|}
\hline 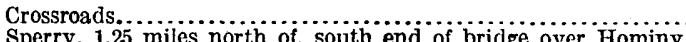 & 361826.5 & 955929.4 & 3,950 \\
\hline Creek; top of nut on east cylinder pier..................... & 361855.5 & $95 \quad 5929.4$ & \\
\hline 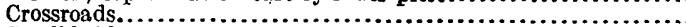 & 361950.9 & 955928.6 & \\
\hline Small bridge........... & 362026.5 & 955928.5 & \\
\hline Crossroads..... & 362103.7 & 955938.6 & \\
\hline T road south ........ & 362155.9 & 955928.5 & \\
\hline Bird Creek, center of br & $\begin{array}{lllll}36 & 23 & \mathbf{4 3} .1\end{array}$ & 955928.4 & 10,8 \\
\hline 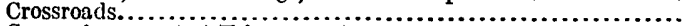 & 362432.6 & $95 \quad 5928.8$ & 5,0 \\
\hline Corner fence post at $T$ fence west. & $\begin{array}{llll}36 & 25 & 42.7\end{array}$ & 955905.0 & \\
\hline Road corner, roads south and east, & 362616.8 & 955904.7 & \\
\hline 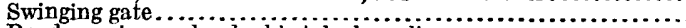 & 362636.3 & 955856.6 & 2,0 \\
\hline 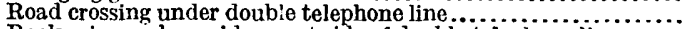 & 362735.0 & 955928.6 & \\
\hline Rock cairn on bare ridge, east side of double telephone line.. & 362809.0 & 955928.6 & \\
\hline Road crossing at double telephone line .... & 362844.5 & 955928.8 & \\
\hline
\end{tabular}

Magnetic declination for northwest quarter of quadrangle, $9^{\circ} 15^{\prime}$ E.; for southwest quarter of quadrangle, $9^{\circ} \mathrm{E}$. 
FORAKER QUADRANGLE.

West along highways in northwest corner of quadrangle. (By B. H. Yoakum In 1916.)

\begin{tabular}{|c|c|c|c|}
\hline Station. & Latitude. & Longitude. & $\begin{array}{l}\text { Distance } \\
\text { between } \\
\text { stations. }\end{array}$ \\
\hline 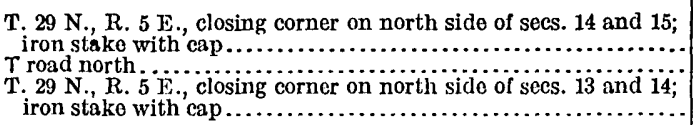 & $\begin{array}{ccc}\bullet & \prime & \prime \prime \\
36 & 59 & 56.2 \\
36 & 59 & 56.2 \\
36 & 59 & 56.3\end{array}$ & $\begin{array}{lcc}\circ & \prime & \prime \prime \\
96 & 44 & 28.3 \\
96 & 44 & 11.6 \\
96 & 43 & 22.6\end{array}$ & $\begin{array}{r}\text { Feet. } \\
2,605 \\
1,355 \\
3,973\end{array}$ \\
\hline
\end{tabular}

West along highways near west border of quadrangle.

\begin{tabular}{|c|c|c|c|}
\hline $\begin{array}{l}\text { T. } 28 \text { N., R. } 5 \text { E., corner of secs. } 25,26,35 \text {, and } 36 \ldots \ldots \ldots \\
\text { T. } 28 \text { N., R. } 5 \text { E., quarter corner between secs. } 26 \text { and } 35 \text {; iron st }\end{array}$ & 365207.9 & $9643 \quad 30.2$ & \\
\hline 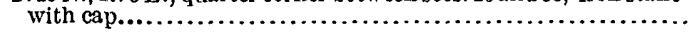 & 365208.0 & 964402.8 & 2,640 \\
\hline
\end{tabular}

Magnetic declination along north border in northwest corner of quadrangle, $10^{\circ}$ $10^{\prime} \mathrm{E}$.

West along highways near south bordex of quadrangle.

Tps. 26 and 27 N., Rs. 5 and 6 E.; General Land Office iron post. . T.ps. 26 and 27 N., R. 5 F., corner of secs. 1, 2, 35, and 36 , fence line

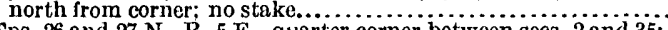
Tps. 26 and 27 N., R. 5 E., quarter corner between secs. 2 and 35 ;

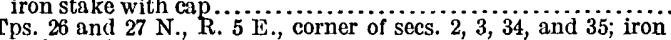

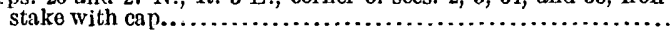

\section{GRAY HORSE QUADRANGLE.}

North along highways and Atchison, Topeka \& Santa Fe Ry. near west border of quadrangle. (By B. H. Yoakum in 1916.)

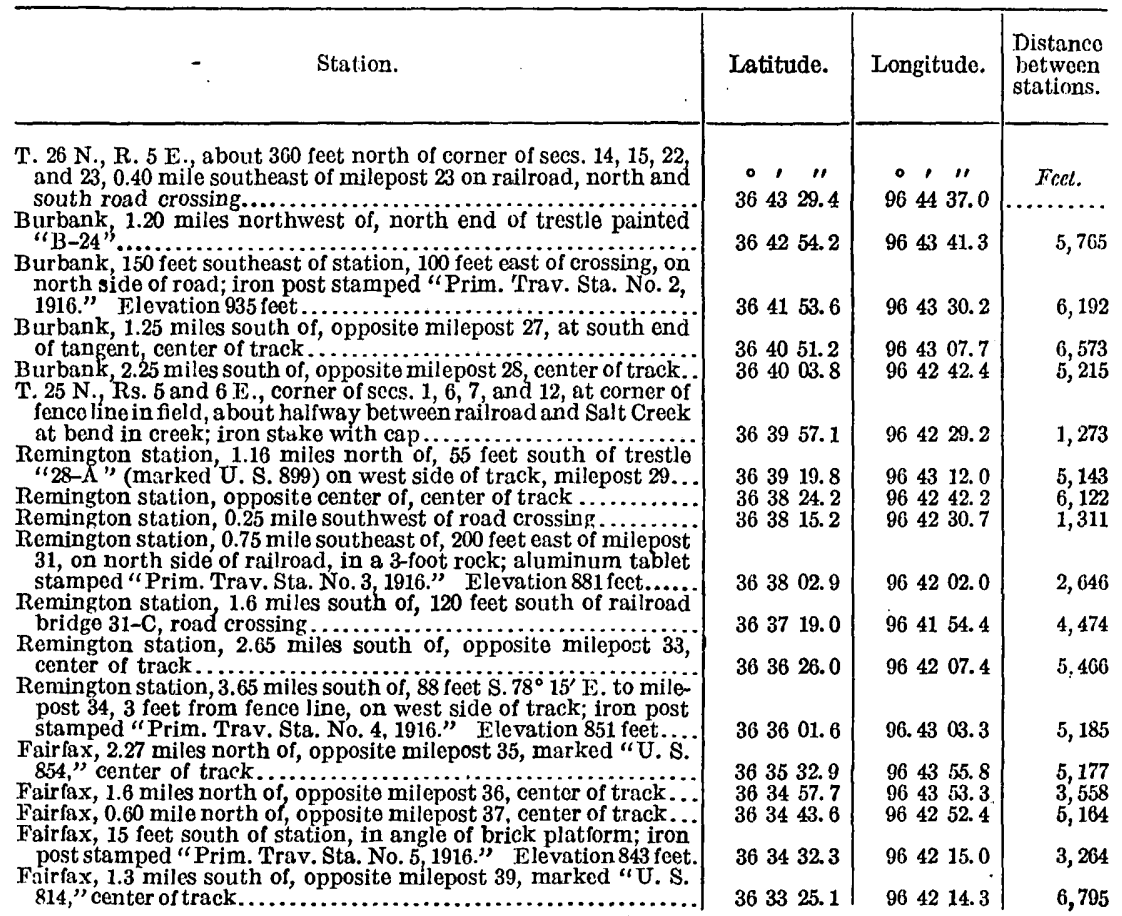


GRAY HORSE QUADRANGLE-Continued.

North along highways und Atchison, Topeka \& Santa Fo Ry. near west border of quadrangle-Continued.

\begin{tabular}{|c|c|c|c|}
\hline Station. & Latitude. & Longitude. & $\begin{array}{l}\text { Distance } \\
\text { between } \\
\text { stations. }\end{array}$ \\
\hline 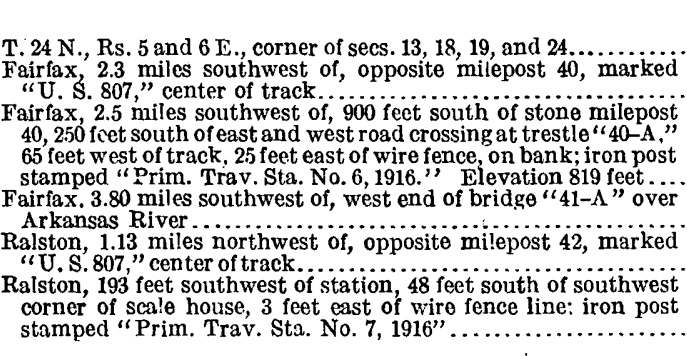 & $\begin{array}{llc}\circ & \prime & \prime \prime \\
36 & 33 & 01.2 \\
36 & 32 & 34.6 \\
36 & 32 & 27.4 \\
36 & 31 & 51.0 \\
36 & 31 & 27.2\end{array}$ & $\begin{array}{ccc}\circ & \prime & \prime \prime \\
96 & 42 & 36.3 \\
96 & 42 & 31.0 \\
96 & 42 & 37.6 \\
96 & 43 & 49.6 \\
96 & 43 & 58.5\end{array}$ & $\begin{array}{l}\text { Feet. } \\
\quad 3,0 \div 5 \\
2,713\end{array}$ \\
\hline
\end{tabular}

East along highways near south border of quadrangle.

Ralston, 0.62 mile east of, south end of wagon brilge over Arkansas River.

Tps. 23 and 24 N., Rs. 5 and 6 F., corner of secs. $1,6,31$, and 36, in center of east and west road: General Land Office iron post..

Tps. 23 and 24 N., R. 6 E., corner of secs. $5,6,31$, and 32; crossroads. Tps. 23 and 24 N., R. 6 E., corner of secs. $4,5,32$, and 33 ; iron post with cap

Tps. 23 and 24 N., R. 6 E., quarter corner between secs. 4 and 33 ;

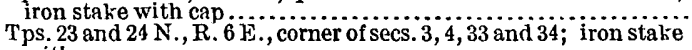
with cap.

Tps. 23 and 24 N., R. 6 F., quarter corner between secs. 3 and 34 ;

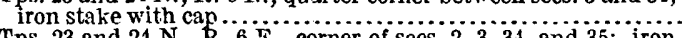

Tps. 23 and 24 N., R. 6 . ., corner of secs. $2,3,34$, and 35 ; iron

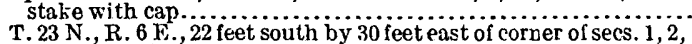
T. 23 N., R. 6 F., 22 feet south by 30 feet east of corner of secs. 1,2 ,
35 , and 36 ; iron post stamped "Trim. Trav. Sta. No. 8,1916 " .. Tps. 23 and 24 N., R. 6 F., quarter corner between secs. 1 and 36 ; iron stake with cap

Tps. 23 and 24 N., Rs. 6 and 7 F., corner of secs. $1,6,31$, and 36 ;

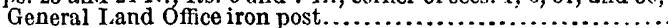

Tps. 23 and $24 \mathrm{~N}$., R. $7 \mathrm{E}$, corner of secs. $5,6,31$, and 32 ; iron stake with cap..................................................

Tps. 23 and 24 N.,, 7 . 7 F, quarter comer between secs. 3 and 34 ;

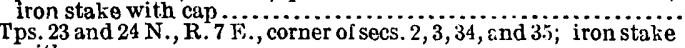

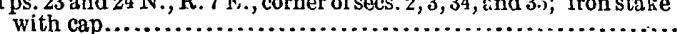

Tps. 23 and $24 \mathrm{~N} ., \mathrm{R} .7 \mathrm{E}$. , quarter corner between secs. 2 and $35 ;$

Tps. 23 and $24 \mathrm{~N}$., $\mathrm{R}$. $7 \mathrm{E}$. corner of secs. $1,2,35$, and 36 ; iron stake with cap.

Tps. 23 and $24 \mathrm{~N}, \mathrm{R} .7 \mathrm{E} .1,110$ feet east of quarter corner between secs. 1 , and 36 on west side of road, 15 feet west of "hlack-jack" tree, on township line; iron post stamped "Prim. Trav. Sta. No. $9,1916^{\prime \prime}$

\section{5}

363025.0

363025.4

$\begin{array}{lll}36 & 30 & 25.7\end{array}$

$\begin{array}{ll}36 & 3025.8\end{array}$

363025.9

$\begin{array}{lll}36 & 30 & 25.7\end{array}$

$\begin{array}{ll}36 & 3025.3\end{array}$

363026.0

363026.5

363026.9

363026.3

363025.7

363025.6

363025.9

363025.1

363024.8
$96 \quad 4328.9$

3,268

964236.6 964133.9

$96 \quad 4028.2$

963955.8

963923.2

963350.8

963818.5

963713.6

963641.6

963609.4

963509.9

063228.3

363156.0

963123.6

963051.2

963005.4

3,745
4,622 5,119 5,361 2,648 $2,6 \subset 0$ 2,636 2,644 5, 205 2,606 $2,6: 5$ 4,852 13,190 2,642 2,642 2,638

\section{North along highways near east border of quardangle.}

T. 24 N., R. 7 T., west side of sec. 24 , on west bank of Hominy Creek, 100 feet north of sharp bend in creek to west, 10 feet north of blazed white-oak tree marked "U.S. G.S. Prim. Trav. Line"

T. 24 N., R. $7 \mathrm{E}$., quarter corner between secs. 23 and 24 at fence

T.24 N., R. $7 \mathrm{~F}$. , quarter corner between secs. 13 and 14 ; iron stake

T. 24 N., R. 7 E., corner of secs. 11, 12, 13, and 14; iron stake with T. 24 N. R. 7 E. corner of secs. $1,2,11$ and 12 ; iron stake with cap T. 24 N., R. 7 E., quarter corner between secs. 1 and 2 ; iron stake w. with cap., $\mathrm{R} .7 \mathrm{E}$. , north side of secs. 1 and 2 , correction corner; iron T. 25 N., R. 7 E., corner of secs. $25,26,35$, and 36 ; iron sta 3 with T. 25 N., $\mathrm{R} .7$ E. , quarter corner between sees. 25 and 26 ; iron stake with cap.
363226.1

$\begin{array}{lll}36 & 32 & 34.7\end{array}$ 363326.2

363352.1 $3634 \quad 44.1$ 363510.2 363536.1 363628.9 363655.3
$96 \quad 30 \quad 47.1$

12,735

$96 \quad 3052.1$ 963052.5

956

963052.7 963052.9 963053.0 963053.0 963040.1 963039.4 
GRAY HORSE QUADRANGLE-Continued.

North along highways near east border of quadrangle-Continued.

\begin{tabular}{|c|c|c|c|}
\hline Station. & Jatitude. & Longitude. & $\begin{array}{l}\text { Distance } \\
\text { between } \\
\text { stations. }\end{array}$ \\
\hline 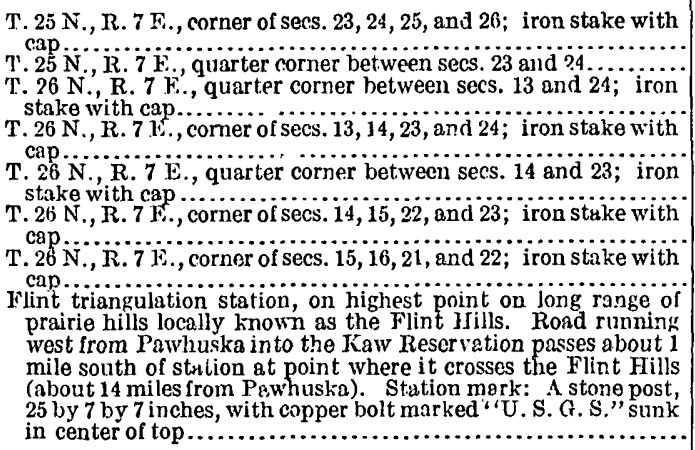 & $\begin{array}{ccc}\circ & \prime & \prime \prime \\
36 & 37 & 21.5 \\
36 & 37 & 45.8 \\
& & \\
36 & 43 & 26.9 \\
36 & 43 & 26.5 \\
36 & 43 & 26.8 \\
36 & 43 & 27.0 \\
36 & 43 & 27.8\end{array}$ & $\begin{array}{cccc}0 & \prime & \prime \prime \\
96 & 30 & 39.1 \\
96 & 30 & 36.5 \\
96 & 30 & 04.0 \\
96 & 30 & 36.3 \\
96 & 31 & 09.1 \\
96 & 31 & 41.1 \\
96 & 32 & 46.4\end{array}$ & $\begin{array}{r}\text { Fect. } \\
2,645 \\
2,472 \\
\\
\\
2,631 \\
2,670 \\
2,629\end{array}$ \\
\hline
\end{tabular}

West along highways through center of quadrangle.

T. 25 N., R. 7 E., corner of secs. $15,16,21$ and 22 ; iron stake with T. 25 N., R. 7 E., quarter corner between secs. 16 and 21 ; iron stake T. 25 N., R. 7 E., corner of secs. $16,17,20$, and $21 ;$ iron stake with T. 25 N., R. 7 E., corner of secs. $17,18,19$, and $20 ;$ iron stake with T. 25 N., R. 7 i., quarter corner between secs. is and 19 ; iron

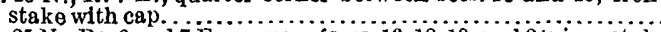
T. 25 N., Rs. 6 and $7 \mathrm{E}$. , corner of secs. $13,18,19, \ldots$ and $24 ;$ iron stake with cap.

T. 25 N., R. 6 E., 1 foot north of corner of secs. $13,14,23$, and 24 ; iron post stamped "Prim. Trav. Sta., No. 12, 1916"........... T. 25 N., R. 6 E., corner of secs. $17,18,19$, and 20 , at T road south

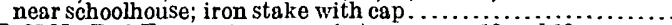
T. 25 N., R. 6 E., quarter corner between secs. 18 and $10 . \ldots \ldots \ldots . . . . .$.

\begin{tabular}{|c|c|c|}
\hline $\begin{array}{lll}36 & 38 & 12.0\end{array}$ & $96 \quad 32 \quad 48.4$ & \\
\hline $\begin{array}{llll}36 & 38 & 12.4\end{array}$ & 963320.6 & 2,625 \\
\hline $\begin{array}{lll}36 & 38 & 12.9\end{array}$ & $96 \quad 3352.9$ & 2,628 \\
\hline $\begin{array}{llll}36 & 38 & 13.4\end{array}$ & $\begin{array}{lll}96 & 34 & 58.0\end{array}$ & 5,308 \\
\hline $\begin{array}{lll}36 & 38 & 12.7\end{array}$ & 963530.2 & 2,618 \\
\hline $\begin{array}{lll}36 & 38 & 12.5\end{array}$ & 963602.0 & 2,592 \\
\hline $\begin{array}{lll}36 & 38 & 12.8\end{array}$ & 963706.5 & 5,259 \\
\hline $\begin{array}{lll}36 & 38 & 13.3 \\
36 & 38 & 13.2\end{array}$ & $\begin{array}{lll}96 & 41 & 25.1 \\
96 & 41 & 57.6\end{array}$ & $\begin{array}{r}21,082 \\
2,646\end{array}$ \\
\hline
\end{tabular}

Magnetic declination for north border of quadrangle, $9^{\circ} 42^{\prime} \mathrm{E}$.; for south border of quadrangle, $9^{\circ} 48^{\prime} \mathrm{E}$; ; for east border of quadrangle, $9^{\circ} 46^{\prime} \mathrm{E}$; ; for west border of quadrangle, $9^{\circ} 55^{\prime} \mathrm{E}$.; for center of quadrangle, $9^{\circ} 49^{\prime} \mathrm{E}$.

\section{Along highways in the extreme southwest corner of quadrangle.}

Tps. 23 and 24 N., R. 5 E., corner ofsecs. 2, 3, 34, and 35, crossroads; Tps. 23 and 24 N., R. 5 E., quarter corner between sees. 2 and 35 , at Tps. 23 and 24 N., R. 5 E., quarter corner between secs. 2 and 35 , at

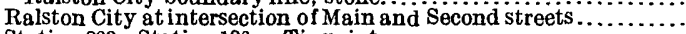
Station $833=$ Station 126 . Tie point.

\begin{tabular}{rrr|rrr|r}
36 & 30 & 25.2 & 96 & 44 & 45.1 & $\ldots \ldots \ldots$ \\
& & & & & \\
36 & 30 & 25.0 & 96 & 44 & 12.9 & \\
36 & 30 & 25.1 & 96 & 44 & 08.2 & \\
36 & 30 & 13.9 & 96 & 43 & 57.2 & 631 \\
& & & & 384 \\
& & & & \\
\hline
\end{tabular}

Magnetic declination for southwest corner of quadrangle, $10^{\circ} 01^{\prime} \mathrm{E}$.

Along highways near west center of quadrangle.

\begin{tabular}{|c|c|c|c|}
\hline 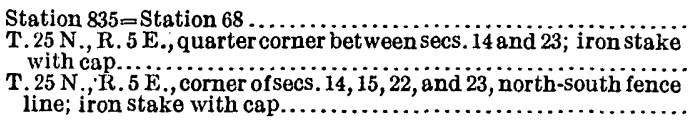 & $\begin{array}{lll}36 & 38 & 15.48 \\
36 & 38 & 13.2 \\
36 & 38 & 13.1\end{array}$ & $\begin{array}{l}964231.09 \\
964406.8 \\
964439.1\end{array}$ & $\begin{array}{l}7,803 \\
2,635\end{array}$ \\
\hline
\end{tabular}


HOMINY QUADRANGLE.

North along highways near west border of quadrangle, north half. (By W. F. Hicks in 1916.)

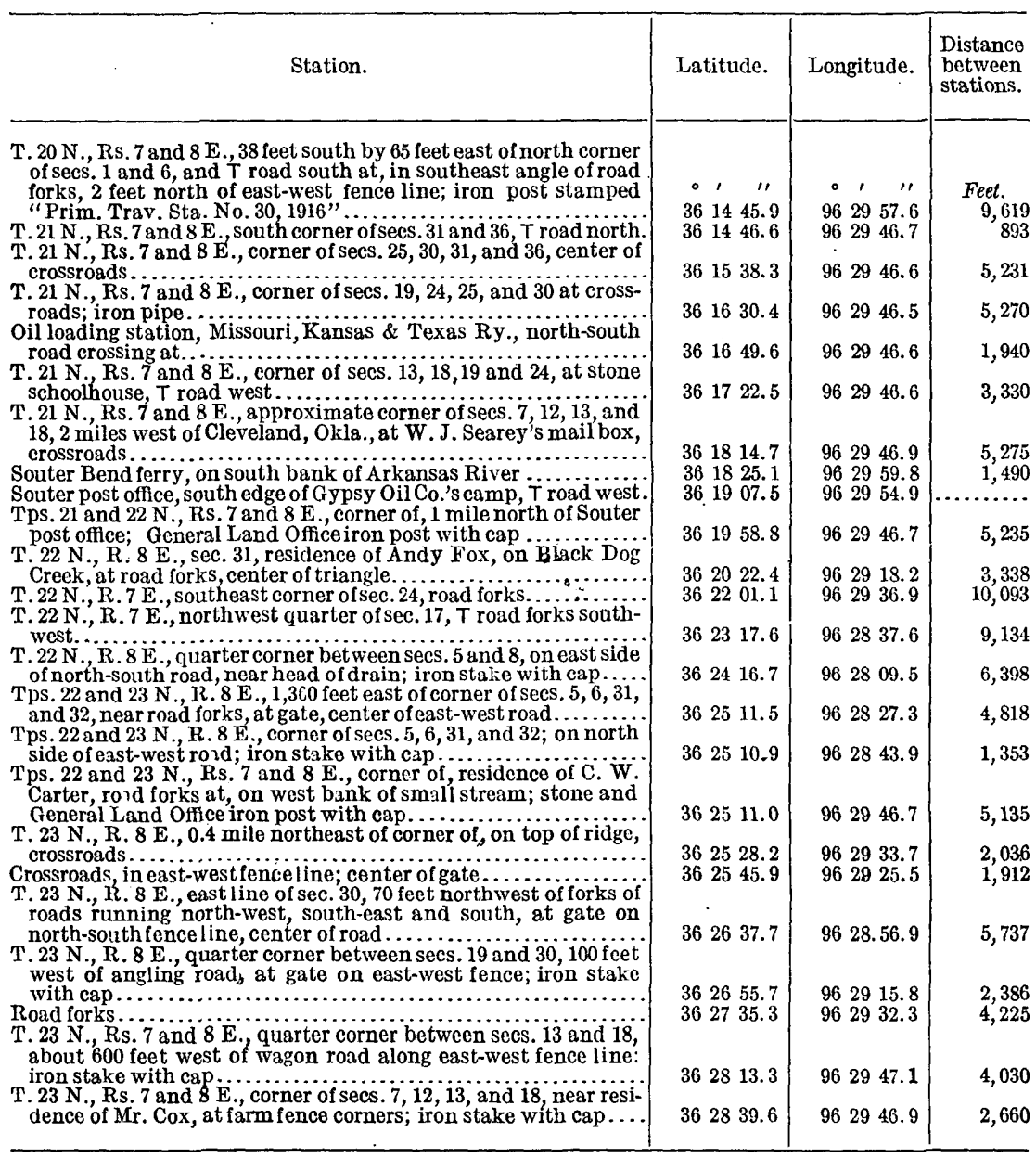

Magnetic declination on west border of quadrangle, $9^{\circ} 36^{\prime} \mathrm{E}$.

Near west border of quadrangle.

\begin{tabular}{|c|c|c|c|}
\hline 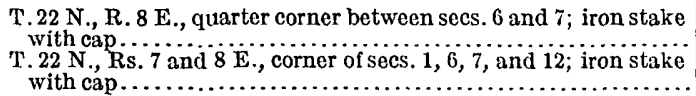 & 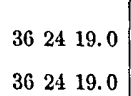 & $\begin{array}{llll}96 & 29 & 14.3 \\
96 & 29 & 46.6\end{array}$ & 2,639 \\
\hline
\end{tabular}

Along highways near south border of quadrangle (southwest corner).

\begin{tabular}{|c|c|c|c|}
\hline 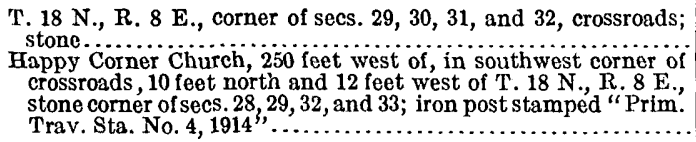 & $\begin{array}{lll}36 & 00 & 10.9\end{array}$ & $\begin{array}{c}962939.4 \\
.\end{array}$ & 5,350 \\
\hline
\end{tabular}


HOMINY QUADRANGLE-Continued.

North along highways near west border of quadrangle.

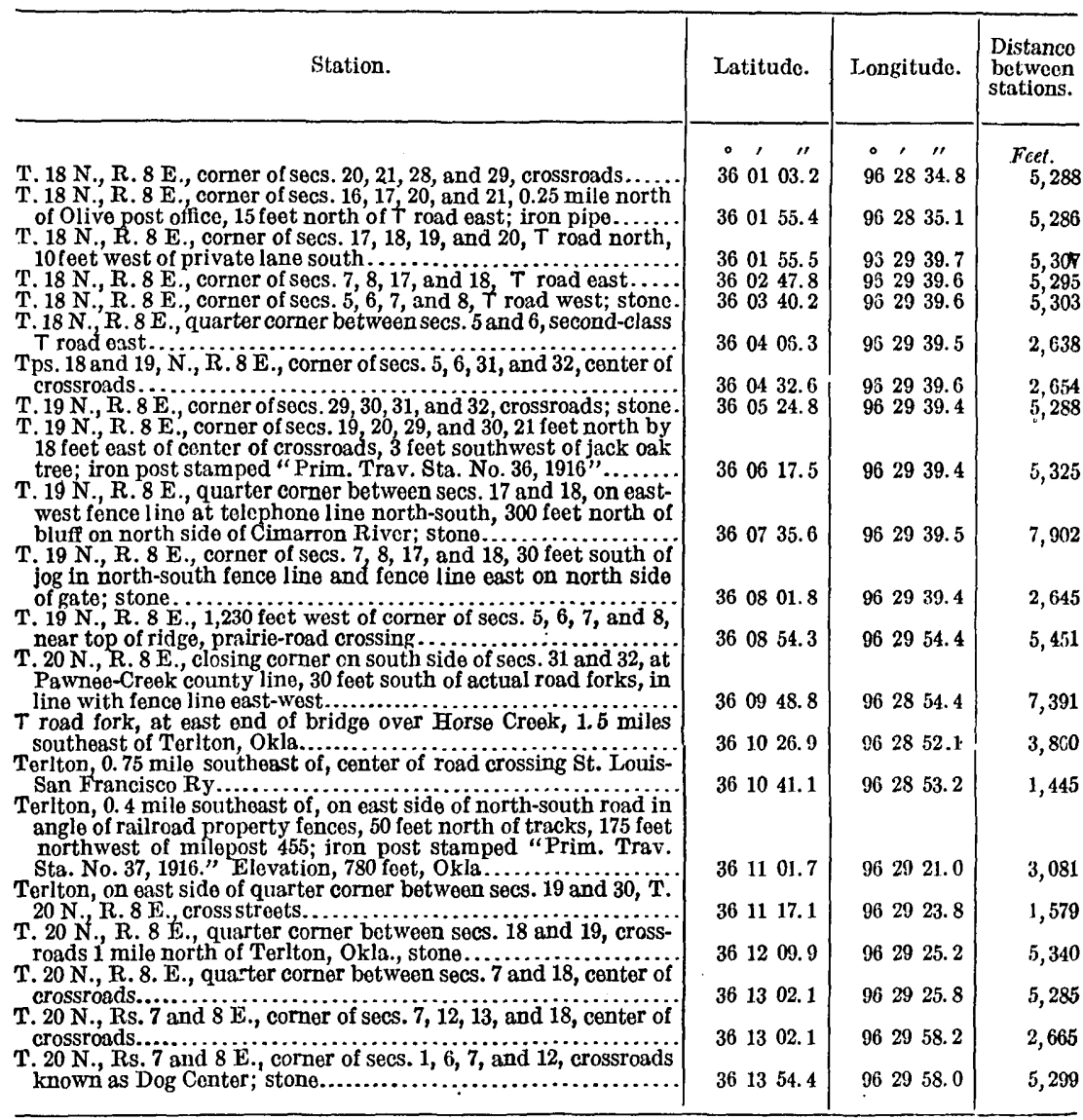

Magnetic declination along west border of quadrangle, south half, $9^{\circ} \cdot 36^{\prime} \mathrm{E}$.

North along public roads from southeast corner of quadrangle to West Tulsa. (By B. H. Yoakum In 1916.)

T. 18 N., R. $12 \mathrm{~J}$, corner of secs. $27,28,33$, and 34 , Tulsa-Creek

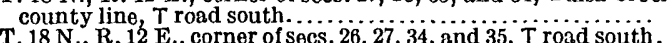

T. 18 N., R. 12 E., corner of secs. $2,3,10$, and 11 ; iron post at center

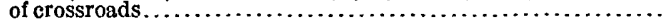

Tps. 18 and 19 N.,,$\ldots 12$ E., corner of secs. $2,3,34$, and $35 ;$;ron post T. 19 N., $R .12$ E., corner of secs. $26,27,34$, and 35 ; stone at crossT. 19 N.,,$\ddot{R} .12$ E., quarter corner botween secs. 26 and $27, \mathrm{~T}$ road

T. 19 N., R. 12 E., corner of secs. $22,23,26$, and 27 ; stone at $\mathrm{T}$ road

T. 19 N., R. 12 E., 30 foet north by 30 feet west of crossroads, at corner of secs. 10, 11, 14, and $15,0.5$ mile southwest of West Tulsa, 245 feet south of St. Louis-San Francisco Ry. crossing; iron post stamped "Prim. Trav. Sta. No. 61, 1916"

West Tulsa station, 120 feet south of, Mitchell Street crossing of St. Louis-San Francisco Ry., center of middle track
$3600 \quad 10.7$

$\begin{array}{lll}36 & 00 & 10.8\end{array}$

$\begin{array}{llll}36 & 03 & 40.0\end{array}$

360431.3

360523.6

$\begin{array}{lll}36 & 05 & 49.8\end{array}$

360616.0

360801.0

360815.6 $\begin{array}{lll}96 & 01 & 45.5\end{array}$

$96 \quad 0041.2$

$\ddot{5}, \ddot{290}$

960041.2

$9600 \quad 41.2$

$\begin{array}{llll}96 & 00 & 41.1\end{array}$

$9600 \quad 41.1$

$9600 \quad 40.8$

$\begin{array}{lll}96 & 00 & 40.8\end{array}$

10,614

960030.2
5,179

5,290

2,648

2,651

1,713 
HOMINY QUADRANGLE-Continued.

Along St. Louis-San Francisco Ry. from West Tulsa to Mannford.

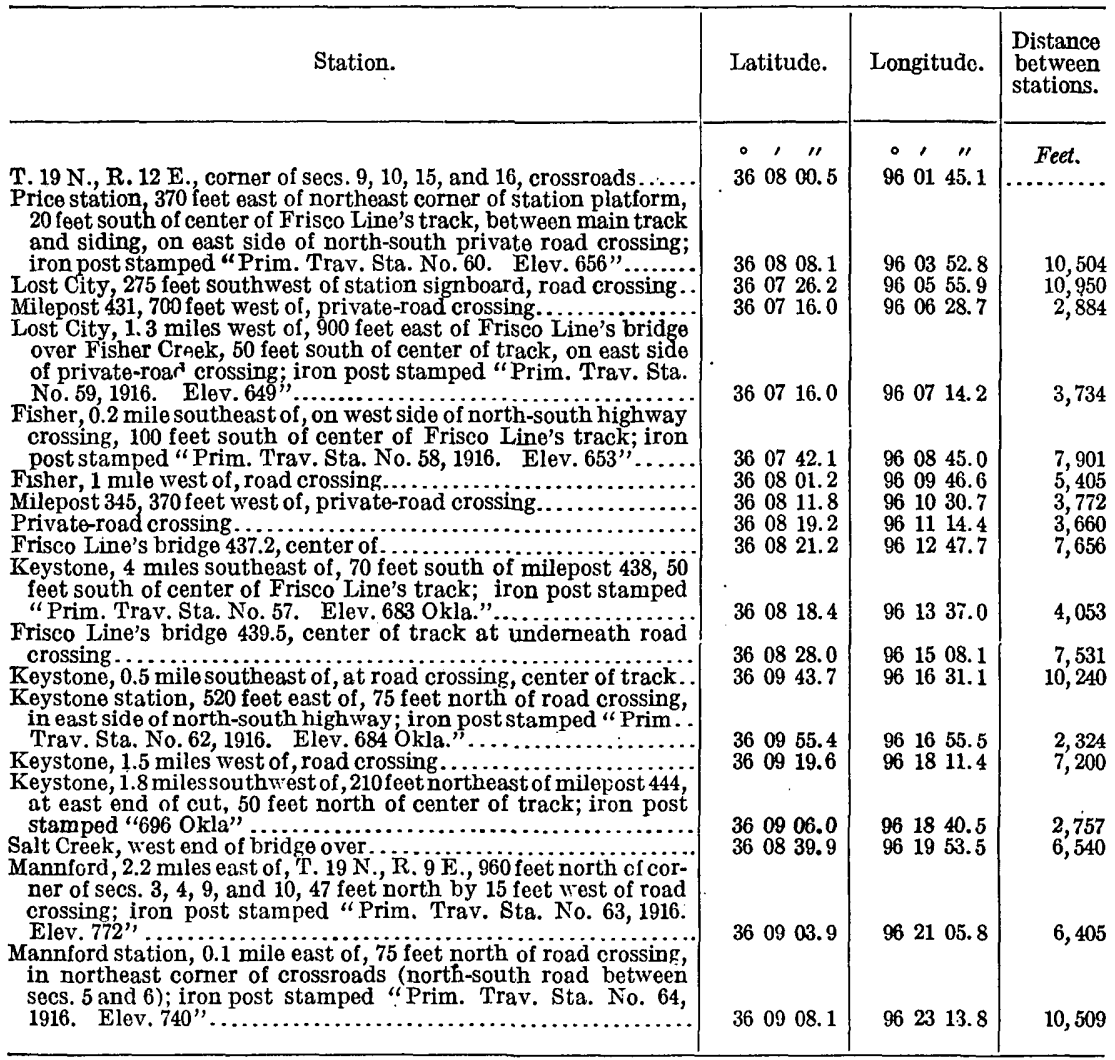

From Mannford south and west along public highway to west border of quadrangle.

Mannford, T road west at brick schoolhouse, corner of secs. 5,6 ,

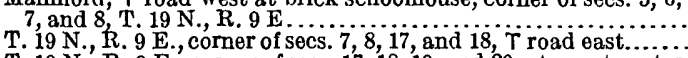
T. 19 N., R. 9 E., corner of secs. $17,18,19$, and 20 ; stone at center

T. 19 N., Rs. 8 and 9 E., corner of secs. $13,18,19$, and 24 , center of east-west road at $T$ road south.

T. 19 N., R. $8 \mathrm{E}$, comer of secs. $13,14,23$, and 24,7 road south. T. 19 N., R. 8 E., quarter corner between secs. 14 and 23 , in center T. $19 \mathrm{~N}$,, $\mathrm{R} .8 \mathrm{E}$., corner of secs. $14,15,22$, and 23 , near vacant house; 30 feet northeast of fence corner.

T. 19 N., R. 8 E., near center of sec. 22 , road forks.

T. 19 N., R. 8 E., near quarter corner between secs, 21 and 28 , center of east-west road at gate in north-south fence line. .

T. 19 N., R. 8 E., approximate corner of secs. $20,21,28$, and 29 , midway between private roads north and south, center of eastwestroad.

T. 19 N., R. 8 E., corner of secs. $19,20,29$, and 30,21 feet north by 18 feet east of center of crossroads; iron post stamped "Prim. Trav. Sta. No. 36, 1916"
$3608 \quad 54.4$ 360802.1

$\begin{array}{lll}36 & 07 & 03.7\end{array}$

$\begin{array}{lll}36 & 07 & 09.8\end{array}$

$\begin{array}{lll}36 & 07 & 09.8\end{array}$

$\begin{array}{lll}36 & 07 & 09.7\end{array}$

$\begin{array}{lll}36 & 07 & 09.7\end{array}$

$\begin{array}{lll}36 & 06 & 45.2\end{array}$

$3606 \quad 17.4$

$\begin{array}{lll}36 & 06 & 17.2\end{array}$

$3606 \quad 17.5$

\begin{tabular}{lll|l}
96 & 23 & 14.3 & 1,390 \\
96 & 23 & 14.1 & 5,284 \\
96 & 23 & 14.0 & 5,301 \\
96 & 24 & 17.3 & 5,190 \\
96 & 25 & 21.7 & 5,283 \\
96 & 25 & 54.0 & 2,647 \\
96 & 26 & 26.3 & 2,648 \\
96 & 26 & 56.6 & 3,513 \\
96 & 28 & 02.8 & 6,114 \\
96 & 28 & 35.3 & 2,659 \\
96 & 29 & 39.4 & 5,262 \\
\hline
\end{tabular}


HOMINY QUADRANGLE-Continued.

Along public road from Keystone south to Phantom triangulation station.

\begin{tabular}{|c|c|c|c|}
\hline Station. & Latitude. & Longitude. & $\begin{array}{l}\text { Distance } \\
\text { between } \\
\text { stations. }\end{array}$ \\
\hline 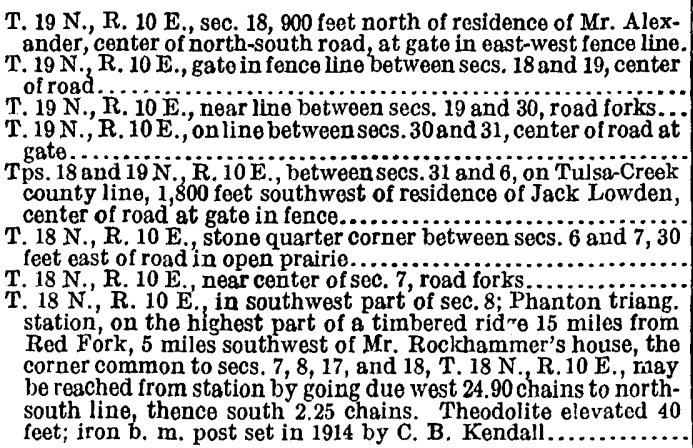 & $\begin{array}{ccc}\circ & \prime & \prime \prime \\
36 & 07 & 50.4 \\
36 & 07 & 10.0 \\
36 & 06 & 19.8 \\
36 & 05 & 25.4 \\
36 & 04 & 32.9 \\
36 & 03 & 40.7 \\
36 & 03 & 08.6\end{array}$ & \begin{tabular}{cccc}
0 & $\prime$ & \multicolumn{1}{c}{} \\
96 & 17 & 18.6 \\
96 & 17 & 16.4 \\
96 & 17 & 24.7 \\
96 & 17 & 10.8 \\
96 & 17 & 01.0 \\
96 & 17 & 18.0 \\
96 & 17 & 19.9
\end{tabular} & $\begin{array}{r}\text { Feet. } \\
\ldots \ldots \ldots \\
4,090 \\
5,121 \\
5,613\end{array}$ \\
\hline
\end{tabular}

East along public hlghways from west border of trlangle to Prue.

T. 20 N., Rs. 7 and 8 E., 38 ft. south by 65 feet east of north corner of secs. 1 and 6 , at $T$ road south, in southeast angle of road forks, 2 feet north of east-west fenceline; iron post stamped "Prim. Trav. Sta. No. 30,1916"

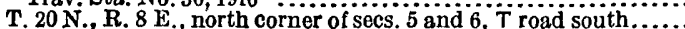

T. 20 N., R. 8 E., stone quarter corner on north side of sec. 5 , fence

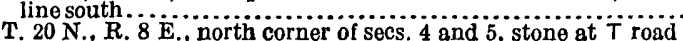
T. 21 N., $\ldots . .1$ E., south corner of secs. 32 and 33 , stone at $T$ road T. $20 \mathrm{~N} ., \mathrm{R} .8 \mathrm{E}$., north corner of secs. 3 and $4, \ldots \ldots \ldots \ldots$ road south........ T. 21 N.,, R. 8 E., north curner of secs. 3 and $4, T$ road south...... Crossroads at Oklahoma Petroleum \& Gasoline Co.'s plant...... T. 20 N., R. 8 E., north corner of secs. 1 and $2, T$ road south....... T. 21 N., R. 8 E., south corner of secs. 35 and 36 , T road north... T. 21 N., R. 8 E., corner of secs. $25,26,35$, and 36 , stone at crossT. 21 N. R. 8 E... quarter corner between secs. 25 and 36 , in center of road at fence line north and south; stone.................. T. 21 N., Rs. 8 and $9 \mathrm{E}$., corner of secs. $25,30,31$, and 36,90 feet

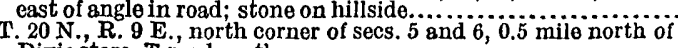
Dixiestore, T road south.

T. 20 N. R. 9 E., quarter corner on north side of sec. 5,450 feet west of frame house, on east bank of creek; stone..............
361445.9 361446.1

$36 \quad 14 \quad 45.9$

$36 \quad 1445.8$

$36 \quad 14 \quad 45.7$

$3614 \quad 45.4$

$\begin{array}{lll}36 & 14 & 45.3\end{array}$

$36 \quad 1445.3$

361445.1

361445.3

36 is 37.2

361536.4

361536.9

$36 \quad 14 \quad 45.1$

$36 \quad 14 \quad 45.1$
962957.6 962355.1

$5,1.19$

$\begin{array}{lll}96 & 28 & 23.0\end{array}$

2,628

962751.0

2,624

962738.0

96 $26 \quad 46.0$

962633.9

962513.7

$\begin{array}{llll}96 & 24 & 37.3\end{array}$

962425.0

1003

1,063
4,257

995

6,568

2,980

1,015

962425.6

5,245

$96 \quad 2353.4$

2, 640

962320.8

2,673

962232.4

6, 565

$96 \quad 2200.2$

2,640

Southeast along Missouri, Kansas \& Texas Ry. from polut about 2.5 miles west of Prue to OsageTulsa county-line post near Appalachia. (By B. H. Yoakum in 1916.)

Prue, 1.6 miles northwest of, 400 feet east of milepost 251 , on north side of railroad, near right of way fence line at former north and south road crossing, in southeast angle of fence corner; iron post stamped "Prim. Trav. Sta. No.65,1916. Elev. 702 Okla."......

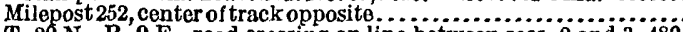

T. 20 N., R. 9 E., road crossing on line between secs. 2 and 3,480

feet west of Pruestation..................................... 0.9 mile east oi on quarter corner between secs. 1 and $2, T .20 \mathrm{~N}$., R. $9 \mathrm{E}$., on west side of private wagon road; iron post stamped "Prim. Trav. Sta. No 66, 1916. Elev, 692 Okla," post stamped "Prim. Trav. (This post was reset and new elevation established.)

Milepost 255, 380 feet east of, 70 feet west of trestle 255.1 over Rock Creek, private-road crossing

T. 20 N, R. 10 E., corner of secs. $5,6,7$, and 8,180 feet south of,

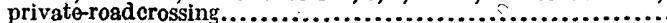

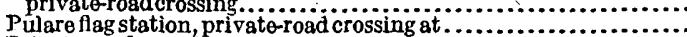

Privateroad crossing............

A ppalachia, 1.5 miles northwest of, in SE. \& sec. 29, T. 20 N. north of gate in property-line fence; iron post stam ped "Prim. Trav. Sta.' No. 67, 1916 . Elev. 678 Okla."

Appalachia, 0.25 milo northwest of, in southwest angle of road crossing Missouri, Kansas \& Texas Ry.; Tulsa-Osage countyline post.

361418.9 361400.9

361402.6

361406.0

$\begin{array}{lll}36 & 13 & 53.7\end{array}$

$\begin{array}{lll}36 & 13 & 50.6\end{array}$

361301.8

$\begin{array}{lll}36 & 12 & 07.1\end{array}$

$\begin{array}{lll}36 & 10 & 43.7\end{array}$

$\begin{array}{lll}36 & 09 & 43.1\end{array}$
962050.2 961956.2

6,308 4, 793 961920.0

2, 964

961816.2

5,238

961641.0

7,899

$96 \quad 1609.3$

$96 \quad 1429.7$

$2,61 y$

9,538

961419.4

5,508 .

961529.1

10,182

961534.0 
HOMINY QUADRANGLE-Continued.

Tulsa to Sperry north along public roads.

An error of about 2.4" in latitude and 1.6" in longitude exists between Tulsa and Sperry.

\begin{tabular}{|c|c|c|c|}
\hline Station. & Latitude. & Longitude. & $\begin{array}{l}\text { Distance } \\
\text { between } \\
\text { stations. }\end{array}$ \\
\hline 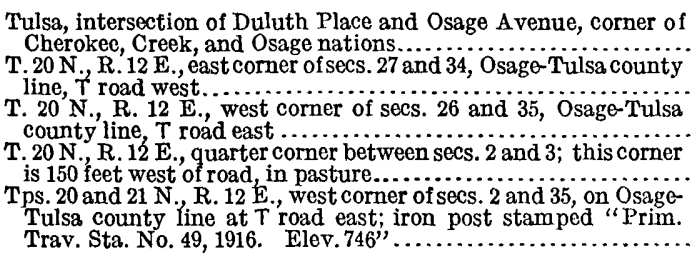 & $\begin{array}{ccc}\circ & \prime & \prime \prime \\
36 & 09 & 40.6 \\
36 & 10 & 21.6 \\
36 & 10 & 37.0 \\
36 & 14 & 14.1\end{array}$ & $\begin{array}{ccc}\circ & \prime & \prime \prime \\
96 & 00 & 03.6 \\
96 & 00 & 03.8 \\
96 & 00 & 03.9 \\
96 & 00 & 04.6\end{array}$ & $\begin{array}{l}\text { Feet. } \\
4,140 \\
1,560\end{array}$ \\
\hline
\end{tabular}

From point 1.25 miles south of Sperry, southwest along highways to Missouri, Kansas \& Texas Ry., about 1 mile west of Wekiwa.

Osage-Tulsa county line, center of east and west road.............

T. 21 N., R. 12 E., quarter corner between secs. 21 and 22 , crossroads..

T. 21 N., R. 12 E., center of sec. 21, crossroads at Delaware school-

T. 21 N., R. 12 E., quarter corner between secs. 20 and 21 , private T. 21 N., R. 12 E., quarter corner between secs. 19 and $20, T$ road

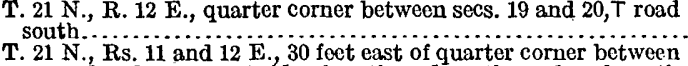
secs, 19 and 24 , on east side of north and south road and south side of T road east; iron post stamped "Prim. Trav. Sta. No. 52,

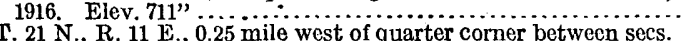
T. 21 N., R. 11 E., 0.25 mile west of quarter corner between secs. public road, in root of 30-inch post-oak tree; two 20-penny wire nails bent across, painted "U.S. 680 B. M."

T. 21 N., R. 11 E., quarter corner between secs. 26 and 27 .

T. 21 N., R. 11 E., 0.5 mile east of quarter corner between sess. 27 and 28,10 feet north of gate in public road, 8 feet southwest of three-way fence corner; iron post stamped "Prim. Trav. Sta. No. 53, 1916. Elev. 732".............................

T. 21 N., R. 11 E., near quarter corner between secs. 33 and 34 , on north bank of Delaware Creek, east side of public road, in top of hickory stump; two 20-penny nails bent across...

T. 21 N., R. 11 E., near center of sec. 33 , at east and west fence and telephone line, center of gate in public road.

Tps. 20 and 21 N., R. 11 E., 500 feet east of quarter corner on south side of sec. 33, 300 feet north of W.C. Heaton's residence, on south side of road at gate; chiseled square in top of stone marked " $U$. S. 772.8 B. M

T. 20 N., R. 11 E., quarter corner between secs. 3 and 4 ............

T. 20 N., R. 11 E., 20 feet south by 35 feet west of corner of secs. $3,4,9$, and 10,20 feet west of gate on public road, on north side of fence; iron post stamped "Prim. Trav. Sta. No. 54, 1916. Elev. 838".

T. 20 N., R. 11 E. quarter corner between secs. 16 and $17 . \ldots .$.

T. 20 N.'R. 11 E., NW. 1 sec. 20,15 feet east of gate on public road, just north of road forks; iron post stamped "Prim. Trav. Sta.

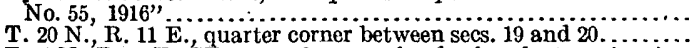

T. 20 N., R. 11 E., SE. $\frac{1}{2}$ sec. 19 , on scuth side of road, at gap in wire fence, in root of 14-inch black-jack oak; railroad tie date nail,

T. 20 N., Rs. 10 and 11 E., 150 feet south of road forks, corner of secs. $19,24,25$, and $30 \ldots \ldots \ldots \ldots \ldots \ldots \ldots \ldots \ldots \ldots \ldots \ldots \ldots \ldots . \ldots \ldots$. east

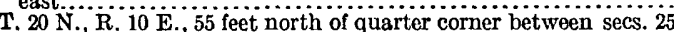
and 36 , at $T$ road east; railroad date nail in root of 20 -inch live-oak tree, marked "U. S. 768 B. M."

Tps. 19 and 20 N., $\dot{R}$. $11 \mathrm{E}$., quarter corner on south side of sec. 36 , at private road west on Osage-Tulsa county line; railroad tio date nail in root of 16 -inch post-oak tree marked "U. S. 777.3 B. M "

Wekiwa, 1 mile west of, 370 feet east of milepost 266,100 feet north of $T$ roadd east, 45 feet north by 25 feet east of rood crossing of Missouri, Kansas \& T exas Ry., 2 feet west of wire fence; iron post stamped "Prim. Trav. Sta. No. 56. Elev. 666"................
365057.0 361653.3 361653.5 $\begin{array}{lll}36 & 16 & 53.6\end{array}$

$\begin{array}{lll}36 & 16 & 53.9\end{array}$

361653.6

3616.01 .4

361601.9

361601.9

$\begin{array}{lll}36 & 15 & 19.8\end{array}$

361509.5

361443.0

$36 \quad 14 \quad 17.4$

361351.1 $\begin{array}{llll}36 & 12 & 33.4\end{array}$

361156.4 $36 \quad 1141.0$

$\begin{array}{lll}36 & 11 & 22.3\end{array}$

361114.9

361115.0

$\begin{array}{lll}36 & 10 & 24.2\end{array}$

$\begin{array}{lll}36 & 40.4\end{array}$

360906.0
3,690

2,634

2,573

5,290

5,280

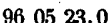

8,459

3,968

960643.9

2,663

960715.2

4,973

960739.6

2,253

$96 \quad 07 \quad 54.3$

960735.9

2,934

2,995

960736.3

$\begin{array}{llll}96 & 08 & 40.2\end{array}$

2,660

9,448

960921.3

$\begin{array}{llll}96 & 09 & 43.1\end{array}$

5,026

2,374

960958.6

2,284

$96 \quad 1046.6$

3,998

961118.5

2,618

961118.6

5,135

961124.4

4, 455

961123.8 
HOMINY QUADRANGLE-Continued.

Along Missourl, Kansas \& Texas Ry. west to Piatt.

\begin{tabular}{|c|c|c|c|}
\hline Station. & Latitude. & Longitude. & $\begin{array}{l}\text { Distance } \\
\text { between } \\
\text { stations. }\end{array}$ \\
\hline 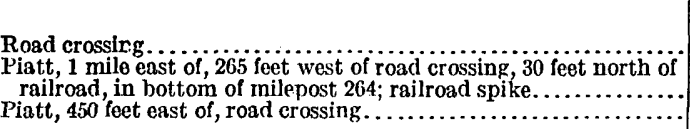 & $\begin{array}{ccc}\circ & \prime & \prime \prime \\
36 & 09 & 00.5 \\
36 & 08 & 55.5 \\
36 & 09 & 09.7\end{array}$ & $\begin{array}{ccc}\bullet & \prime & \prime \prime \\
96 & 12 & 28.6 \\
96 & 13 & 35.9 \\
96 & 14 & 37.2\end{array}$ & $\begin{array}{l}\text { Feet. } \\
5,344 \\
5,547 \\
5,054\end{array}$ \\
\hline
\end{tabular}

From Missourl, Kansas \& Texas Ry. north through center of quadrangle.

T. 20 N., R. 10 E., corner of secs. $5,6,7$, and 8,180 feet north of rail-

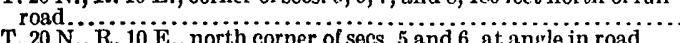
T. 21 N., R. 10 E., quarter corner between secs. 31 and 32 , T road east., R. 10 E., guarter comer between soc. 31 and 32 , T road T. 21 N., R. $10 \mathrm{E}$., corner of secs. $29,30,31$, and 32.

T. 21 N., R. 10 E., quarter corner between secs. 20 and $29 ;$ iron post stamped "Prim. Trav. Sta. No. 68, 1916. Elev. 829"

T. 21 N, R. 10 E., at quarter corner between secs. 8 and 17 southeast corner of schoolhouse yard, district 65 , at three-way fence corner; iron post stamped "Prim. Trav. Sta. No. 69, 1916. Elev.

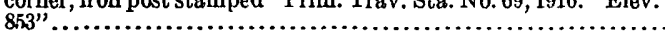

36 1352.4 $3614 \quad 44.6$

$\begin{array}{lll}36 & 15 & 10.7\end{array}$ $\begin{array}{lll}36 & 15 & 36.8\end{array}$

$\begin{array}{ll}36 & 1628.9\end{array}$

$\begin{array}{ll}36 & 18 \\ 12.7\end{array}$
$96 \quad 1609.2$ 961609.0

$96 \quad 15 \quad 51.3$ $96 \quad 1551.3$

961519.0

3,013
2,631

5,896

961518.5

10,493 From pQint 1.25 miles south of Sperry to Missouri, Kansas \& Texas Ry. about 1 mile west of

T. 21 N., R. 10 E., quarter corner between secs. 5 and 8

Tps. 21 and $22 \mathrm{~N}$., R. $10 \mathrm{E}$., quarter corner between secs, 5 and 32 Junction of Buck Creek and Wild HorseCreek, 0.5 mile south of, on

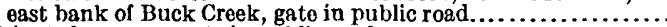
Private lane west, gate in public road at...

T. 22 N., R. 10 E., quarter corner between secs. 20 and 29 , center of east-west road at geto in fenco lime north.

\begin{tabular}{llll|lll|l}
36 & 19 & 04.8 & 96 & 15 & 18.2 & 5,267 \\
36 & 19 & 56.6 & 96 & 15 & 18.1 & 5,238 \\
36 & 21 & 12.8 & 96 & 15 & 21.6 & \\
36 & 21 & 26.2 & 96 & 15 & 16.1 & \\
36 & 21 & 39.6 & 96 & 15 & 19.4 & 1,431 \\
& & & & & 1,378
\end{tabular}

Traverse by W. F. Hicks.

T. 22 N., R. 10 E., in SE. \& sec. 8, road crossing of Hominy Creek. Tps. 22 and 23 N., R. $10 \mathrm{E}$., cormer of secs. $5,6,31$, and 32,170 feet east of ridge road.

$T .23$ N., R. $10 \mathrm{E} .$, in sec. 32 , road fork. .23 N., R. 10 E., on line between sees. 29 and 32,18 -inch oak tree

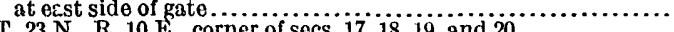

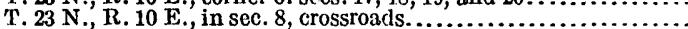

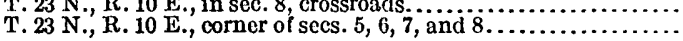

\begin{tabular}{lll|lll|r}
36 & 23 & 29.3 & 96 & 14 & 56.5 & 11,250 \\
36 & 25 & 08.9 & 96 & 15 & 50.8 & 11,005 \\
36 & 25 & 30.2 & 96 & 15 & 32.4 & $2,62$. \\
& & & & & & \\
36 & 26 & 03.8 & 96 & 15 & 32.9 & 3,397 \\
36 & 27 & 45.2 & 96 & 15 & 51.8 & 10,363 \\
36 & 28 & 56.2 & 96 & 15 & 17.9 & 7,691 \\
36 & 29 & 29.7 & 96 & 15 & 50.6 & 4,314
\end{tabular}

Along north border of quadrangle. (By W. F. Hicks.)

T. 23 N., Rs. 7 and $8 \mathrm{E}$., corner of secs. $7,12,13$, and 18 .

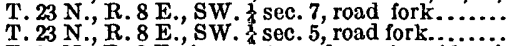

T. 23 N., R. . 23 N., R. 8 E., in sec. 4, road crossing ridge, in saddle..

Gate in north-south fence.

T. 23 N., R. 10 E., SW. s sec. 6,75 feet south of road, in smallopen-

ing; iron post stamped "Prim. Trav. Sta. No. 72, 1916" ....... T. 23 N., R. 10 E., cormer of secs. $5,6,7$, and 8 .

T. 23 N., R. 10 E., SW. 各 sec. 4 , road fork.

Road forks

T. 23 N., R. 10 E., in south part of sec. 2,7 feet southwest of road where road bends from southeast to east, in large rock flush with ground; aluminum tablet stamped "Prim. Trav. Sta. No. 73, 1916"'.

T. $23 \mathrm{~N}$, , $\mathbf{R}$. i1 $\mathbf{E} ., 20$ feet west of corner fence post at second-class road south, quarter corner between secs. 7 and 8.

T. 23 N., R. 11 E., quarter corner between secs. 8 and 9,125 feet west of gate in east and west fence.

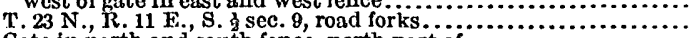

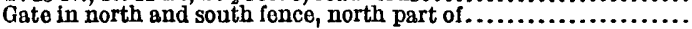

362839.6

362854.1

362934.6

362949.5

362958.9

362952.3

362941.0

$\begin{array}{lll}36 & 29 & 29.7\end{array}$

$\begin{array}{ll}36 & 29 \\ 39.6\end{array}$

$\begin{array}{ll}36 & 29 \\ 39.2\end{array}$

$\begin{array}{lll}36 & 29 & 34.3\end{array}$

362904.4

362904.7

$\begin{array}{lll}36 & 28 & 48.2\end{array}$

$\begin{array}{lll}36 & 28 & 36.7\end{array}$

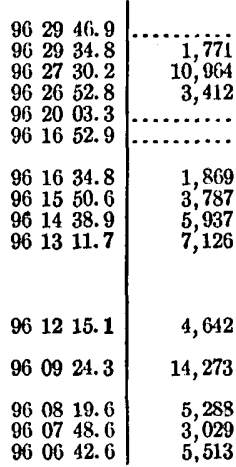


HOMINY QUADRATGLE-Continued.

Along north border of quadrangle-Continued.

\begin{tabular}{|c|c|c|c|}
\hline Station. & Latitude. & Longitude. & $\begin{array}{l}\text { Distance } \\
\text { between } \\
\text { stations. }\end{array}$ \\
\hline 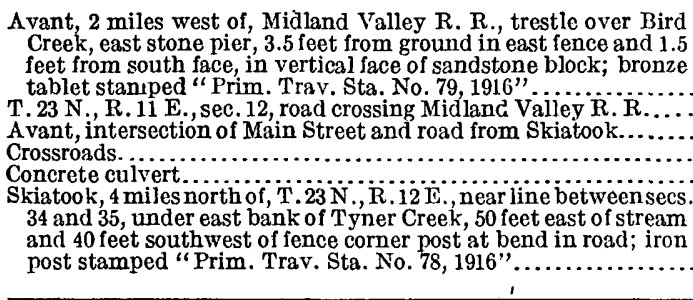 & $\begin{array}{ccc}\circ & , & \text { : } \\
30 & 29 & 03.0 \\
36 & 29 & 00.4 \\
36 & 29 & 16.2 \\
36 & 29 & 31.1 \\
36 & 29 & 39.4\end{array}$ & $\begin{array}{ccc}\circ & \prime & \prime \prime \\
96 & 05 & 15.6 \\
96 & 04 & 20.0 \\
96 & 03 & 41.7 \\
96 & 03 & 12.2 \\
96 & 02 & 05.4\end{array}$ & $\begin{array}{r}\text { Feet. } \\
7,5 S 9 \\
4,541 \\
3,518 \\
2,838 \\
5,522\end{array}$ \\
\hline
\end{tabular}

East across north half of quadrangle from west border to Skiatook. (By B. H. Yoakum and W. F. Hicks in 1916.)

T. 22 N., R. 8 E., corner of secs. $9,10,15$, and 16

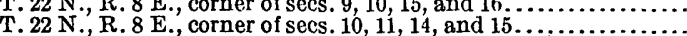
T. 22 N., R. 8 E., corner of secs. 11, 12, 13, and 14, just east of T road

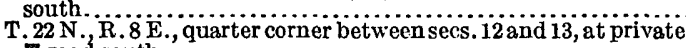
$T$ road south.

T. 22 N., Rs. 8 and 9 E., corner of secs. $7,12,13$, and 18 , at $T$ road south, 985 feet east of crossing of Missouri, Kansas \& Texas

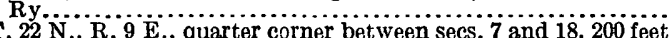
northeast of R. M. Miller's residence secs. 7 and 18, 200 leet T. 22 N., R. 9 E., corner of secs. $7,8,17$, and 18,22 feet north by 38 feet west of, in east side of north and south road and north side of T road east, 2 feet from fence corner ;iron post stamped "Prim. Trav. Sta. No. 70, 1916".

T. 22 N., R. 9 E., quarter corner between secs. 8 and 17 , center of east and west road at gate in ncrth and south fence line....... T. 22 N., R. 9 E., corner of secs. $8,9,16$, and 17 , center of east-west road at gate in north-south fence line...

T. 22 N., R. 9 E., quarter cormer between secs. 9 and 16,300 feet T. 22 N., R. $9 \mathrm{E}$., corner of secs. $9,10,15$, and 16 , at T road north.... T. 22 N., R. 9 E., in sec. 15 , forks of road............ T.22 N., R. 9 E., quarter corner between secs. 14 and 15,150 feet Boar Creek schoolhouse, 3,000 feet northwest of forks of road .......

T. 22 N., R. 9 E., corner of secs. 13, 14, 23, and 24, at Boar Creek schoolhouse, on hill at bend in road...

$T .22$ N., R. 9 E., quarter corner between secs. 13 and 24 , at fence line south.

T. 22 N., Rs. 9 and 10 E., corner of secs. $13,18,19$, and 24 , in center

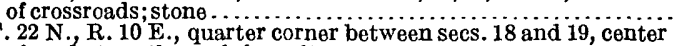
T. $22 \mathrm{~N}$., R. $10 \mathrm{E}$., quarter corner between secs. 18 and 19 , center T. 22 N., R. 10 E., corner of secs. $17,18,19$, and 20 , 110 feet southwest of road forks....

T. 22 N., R. $10 \mathrm{E}$., quarter corner between secs. 17 and 20,650 feet west of north and south road at $T$ fence east.

T. 22 N., R. $10 \mathrm{E}$., corner of secs. $15,16,21$, and 22, iron post stamped “Prim. Trav. Sta. No. 74, 1916. Elev. 682" ........ T. 22 N., R. 10 E., near quarter corner between secs. 15 and 22 , T. 22 N., $R .10 \mathrm{E} .$, near corner of secs. $13,14,23$, and 24, east part of gate, witness tree; nail in root of..

T. 22 N., Rs. 10 and 11 E., quarter corner between seos. 19 and 24

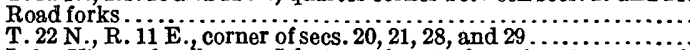

Lake View schoolhouse, 5 feet southeast of southeast corner of school yard;iron post stamped "Prim. Trav. Sta. No. 75, 1916".

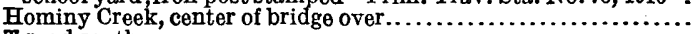

$T$ road south.

Quapaw Creek, bridge over; name plate at southwest corner........ T. 22 N., R. 12 E., corner of secs. $19,20,29$, and $30 \ldots$

T. 22 N., R. 12 E., quarter corner between secs. 20 and $21 . . . . . . .$. Crossroads.

Skiatook water tower, on no...................................... 3tamped "Prim. Trav. Sta. No. 76, 1916".

\begin{tabular}{|c|c|c|}
\hline $\begin{array}{lll}36 & 23 & 27.1 \\
36 & 23 & 25.8\end{array}$ & $\begin{array}{lll}96 & 26 & 33.1 \\
96 & 25 & 29.1\end{array}$ & $\ddot{5}, 240$ \\
\hline $\begin{array}{lll}36 & 23 & 26.1\end{array}$ & 962424.7 & \\
\hline $\begin{array}{lll}36 & 23 & 25.9\end{array}$ & $\begin{array}{lll}96 & 23 & 53.1\end{array}$ & \\
\hline $\begin{array}{lll}36 & 23 & 25.8\end{array}$ & 962321.5 & \\
\hline 362326.1 & 962249.3 & \\
\hline 362326.5 & 962216.6 & 2,68 \\
\hline $\begin{array}{lll}36 & 23 & 26.1\end{array}$ & $\begin{array}{lll}96 & 21 & 44.8\end{array}$ & \\
\hline $\begin{array}{lll}36 & 23 & 26.0\end{array}$ & 962112.4 & 2,6 \\
\hline $\begin{array}{lll}36 & 23 & 25.8 \\
36 & 23 & 25.6 \\
36 & 23 & 16.3\end{array}$ & $\begin{array}{lll}96 & 20 & 40.2 \\
96 & 20 & 08.1 \\
96 & 19 & 40.2\end{array}$ & $\begin{array}{l}2,634 \\
2,629 \\
2,463\end{array}$ \\
\hline $\begin{array}{lll}36 & 22 & 59.7 \\
36 & 22 & 53.5\end{array}$ & $\begin{array}{llll}96 & 19 & 03.9 \\
96 & 18 & 22.9\end{array}$ & $\begin{array}{l}3,41 \\
3,40\end{array}$ \\
\hline $\begin{array}{lll}36 & 22 & 33.5\end{array}$ & $\begin{array}{lll}96 & 17 & 59.1\end{array}$ & \\
\hline $\begin{array}{lll}36 & 22 & 33.4\end{array}$ & 961727.0 & \\
\hline 362233.5 & $\begin{array}{llll}96 & 16 & 54.7\end{array}$ & 2,6 \\
\hline $\begin{array}{lll}36 & 22 & 32.3\end{array}$ & $96 \quad 16 \quad 23.4$ & 2,568 \\
\hline $\begin{array}{lll}36 & 22 & 32.1\end{array}$ & 961551.2 & 2,63 \\
\hline $\begin{array}{lll}36 & 22 & 31.8\end{array}$ & $96 \quad 1519.1$. & 2,62 \\
\hline 362231.0 & $96 \quad 13 \quad 42.5$ & 7,906 \\
\hline $\begin{array}{lll}36 & 22 & 05.6\end{array}$ & $96 \quad 1234.8$ & 6,109 \\
\hline $\begin{array}{lll}36 & 22 & 31.0 \\
36 & 22 & 05.5 \\
36 & 22 & 04.5 \\
36 & 21 & 40.6\end{array}$ & $\begin{array}{lll}96 & 11 & 32.4 \\
96 & 10 & 27.7 \\
96 & 09 & 17.3 \\
96 & 08 & 18.7\end{array}$ & $\begin{array}{l}5,708 \\
5,890 \\
5,755 \\
5,369\end{array}$ \\
\hline $\begin{array}{lll}36 & 21 & 14.6 \\
36 & 21 & 00.2 \\
36 & 21 & 40.7 \\
36 & 21 & 40.9 \\
36 & 21 & 40.8 \\
36 & 22 & 06.4 \\
36 & 22 & 06.5\end{array}$ & $\begin{array}{lll}96 & 07 & 45.6 \\
96 & 06 & 42.6 \\
96 & 04 & 42.8 \\
96 & 04 & 07.9 \\
96 & 02 & 56.9 \\
96 & 01 & 52.2 \\
96 & 00 & 47.4\end{array}$ & $\begin{array}{r}3,773 \\
5,358 \\
10,625 \\
2,854 \\
5,809 \\
5,890 \\
5,300\end{array}$ \\
\hline $\begin{array}{lll}36 & 22 & 08.6\end{array}$ & $\begin{array}{llll}96 & 00 & 01.8\end{array}$ & 3,73 \\
\hline
\end{tabular}


HOMTNY QUADRANGLE-Continued.

Along east border of quadrangle.

\begin{tabular}{|c|c|c|c|}
\hline Station. & Latitude. & Longitude. & $\begin{array}{l}\text { Distance } \\
\text { betwren } \\
\text { stitions. }\end{array}$ \\
\hline 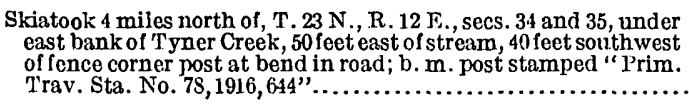 & $\begin{array}{ccc}\circ & \prime & \prime \prime \\
36 & 25 & 24.42\end{array}$ & $\stackrel{\circ}{\circ} \quad{ }_{06}^{\prime \prime} 03.86$ & $\begin{array}{c}\text { Feet. } \\
\ldots . . . .\end{array}$ \\
\hline
\end{tabular}

Magnetic declination for southeast quarter of quadrangle, $9^{\circ} \mathrm{E}$; for southwest quarter of quadrangle, $9^{\circ} 30^{\prime} \mathrm{E}$.; for northwest quarter of quadrangle, $9^{\circ} 40^{\prime} \mathrm{E}$.; for northeast quarter of quadrangle, $9^{\circ} 15^{\prime} \mathrm{E}$.

\section{KAW QUADRANGLE.}

West along highways near south border of quadrangle. (By B. H. Yoakum in 1916.)

\begin{tabular}{|c|c|c|c|}
\hline Station. & Latitude. & Longitude. & $\begin{array}{l}\text { Distance } \\
\text { between } \\
\text { stations. }\end{array}$ \\
\hline & 0,11 & - 11 & Fect. \\
\hline Station $353=$ Station 2, Book H 3376 , page $2 . \ldots \ldots \ldots \ldots \ldots \ldots \ldots \ldots$ & 364435.46 & 964541.77 & \\
\hline Milepost 21 , center of track opposite........................... & 364505.6 & 964617.0 & 4,190 \\
\hline 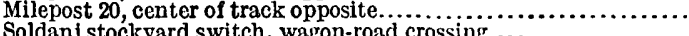 & 364530.1 & 964710.8 & $5,02 !$ \\
\hline $\begin{array}{l}\text { oldan1 stockyard switch, wagon-road crossing } \ldots \ldots \ldots \ldots \ldots \ldots \\
\text { Kaw, } 1.7 \text { miles southwest of, } 1,480 \text { feet west of road crossing at }\end{array}$ & 364532.3 & 964751.2 & 3,290 \\
\hline $\begin{array}{l}\text { Soldani stockyard switch, } 500 \text { feet east of milepost } 19 \text {, on top of } \\
\text { ridge, on north side of tracks near fence line; iron post stamped }\end{array}$ & & & \\
\hline 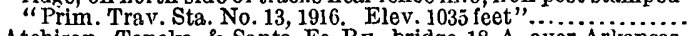 & 364538.7 & $96 \quad 48 \quad 07.4$ & 1,470 \\
\hline $\begin{array}{l}\text { Atchison, Topeka \& Santa Fe Ry. bridge } 18 \mathrm{~A} \text {, over } \mathrm{Arkansas} \\
\text { River, west end... }\end{array}$ & 3645423 & 0649150 & 5583 \\
\hline Kaw station, Atchison, Topeka \& Santa Fe Ry., center of track & & 864915.9 & 5,583 \\
\hline $\begin{array}{l}\text { opposite semaphore. } \\
\text { T. } 26 \text { N., R. } 4 \text { E., corner of secs. } 1,2,11 \text {, and } 12, T \text { road west......... } \\
\text { T. } 26 \text { N., R. } 4 \text { E., corner of secs. } 2,3,10 \text {, and } 11, \text { T road north.... } \\
\text { T. } 26 \text { N., R. } 4 \text { E., quarter corner between secs. } 3 \text { and } 10 \text {, on fence }\end{array}$ & 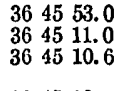 & $\begin{array}{l}96 \quad 4955.9 \\
9650 \quad 04.0 \\
965108.6\end{array}$ & $\begin{array}{l}3,433 \\
4,305 \\
5,256\end{array}$ \\
\hline 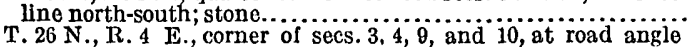 & 364510.4 & 865141.2 & 2,650 \\
\hline 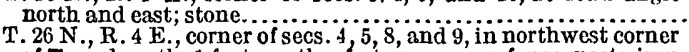 & 364510.1 & 865213.5 & 2,631 \\
\hline $\begin{array}{l}\text { of T road north, } 1 \text { foot north of stone corner fence post; iron } \\
\text { post stamped "Prim. Trav. Sta. No. 14, 1916". }\end{array}$ & 364510.0 & 965317.5 & 5,205 \\
\hline 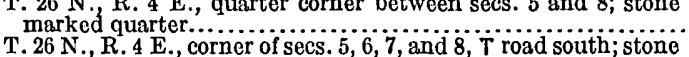 & 364510.0 & 965349.8 & 2,628 \\
\hline T. 26 N., Rs. 3 and 4 E., corner of secs. $1,6,7$, and i2, crossroads; & 364510.0 & 965421.8 & 2,610 \\
\hline $\begin{array}{l}\text { stone corner. } \\
\text { T. } 26 \text { N., R. } 3 \text { E., corner of secs.1, } 2,11 \text {, and } 12, \ldots \ldots \text {, center of crossroads. } \\
\text { T. } 26 \text { N., R. } 3 \text { E., corner or secs. } 2,3,10 \text {, and } 11 \text {, crossroads at school- }\end{array}$ & 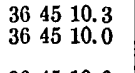 & 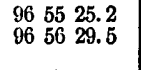 & $\begin{array}{l}5,153 \\
5,237\end{array}$ \\
\hline 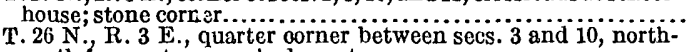 & 364510.0 & 965734.4 & 5,275 \\
\hline 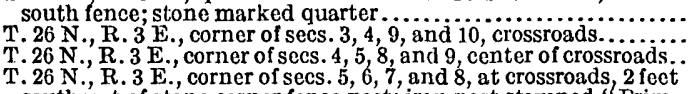 & 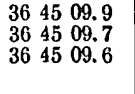 & $\begin{array}{l}965806.5 \\
965839.2 \\
965944.0\end{array}$ & $\begin{array}{l}2,618 \\
2,660 \\
5,270\end{array}$ \\
\hline 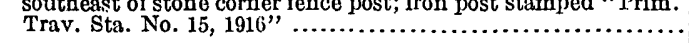 & $30 \quad 4509.2$ & 970048.0 & 5,209 \\
\hline
\end{tabular}

Magnetic declination along south border of quadrangle, $10^{\circ} 20^{\prime} \mathrm{E}$. 
RAW QUADRANGLE-Continued.

West along highways through center of quadrangle.

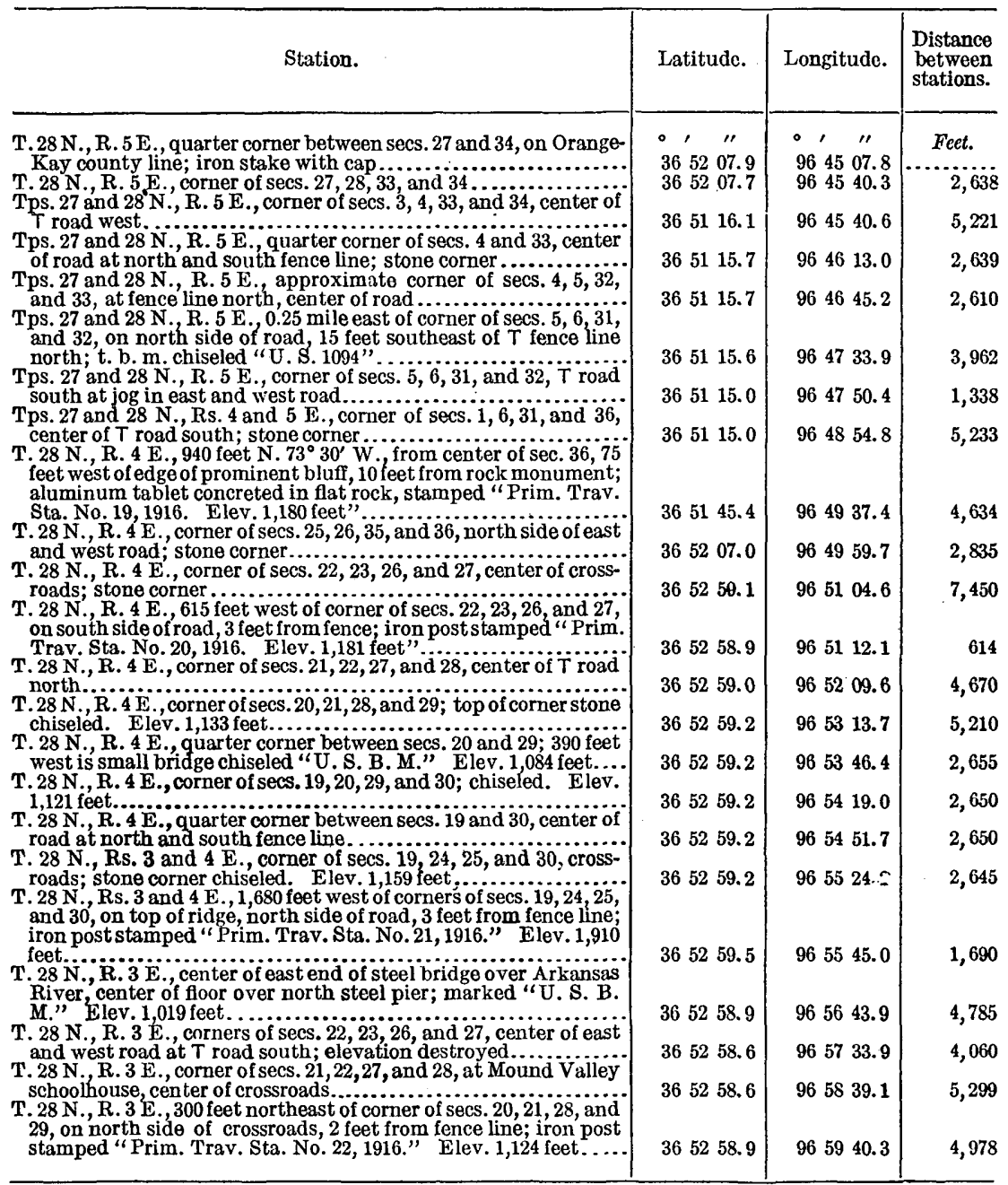

Magnetic declination through center of quadrangle (east-west), $10^{\circ} 22^{\prime} \mathrm{E}$.

East along highways near north border of quadrangle.

T. 29 N., R. 3 E., north corner of secs. 16 and $17, T$ road south.... Kansas-Oklahoma State line, milepost 109

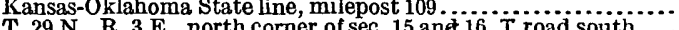
T. 29 N., R. 3 E., north corner of sec. 15 ane 16 , T road south..... Kansas-Oklahoma State line, milepost 108

T. 29 N., R. 3 E., north corner of secs. 14 and $15, T$ road south..... T. 35 S., R. 4 E., south corner of secs. 13 and 14,'T road north..... Kansas-Oklahoma State line, milepost 107 .................... T. 29 N., R. 3 E., north corner of secs. 13 and $14, T$ road south.... T. 35 S., R. 5 E., quarter corner of sec. 17, T road north on KansasOklahoma Stateline.

T. 29 N., R. 4 E., north corner of secs. 16 and 17 , at T road south, 1 foot south of corner stone on Kansas-Oklahoma State line; iron post stamped "Prim. Trav. Sta. No. 18, 1916"................ T. 29 N., R. 4 E., north corner of secs. 15 and 16 , T road south; stone corner....

T. 29 N., R. 4 E., north corner of secs. 14 and 15 , $T$ road south; T. $35 \mathrm{~S}$., R. 5 E., south corner of secs. 13 and $14, \mathrm{~T}$ road north.......

\begin{tabular}{lll|rrr|r}
36 & 59 & 55.7 & 96 & 59 & 36.1 & \\
36 & 59 & 55.9 & 96 & 58 & 52.8 & 3,510 \\
36 & 59 & 55.9 & 96 & 58 & 31.0 & 1,765 \\
36 & 59 & 55.9 & 96 & 58 & 02.2 & 2,340 \\
36 & 59 & 55.8 & 96 & 57 & 46.7 & 1,260 \\
36 & 59 & 55.8 & 96 & 57 & 26.2 & 1,660 \\
36 & 59 & 55.9 & 96 & 56 & 57.1 & 2,365 \\
36 & 59 & 56.0 & 96 & 56 & 41.7 & 1,250 \\
36 & 59 & 55.7 & 96 & 56 & 21.2 & 1,660 \\
36 & 59 & 56.2 & 96 & 54 & 12.7 & 10,429 \\
& & & & \\
36 & 59 & 56.3 & 96 & 53 & 07.9 & 5,252 \\
& & & & & \\
36 & 59 & 56.5 & 96 & 52 & 02.5 & 5,305 \\
& & & & & \\
36 & 59 & 56.4 & 96 & 50 & 57.2 & 5,300 \\
36 & 59 & 56.4 & 96 & 50 & 26.7 & 2,475
\end{tabular}

365955.7 365955.9 365955.9 3659

365956.0 $\begin{array}{lll}36 & 59 & 55.7\end{array}$

365956.2

$\begin{array}{lll}36 & 59 & 56.3\end{array}$

$\begin{array}{lll}36 & 5956.4\end{array}$ 965026.7
638

639

, 610

962

338

33

634

614

655

650

650

645

690

785

4,060

, 299

978 
KAW QUADRANGLE-Continued.

Erst along highways near north border of quadrangle-Continued.

\begin{tabular}{|c|c|c|c|}
\hline Station. & Latitude. & Longitude. & $\begin{array}{l}\text { Distance } \\
\text { between } \\
\text { stations. }\end{array}$ \\
\hline 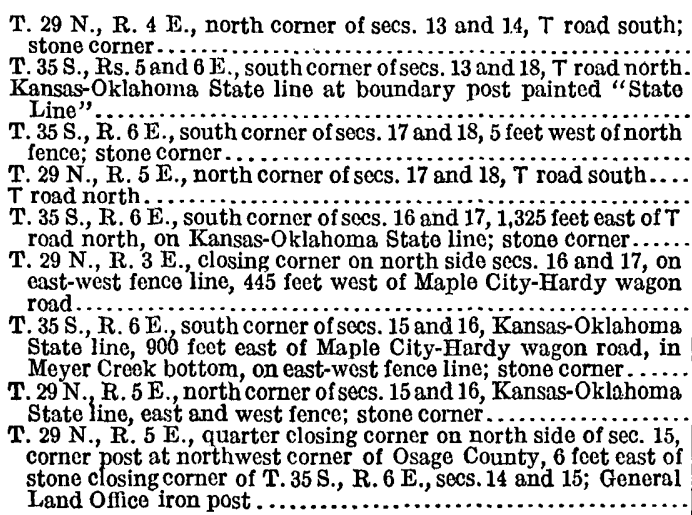 & $\begin{array}{ccc}\circ & \prime & \prime \prime \\
36 & 59 & 56.4 \\
36 & 59 & 56.4 \\
36 & & \\
39 & 56.3\end{array}$ & $\begin{array}{ccc}\circ & \prime & \prime \prime \\
96 & 49 & 52.0 \\
96 & 49 & 21.4 \\
96 & 49 & 00.4 \\
& & \\
96 & 48 & 15.9 \\
96 & 47 & 43.0 \\
96 & 47 & 27.1 \\
96 & 47 & 10.8\end{array}$ & $\begin{array}{r}\text { Feet. } \\
2,820 \\
2,480 \\
1,703\end{array}$ \\
\hline
\end{tabular}

Magnetic declination along north border of quadrangle, $10^{\circ} 10^{\prime} \mathrm{E}$.

South along highways near south border of quadrangle.

Tps. 26 and 27 N., R. 5 E., quarter corner between secs. 3 and 34; Tps. 26 and 27 N., R. 5 E., corner of secs. $3,4,33$, and 34 ; iron stake

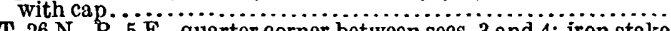
T. 26 N., R. 5 E., quarter corner between secs. 3 and 4 ; iron stake T. 26 N., R. 5 E. corner of secs. $3,4,9$, and 10 ; iron stake with cap..

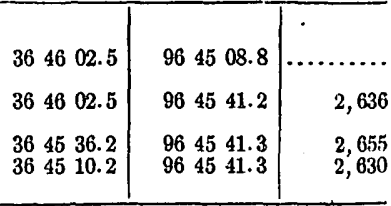

MALLON QUADRANGLE.

Along highway in northwest corner of quadrangle. (By B. H. Yo\&kum in 1916.)

\begin{tabular}{|c|c|c|c|}
\hline Station. & Latitude. & Longitude. & $\begin{array}{l}\text { Distance } \\
\text { between } \\
\text { stations. }\end{array}$ \\
\hline 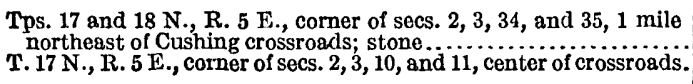 & $\begin{array}{ccc}\circ & \prime & \prime \prime \\
35 & 59 & 08.1 \\
35 & 58 & 15.8\end{array}$ & $\begin{array}{ccc}\circ & \prime \prime \\
96 & 44 & 58.8 \\
96 & 44 & 57.5\end{array}$ & $\begin{array}{l}\text { Feet. } \\
-3,285\end{array}$ \\
\hline
\end{tabular}

Magnetic declination for extreme northwest corner of quadrangle, $9^{\circ} 18^{\prime} \mathrm{E}$.

South along highways through center of quadrangle. (By B. H. Yoakum in 1916.)

Drumright, intersection of Skinner Avenue and Broadway Street, quarter corner between secs. 6 and 31 , Tps. 17 and 18 N., R. $7 \mathrm{E}$. Drumright, 1.5 miles southwest of, quarter corner between secs. 6 and 7, T. 17 N., R. 7 E., at crossroads; General Land Offico iron

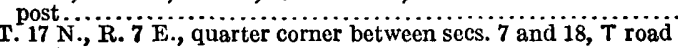
T. 17 N., $\dddot{R} .7$. $\mathrm{E}$. , quarter corner between sces. 18 and 19 , crossroads.

T. 17 N., $R$. 7 E., corner of secs. $19,20,29$, and $30 \ldots \ldots, \ldots \ldots \ldots$ General Land Office iron post at $T$ road north ................. $T$. 17 N., R. 7 E., west corner of secs. 30 and $3 i, T$ road east on

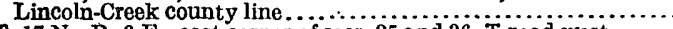

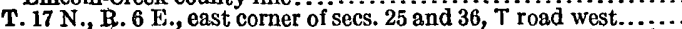
T. 17 N., Rs. 6 and 7 I., south corner of sees. 31 and 36 , on LincolnCreek county line, at angle in road; iron post stamped "Prim. Trav. Sta. No. 39, 1916. Elev. 977',

\begin{tabular}{|c|c|c|}
\hline $\begin{array}{lll}35 & 59 & 18.6\end{array}$ & $\begin{array}{lll}96 & 36 & 37.8\end{array}$ & \\
\hline $\begin{array}{lll}35 & 58 & 25.7\end{array}$ & 963638.5 & 5,353 \\
\hline $\begin{array}{lll}35 & 57 & 33.3 \\
35 & 56 & 41.4 \\
35 & 55 & 48.6\end{array}$ & $\begin{array}{llll}96 & 36 & 38.2 \\
96 & 36 & 38.5 \\
96 & 36 & 05.8\end{array}$ & $\begin{array}{l}5,302 \\
5,246 \\
5,281\end{array}$ \\
\hline 355456.6 & $\begin{array}{llll}96 & 36 & 06.1\end{array}$ & 5,257 \\
\hline $\begin{array}{lll}35 & 54 & 56.9 \\
35 & 54 & 47.0\end{array}$ & $\begin{array}{lll}96 & 37 & 11.0 \\
96 & 37 & 11.1\end{array}$ & $\begin{array}{l}5,337 \\
1,002\end{array}$ \\
\hline $\begin{array}{llll}35 & 54 & 04.5\end{array}$ & 963711.0 & 1,293 \\
\hline
\end{tabular}


MALION QUADRANGLE-Continued.

Along highways south through center of triangle-Continued.

\begin{tabular}{|c|c|c|c|}
\hline Station. & Latitude. & Longitude. & $\begin{array}{l}\text { Distance } \\
\text { between } \\
\text { stations. }\end{array}$ \\
\hline 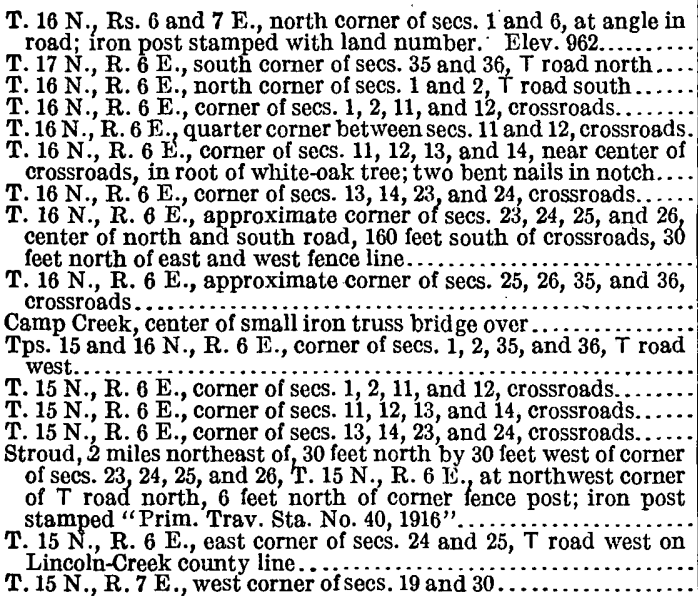 & $\begin{array}{cccc}\circ & \prime & & \prime \prime \\
35 & 54 & 04.5 \\
35 & 53 & 54.5 \\
35 & 53 & 54.8 \\
35 & 53 & 03.8 \\
35 & 52 & 37.9 \\
35 & 52 & 11.8 \\
35 & 51 & 19.3 \\
& & & \\
35 & 50 & 26.8 \\
& & \\
35 & 49 & 34.4 \\
35 & 49 & 01.1 \\
& & & \\
35 & 48 & 42.6 \\
35 & 47 & 49.4 \\
35 & 46 & 56.9 \\
35 & 46 & 04.6 \\
& & & \\
& & & \\
35 & 45 & 13.0 \\
35 & 45 & 12.9 \\
35 & 45 & 18.8\end{array}$ & $\begin{array}{cccc}\circ & \prime & \prime \prime \\
96 & 37 & 07.3 \\
96 & 37 & 27.8 \\
96 & 37 & 40.7 \\
96 & 37 & 40.6 \\
96 & 37 & 40.4 \\
& & \\
96 & 37 & 40.1 \\
96 & 37 & 39.5 \\
& & \\
96 & 37 & 39.0 \\
& & \\
96 & 37 & 38.9 \\
96 & 37 & 38.3 \\
& & \\
96 & 37 & 38.4 \\
96 & 37 & 37.6 \\
96 & 37 & 37.2 \\
96 & 37 & 37.0 \\
& & \\
& & \\
96 & 37 & 37.4 \\
96 & & \\
96 & 11.3 \\
96 & 37 & 10.8\end{array}$ & $\begin{array}{r}\text { Feet. } \\
296 \\
1,966 \\
1,060 \\
5,154 \\
2,625 \\
2,638 \\
5,305 \\
\\
5,310\end{array}$ \\
\hline
\end{tabular}

East along highways near south border of quadrangle.

T.15 N., R. 7 E., corner of secs. $19,20,29$, and 30 , crossroads .... T. 15 N., R. 7 E., corner of secs. $20,21,28$, and 29 , T road south, in line with east and west road at road northwest.

Camp Creek, east end of approach to bridge.

Milfay, crossroads, stone corner of secs. $21,22,27$, and $2 \ddot{2}, \ddot{T} . \ddot{15} \mathrm{~N}$.,

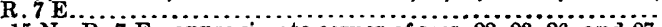
T. 15 N, $\mathrm{R} .7 \mathrm{E}$, approximate corner of secs, $22,23,26$, and 27 , $T$ road west, 70 feet south of road forks.

T. 15 N., R. $7 \mathrm{E}$., corner of secs. $23,24,25$, and 26 , road forks..... T. 15 N., Rs. 7 and 8 E. corner of secs. $19,24,25$, and 30 , crossroads T. 15 N., Rs. 7 and $8 \mathrm{E}$., corner of secs. $13,18,19$, and 24,19 feet northeast of bearing tree marked "S24", 15 feet northwest of bearing tree marked "S19"; chiseled square in sandstone..

T. 15 N., Rs. 7 and 8 E., 30 feet south by 25 feet east of corner of secs. 7, 12,13, and 18, at corner of wire fence, in southeast corner of crossroads; iron post stamped "Prim. Trav. Sta. No.7, 1914".

\begin{tabular}{lll|lll|l}
35 & 45 & 18.6 & 96 & 36 & 03.7 & 5,535 \\
35 & 45 & 18.7 & 96 & 34 & 59.7 & 5,274 \\
35 & 45 & 19.0 & 96 & 34 & 27.3 & 2,670 \\
& & & & & \\
35 & 45 & 18.7 & 96 & 33 & 55.7 & 2,604 \\
& & & & & & \\
35 & 45 & 18.9 & 96 & 32 & 51.8 & 5,265 \\
35 & 45 & 19.0 & 96 & 31 & 47.8 & 5,276 \\
35 & 45 & 19.1 & 96 & 30 & 43.7 & 5,282 \\
& & & & & \\
35 & 46 & 11.4 & 96 & 30 & 43.6 & 5,282 \\
& & & & & \\
35 & 47 & 03.4 & 96 & 30 & 43.3 & 5,262
\end{tabular}

East along highways through center of quadrangle.

Shamrock, 0.5 mile northwest of, near quarter corner of secs. 29 and $32, \mathrm{~T}, 17 \mathrm{~N}, \mathrm{R}, 7 \mathrm{~F}$

Shamrock, railroad crossing at station, main track T. 17 N., k. 7 E., quarter corner between secs. 27 and 34,70 feet west of crossroads, in top of ridge; iron pipe.

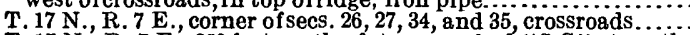

T. 17 N., R. 7 E., 850 feet north of stone marked " $\mathrm{S} C$ " at south

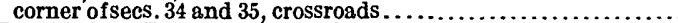
T. 17 N., R. $7 \mathrm{E}$., south corner ofsecs. 35 and 36,40 feet southeast of corner fence post; stone marked "S C".

T. $17 \mathrm{~N}$., Rs. 7 and $8 \mathrm{E}$., south corner of secs. 31 and 36 ; iron post stamped witt. General Land Office numbers. Elev. 949

T. $16 \mathrm{~N}$. Rs. 7 and $8 \mathrm{E}$. secs, 1 and 6 , north corner of; iron post stamped with General Land Office numbers, also elevation 958: "Prim. Trav. Sta. No. 5, 1914" (112 feet west of this station is standard iron post, stamped with Generol Land Office numbers for T. 17 N., Rs. 7 and 8 E., secs. 31 and 36 , north corner)......

\begin{tabular}{|c|c|c|}
\hline $\begin{array}{lll}35 & 54 & 56.7 \\
35 & 54 & 47.7\end{array}$ & $\begin{array}{lll}96 & 35 & 35.7 \\
96 & 34 & 26.6\end{array}$ & $\cdots \cdots$ \\
\hline $\begin{array}{lll}35 & 54 & 56.6 \\
35.54 & 56.7\end{array}$ & $\begin{array}{lll}96 & 33 & 25.3 \\
96 & 32 & 53.5\end{array}$ & $\begin{array}{l}5,123 \\
2,614\end{array}$ \\
\hline 355421.3 & $\begin{array}{lll}96 & 32 & 53.3\end{array}$ & 4,492 \\
\hline 355404.4 & 963148.8 & 5,365 \\
\hline 3554044 & 963045.6 & 5,201 \\
\hline $\begin{array}{lll}35 & 54 & 04.4\end{array}$ & 963043.0 & 21 \\
\hline
\end{tabular}

Magnetic declination north and south through center of quadrangle, $9^{\circ} 32^{\prime} \mathrm{E}$; east and west through center of quadrangle, $9^{\circ} 22^{\prime} \mathrm{E}$; f for south border of quadrangle, $10^{\circ} 07^{\prime} \mathrm{E}$. 
NEWEIRK QUADRANGLE.

North along highways near west border of quadrangle (north half). (By B. H. Yoakum in 1916).

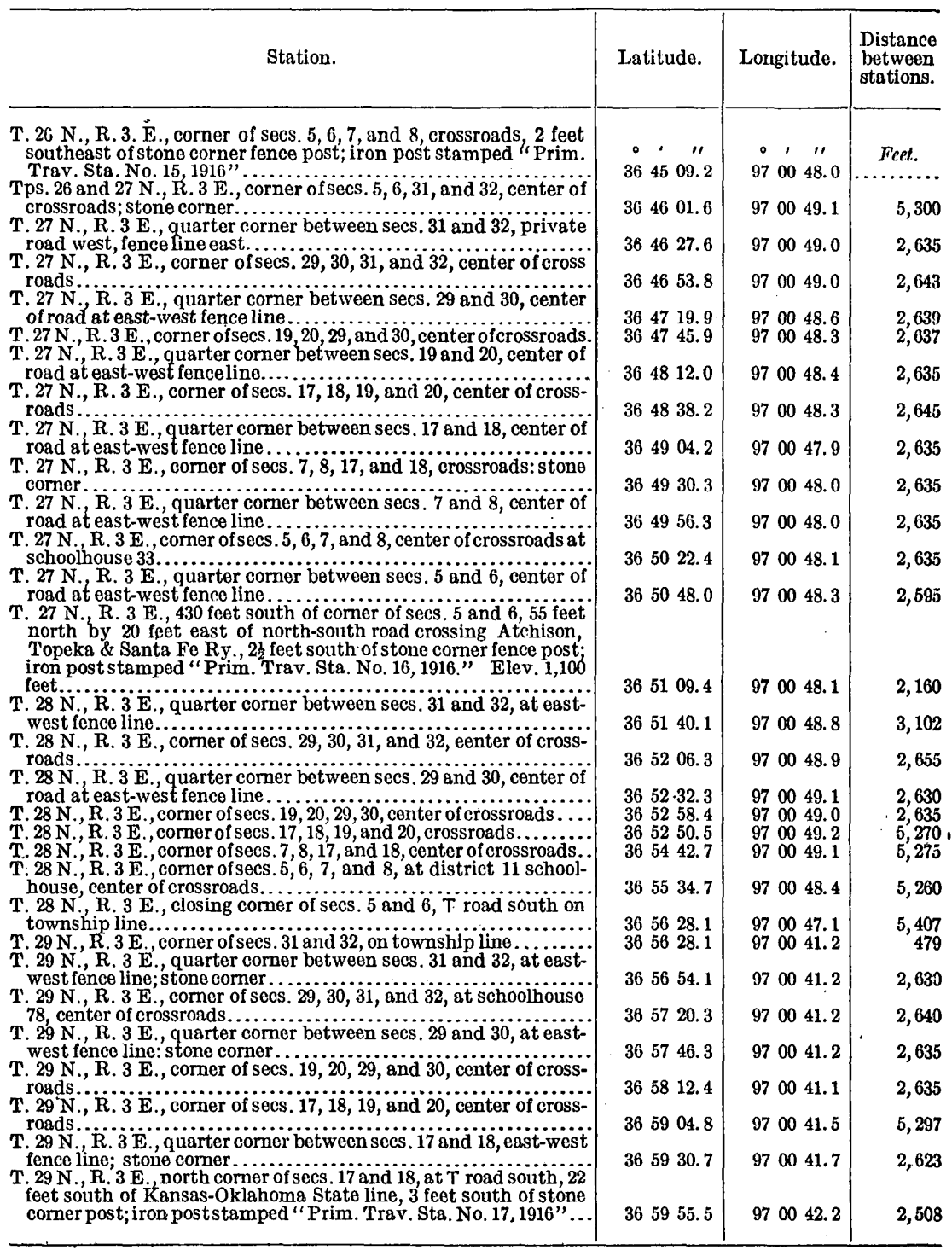

Magnetic declination along east border of quadrangle (north half), $10^{\circ} 04^{\prime} \mathrm{E}$. 
NEWKIRK QUADRANGLE-Continued.

Along highways near east border of quadrangle (south half).

\begin{tabular}{|c|c|c|c|}
\hline Station. & Iatitude. & Longitude. & $\begin{array}{l}\text { Distance } \\
\text { between } \\
\text { stations. }\end{array}$ \\
\hline 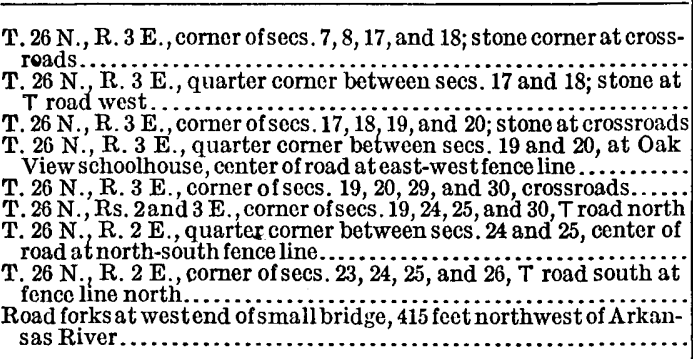 & \begin{tabular}{ccc|}
$\circ$ & $\prime$ & $\prime \prime$ \\
36 & 44 & 17.5 \\
36 & 43 & 51.6 \\
36 & 43 & 25.6 \\
& & \\
36 & 42 & 59.6 \\
36 & 42 & 33.8 \\
36 & 42 & 33.3 \\
36 & 42 & 33.1 \\
36 & 42 & 33.0
\end{tabular} & $\begin{array}{cccc}\circ & \prime \prime & \\
97 & 00 & 48.4 \\
& & \\
97 & 00 & 48.4 \\
97 & 00 & 48.4 \\
& & & \\
97 & 00 & 48.3 \\
97 & 00 & 48.2 \\
97 & 01 & 52.4 \\
97 & 02 & 24.5 \\
97 & 02 & 57.0\end{array}$ & $\begin{array}{r}\text { Feet. } \\
\ldots \ldots \ldots \\
2,627 \\
2,631 \\
\\
2,622 \\
2,614 \\
5,230 \\
2,615 \\
2,645\end{array}$ \\
\hline
\end{tabular}

Along highways near east border of quadrangle (north half).

T. 26 N., R. 2 E., southeast end of Arkansas River bridge, 1.75 miles southeast of Ponca City ..................................... quarter corner between secs. 35 and 36 , center of

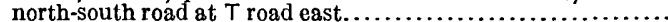

T. 26 N., R. 2 E., center of sec. 36, center of east-west road at north-

T. $26 \mathrm{~N}$., Rs. 2 and 3 E. quarter corner between secs. 36 and 31 , center of east-west road at fence line north $\ldots \ldots \ldots \ldots \ldots \ldots \ldots \ldots$

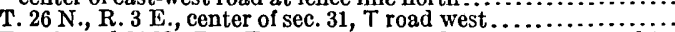
Tps. 25 and 26 N., R. 3 E., quarter corner between secs. 6 and 31 ,

Tps. 25 and 26 N., Rs. 2 and 3 E.. corner of secs. $1,6,31$. and 36 . center of east-west road at T road south, 26 feet west of $T$ fence line;

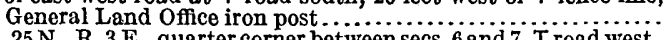

T. 25 N., R. 3 E., quarter corner between secs. 6 and $7, T$ road west.

T. 25 N., R. 3 E., center of sec. 7, T road east

T. 25 N., R. 3 E., quarter corner between secs. 7 and 8 ; iron stake.

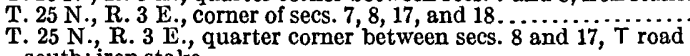
T. 25 N., R. 3 E., quarter corner between secs. 17 and 20 , center of

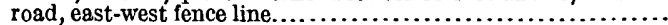

T. $25 \mathrm{~N}$., R. $3 \mathrm{E}$., center of sec. 20,15 feet north of $\mathrm{T}_{\mathrm{K}}$ road east, in line with fence east and west on west side of road, 13 feet from fence post; iron post stamped "Prim. Trav. Sta. No. 23, 1916".........

$T$ road west.

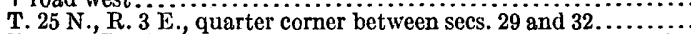
T. 25 N., R. 3 E., apparent corner of secs. 29, 30, 31, and 32; stone in center of east-west road at $T$ fence south.

T. 25 N., R. 3 E., north comer of secs. 5 and 6 , in Arkansas $R i v e r$ bottom, one-half mile north of $\Lambda$ rkansas River, 20 feet west by 25 feet south of end post of fence line east, General Land Office

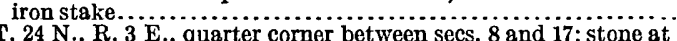
T. 24 N., R. 3 E. quarter corner between secs. 8 and 17; stone at

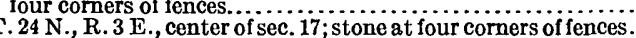

T. 24 N., R. 3 E., quarter comer between secs. 17 and 20 , at J. E. A lexander's residence, center of east-west road at fence line northsoutr.

T. 24 N., R. 3 E., quarter corner between secs. 20 and 29 ; stone at center of east and west road at fences north and south............
364134.9

$\begin{array}{lll}36 & 41 & 15.9\end{array}$

364115.8

$3641 \quad 15.5$

$\begin{array}{lll}36 & 41 & 15.6\end{array}$

$\begin{array}{lll}36 & 40 & 49.8\end{array}$

$36 \quad 40 \quad 49.6$

$\begin{array}{lll}36 & 39 & 57.8\end{array}$

$36 \quad 3932.6$

$\begin{array}{llll}36 & 39 & 32.2\end{array}$

363906.3

363906.0

363814.2

$\begin{array}{lll}36 & 37 & 47.8\end{array}$

363702.5

$\begin{array}{llll}36 & 36 & 29.7\end{array}$

363629.8

$\begin{array}{lll}36 & 35 & 37.7\end{array}$

$\begin{array}{lll}36 & 33 & 52.7\end{array}$

$\begin{array}{lll}36 & 33 & 26.9\end{array}$

363300.8

363208.6 $\begin{array}{lll}97 & 02 & 57.6\end{array}$

1,125

970257.0

$\begin{array}{lll}97 & 02 & 24.8\end{array}$

$\begin{array}{lll}97 & 01 & 52.1\end{array}$

970120.0

970120.2

$\begin{array}{lll}97 & 01 & 52.4\end{array}$

970120.3

970119.8

$9700 \quad 47.9$

$\begin{array}{lll}97 & 00 & 47.8\end{array}$

$\begin{array}{llll}97 & 00 & 15.4\end{array}$

970015.2

970015.4

970015.2

970015.5

970047.1

4,589

3,320

2,576

970052.7

5,285

$\begin{array}{lll}97 & 00 & 19.7\end{array}$

970019.0

2,610

970019.5

2,640

970019.5

5,279

Magnetic declination for east border of quadrangle, $10^{\circ} 30^{\prime} \mathrm{E}$., south half of SW. $\frac{1}{4}$ of quadrangle; for east border of quadrangle, $10^{\circ} 25^{\prime} \mathrm{E}$., north half of SW. $\frac{1}{4}$ of quadrangle.

WOWATA QUADRANGLE.

Near southwest corner of quadrangle. (By B. H. Yoakum in 1916.)

\begin{tabular}{|c|c|c|c|}
\hline Station. & Latitude. & Longitude. & $\begin{array}{l}\text { Distance } \\
\text { between } \\
\text { stations. }\end{array}$ \\
\hline T. 23 N., R. 12 E., near northeast corner of sec. 2, road corner. . . . . . . & $\begin{array}{ccc}\circ & , \prime \prime \\
36 & 30 & 24.7\end{array}$ & $\begin{array}{ccc}\circ & , \prime \prime \\
95 & 59 & 12.8\end{array}$ & Feet. \\
\hline
\end{tabular}


PAWHUSKA QUADRANGLE.

North along highways near west border of quadrangle. (By W. F. Hicks in 1916.)

\begin{tabular}{|c|c|c|c|}
\hline Station. & Latitude. & Iongitude. & $\begin{array}{l}\text { Distance } \\
\text { between } \\
\text { stations. }\end{array}$ \\
\hline 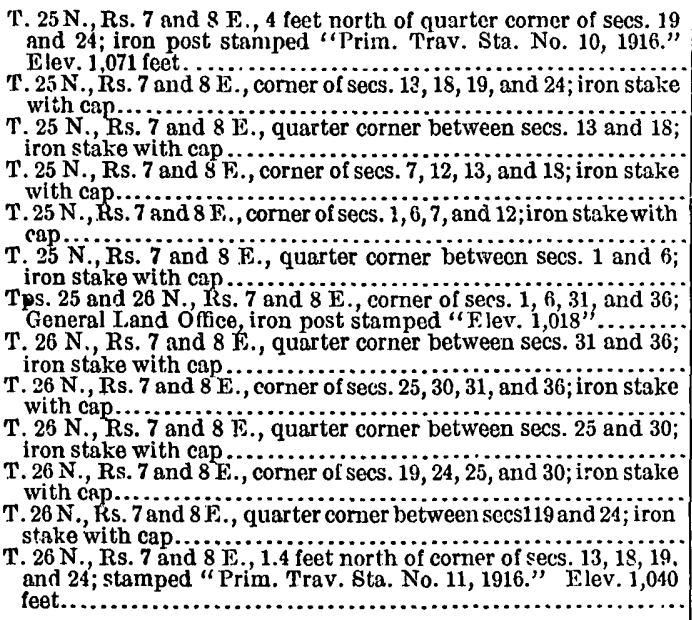 & $\begin{array}{ccc}\circ & \prime \prime & \prime \prime \\
36 & 37 & 47.1 \\
36 & 38 & 13.2 \\
36 & 38 & 39.4 \\
36 & 39 & 05.5 \\
36 & 39 & 57.9 \\
36 & 40 & 24.1 \\
36 & 40 & 50.2 \\
36 & 41 & 16.5 \\
36 & 41 & 42.6 \\
36 & 42 & 08.8\end{array}$ & $\begin{array}{ccc}\circ & \prime \prime & \prime \prime \\
96 & 29 & 35.3 \\
96 & 29 & 35.1 \\
96 & 29 & 34.6 \\
96 & 29 & 34.5\end{array}$ & $\begin{array}{l}2,648 \\
2,647 \\
2,653 \\
2,642\end{array}$ \\
\hline
\end{tabular}

East along south border of quadrangle. (By W. F. Hicks in 1910.)

T. 23 N., R. 8 E., near line between secs. 2 and 3,50 feet east of road fork, 150 feet west of north and south fence; nail in root of 15-inch hickory tree.

T. 23 N., $R .8$ E., in sec. 2 , road fork

Tps. 23 and 24 N., R. 8 E., comer of secs. $1,2,35$, and 36 .

Tps. 23 and 24 N, R. 8 E., quarter corner between secs. 1 and 36

Tps. 23 and 24 N., Rs. 8 and 9 F., at township corner, 20 feet west of intersection of crossroads...?

Tps. 23 and 24 N., R.9 E., in sec. $5, T$ road south from township line and along west side of Missouri, Kansas \& Texas Ry.

Tps. 23 and 24 N.,R. 9 E., at quarter corner between secs. 5 and 32 750 foet east of Missouri, Kansas \& Texas Ry.;iron post stamped

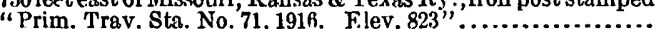
Center of road at section-line fence north and south

T. 23 N., P. 9 E., in sec. 2 , road forks..

Tps. 23 and $24 \mathrm{~N}, \mathrm{R}, 12 \mathrm{E}$, east closing corne of secs. 3 and 34 road, 25 feet west of north and south phone line; General Land Office post stamped " Prim. Trav. Sta. No. 77, 1916"

\begin{tabular}{|c|c|c|}
\hline $\begin{array}{llll}36 & 30 & 03 . & 6 \\
36 & 30 & 08.0 \\
36 & 30 & 16.0 \\
36 & 30 & 23 & 4 \\
36 & 30 & 23.4\end{array}$ & $\begin{array}{lll}96 & 25 & 32.0 \\
96 & 24 & 56.4 \\
96 & 24 & 43.9 \\
96 & 24 & 26.6 \\
96 & 23 & 54.4\end{array}$ & $\begin{array}{r} \\
2,945 \\
1,302 \\
1,601 \\
2,630\end{array}$ \\
\hline $\begin{array}{lll}36 & 30 & 23.4\end{array}$ & 962322.1 & 2,641 \\
\hline 363023.0 & 862154.0 & 7,192 \\
\hline $\begin{array}{lll}36 & 30 & 23.1 \\
36 & 50 & 21.8 \\
36 & 30 & 23.8\end{array}$ & $\begin{array}{llll}96 & 21 & 41.0 \\
96 & 21 & 10.4 \\
96 & 18 & 35 & 2\end{array}$ & $\begin{array}{r}821 \\
2,742 \\
12,675\end{array}$ \\
\hline 363023.6 & 9600.03 .3 & $\ldots \ldots \ldots$ \\
\hline
\end{tabular}

PRAGUE QUADRANGLE.

(By B. H. Yoakum in 1916.)

\begin{tabular}{|c|c|c|c|}
\hline Station. & Latitude. & Tongitude. & $\begin{array}{l}\text { Distanco } \\
\text { between } \\
\text { stations. }\end{array}$ \\
\hline $\begin{array}{l}\text { Paden, } 3 \text { miles east and } 0.7 \text { mile south of, } 957 \text { feet south of quarter } \\
\text { corner between secs. } 19 \text { and } 24, T \text {. } 12 \text { N.. Rs. } 7 \text { and } 8 \text { E., } 38 \text { feet } \\
\text { north by } 30 \text { feet east of Fort Smith \& Western } \mathrm{R} \text {. R. crossing, } \\
3 \text { feet southwest of corner fence post of railroad property line } \\
\text { fence; iron post stamped "Frim. Trav. Sta. No. } 44,1916 \text { "....... }\end{array}$ & $\begin{array}{ccc}\circ & \prime & \prime \prime \\
35 & 29 & 52.1\end{array}$ & $\begin{array}{ccc}\circ & \prime \prime & \prime \prime \\
96 & 30 & 42.0\end{array}$ & Feet. \\
\hline
\end{tabular}


SAC AND FOX QUADRANGLE.

Along highways south through center of quadrangle. (By B. H. Yoakum in 1916.)

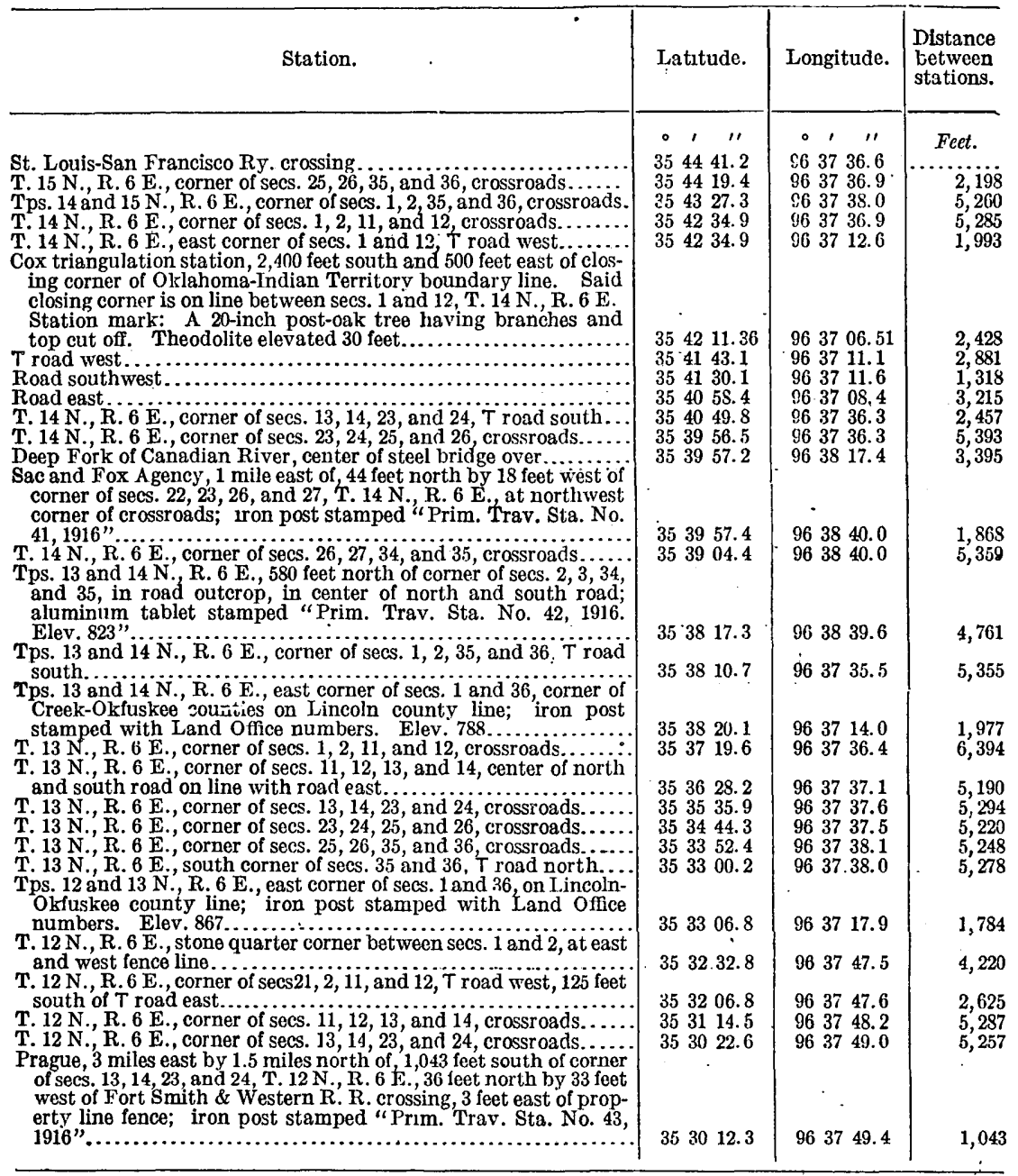

East along highways and Fort Smith \& Western R. R. near south border of quadrangle.

\begin{tabular}{|c|c|c|c|}
\hline $\begin{array}{l}\text { T. } 12 \text { N., R. } 7 \text { E., railroad crossing on line between secs. } 17 \text { and } 18 . \\
\text { T. } 12 \text { N., R. } 7 \text { E., railroad crossing, } 395 \text { feet west of corner of secs. }\end{array}$ & $\begin{array}{lll}35 & 3053.6\end{array}$ & 963601.5 & 9,852 \\
\hline 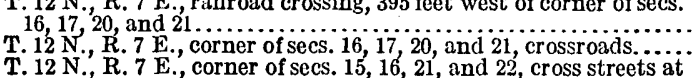 & $\begin{array}{lll}35 & 30 & 28.3 \\
35 & 30 & 28.3\end{array}$ & $\begin{array}{lll}96 & 35 & 02.5 \\
96 & 34 & 57.7\end{array}$ & $\begin{array}{r}5,508 \\
395\end{array}$ \\
\hline Baptist Church at Paden.................... & $\begin{array}{lll}35 & 30 & 28.3\end{array}$ & 9633 & 5,245 \\
\hline d troctlo 1503 a & & & 1,9 \\
\hline Railro & 353023. & $\begin{array}{l}250.2 \\
256.2\end{array}$ & 2,902 \\
\hline Railro & $\begin{array}{llll}35 & 30 & 17.2\end{array}$ & 237.7 & \\
\hline Railr & & & 2,997 \\
\hline T. 12 & $\begin{array}{llll}35 & 30 & 00.3\end{array}$ & $\begin{array}{lll}96 & 31 & 46.3\end{array}$ & 1,737 \\
\hline
\end{tabular}


SAC AND FOX QUADRANGLE-Continued.

North along highways near east border of quadrangle.

\begin{tabular}{|c|c|c|c|}
\hline Station. & Latitude. & Longitude. & $\begin{array}{l}\text { Distance } \\
\text { between } \\
\text { stations. }\end{array}$ \\
\hline 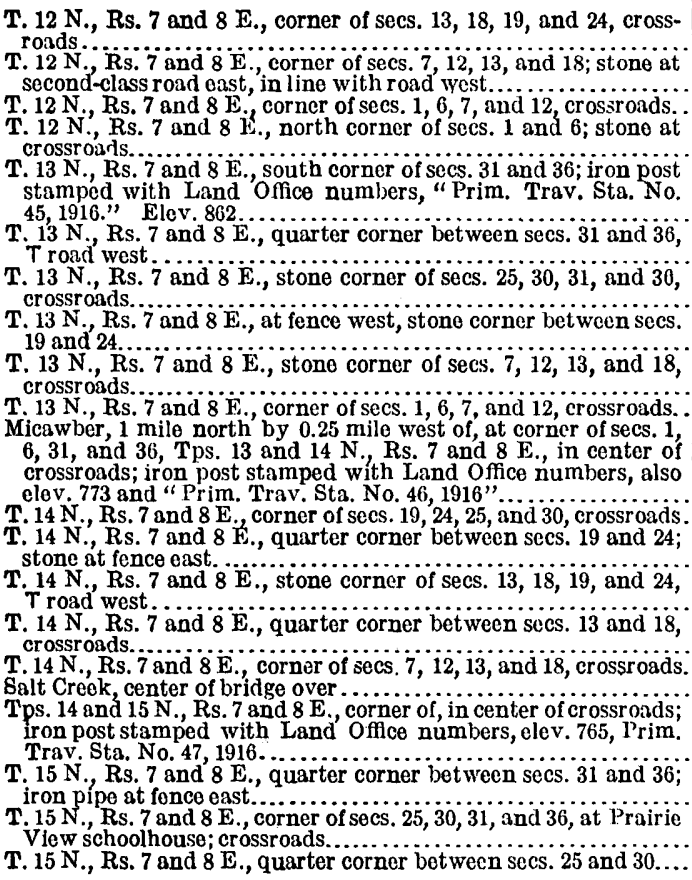 & $\begin{array}{ccc}\circ & \prime & \prime \prime \\
35 & 30 & 27.7 \\
35 & 31 & 20.0 \\
35 & 32 & 12.2 \\
35 & 33 & 07.3 \\
35 & 33 & 07.3 \\
35 & 33 & 33.4 \\
35 & 33 & 59.6 \\
35 & 35 & 17.8\end{array}$ & $\begin{array}{cccc}\circ & \prime & \prime \prime \\
96 & 30 & 42.4 \\
96 & 30 & 42.4 \\
96 & 30 & 42.4 \\
96 & 30 & 42.5\end{array}$ & $\begin{array}{c}\text { Feet. } \\
\ldots \ldots \ldots \\
5,286 \\
5,284 \\
5,566\end{array}$ \\
\hline
\end{tabular}

West along highways through center of quadrangle.

T. 13 N., R. 7 E., corner of secs, 1, 2, 11, and 12, center of east and west road at north and south fence line... T. 13 N., R. 7 E., quarter corner between secs. 2 and 11 ; stone at

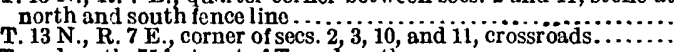

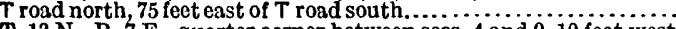

T. 13 N., R. 7 E., quarter corner between secs. 4 and 9 , 10 feet west T. 13 N., R. 7 E., stone corner of secs. $4,5,8$ and $9, \tau_{\text {T }}$ road south. T. $13 \mathrm{~N} .$, R. $7 \mathrm{E}$. , stone corner of secs. $4,5,8$, and 9 , T road south.
T. $13 \mathrm{~N} ., \mathrm{R} .7 \mathrm{E}$., stone quarter cormer between secs. 5 and $8, T$

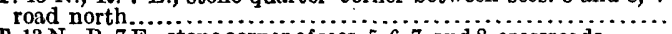
T. 13 N., R. 7 E., stone corner of secs. $5,6,7$, and 8 , crossroads . ..... T. 13 N., R. 7 E., quarter corner between secs. 6 and 7, center of east and west road, in line with $T$ road north.

Okfuskee-Creek county line, stone marked for old oklahomaIndian Territory boundary line, on north side of angling road, in line with road east on line between secs. 6 and $7, T, 13$ N., R. 7 E.

\begin{tabular}{|c|c|c|}
\hline 353728.5 & 963148.1 & \\
\hline $\begin{array}{lll}35 & 37 & 28.5 \\
35 & 37 & 28.6 \\
35 & 37 & 29.1\end{array}$ & $\begin{array}{lll}96 & 32 & 20.2 \\
96 & 32 & \mathbf{5 2 . 1} \\
96 & 33 & 55.9\end{array}$ & $\begin{array}{l}2,652 \\
2,637 \\
5,268\end{array}$ \\
\hline $\begin{array}{lll}35 & 37 & 28.9 \\
35 & 37 & 28.6\end{array}$ & $\begin{array}{lll}96 & 34 & 28.3 \\
96 & 35 & 00.2\end{array}$ & $\begin{array}{l}2,673 \\
2,635\end{array}$ \\
\hline $\begin{array}{lll}35 & 37 & 28.5 \\
35 & 37 & 28.2\end{array}$ & $\begin{array}{lll}96 & 35 & 32.2 \\
96 & 36 & 04.2\end{array}$ & $\begin{array}{l}2,644 \\
2,642\end{array}$ \\
\hline 353727.0 & 963636.1 & 2,638 \\
\hline 353727.9 & 963715.0 & 3,218 \\
\hline
\end{tabular}

Magnetic declination north and south through center of quadrangle, $10^{\circ} 06^{\prime} \mathrm{E}$; for south border of quadrangle, $9^{\circ} 52^{\prime} \mathrm{E}$.; for east border of quadrangle, $10^{\circ} 44^{\prime} \mathrm{E}$.; east and west through center of quadrangle, $10^{\circ} 11^{\prime} \mathrm{E}$. 
SKEDEE QUADRANGLE.

South along highways near west border of quadrangle. (By B. H. Yoakum in 1916.)

\begin{tabular}{|c|c|c|c|}
\hline Station. & Latitude. & Longitude. & $\begin{array}{l}\text { Distance } \\
\text { between } \\
\text { stations. }\end{array}$ \\
\hline dilepost 44, opposite, center of Atchison, Topeka \& Santa Fe Ry. & 0111 & 0111 & Feet. \\
\hline $\begin{array}{l}\text { track } \\
\text { Road crossing east-west. } 3 \ldots \ldots \ldots \ldots \ldots \ldots \ldots\end{array}$ & $\begin{array}{lll}36 & 29 & 43.1 \\
36 & 29 & 33.4\end{array}$ & $\begin{array}{lll}96 & 43 & 53.5 \\
96 & 43 & 49.8\end{array}$ & 1,035 \\
\hline 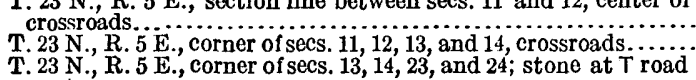 & $\begin{array}{ll}36 & 29.11 .7 \\
36 & 28 \\
41.1\end{array}$ & $\begin{array}{lll}96 & 43 & 41.4 \\
96 & 43 & 41.2\end{array}$ & $\begin{array}{l}2,294 \\
3,091\end{array}$ \\
\hline T. 23 N., R. 5 E., quarter corner between secs. 23 and $24, T$ road & 362749.0 & $\begin{array}{llll}96 & 43 & 41.3\end{array}$ & 5,265 \\
\hline 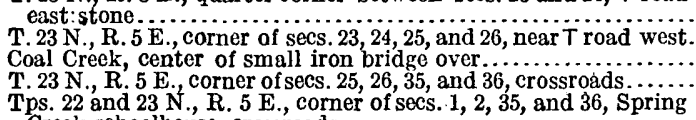 & $\begin{array}{lll}36 & 27 & 23.0 \\
36 & 26 & 56.8 \\
36 & 26 & 44.4 \\
36 & 26 & 04.7\end{array}$ & 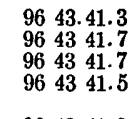 & $\begin{array}{l}2,636 \\
2,643 \\
1,258 \\
4,015\end{array}$ \\
\hline $\begin{array}{l}\text { Creek schoolhouse, crossroads }, \ldots, \ldots \text {, } \\
\text { T. } 22 \text { N., R. } 5 \text { E., corner of secs. } 1,2,11 \text {, and } 12 \text {, center of crossroads. } \\
\text { T. } 22 \text { N., R. } 5 \text { E., corner of secs. } 11,12,13 \text {, and } 14 \text {, in northwest angle }\end{array}$ & $\begin{array}{lll}36 & 25 & 12.6 \\
36 & 24 & 20.2\end{array}$ & $\begin{array}{lll}96 & 43 & 41.2 \\
96 & 43 & 41.2\end{array}$ & $\begin{array}{l}5,265 \\
5,300\end{array}$ \\
\hline $\begin{array}{l}\text { of road forks; iron post stamped "Prim. Trav. Sta. No. } 27,1916 \text { ".. } \\
\text { T. } 22 \text { N., R. } 5 \text { E., corner of secs. } 10,11,14 \text {, and } 15 \text {, center of cross- }\end{array}$ & 362328.3 & $9643 \quad 42.1$ & 5,252 \\
\hline T. 22 N., R. 5 E., corner of secs. $14,15,22$, and 23 , center of cross- & 362328.4 & $9644 \quad 45.7$ & 5,199 \\
\hline T. 22 N., R. 5 E., corner of secs. $22,23,26$, and 27 ; stone at sharp & 362236.1 & 964446.2 & 5,286 \\
\hline T. 22 N., R. 5 E., quarter corner between secs. 26 and 27 , center of & 362144.1 & $9644 \quad 46.1$ & 5,260 \\
\hline T. 22 N., R. 5 E., corner of secs. $26,27,34$, and 35 , in bottom north & $\begin{array}{lll}36 & 21 & 17.8\end{array}$ & $96 \quad 44 \quad 46.8$ & 2,654 \\
\hline 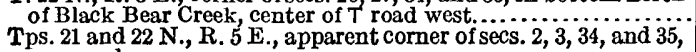 & $\begin{array}{lll}36 & 20 & 51.8\end{array}$ & 964446.1 & 2,630 \\
\hline T. 21 N., R. 5 E., quarter corner between secs. 2 and 3 , on west side & 361959.5 & 964446.3 & 5,295 \\
\hline $\begin{array}{l}\text { of north-south road, at fence line east; stone. } 21 \text { N., R. } 5 \text { F., corner of secs. } 2,3,10 \text {, and } 11 \text {, on north side of } \\
\text { T. }\end{array}$ & $\begin{array}{llll}36 & 19 & 33.2\end{array}$ & $9644 \quad 46.4$ & 2,655 \\
\hline $\begin{array}{l}\text { east-west road, at T road south; stone } 21 \text { N., R. } 5 \text { E., on line between secs. } 10 \text { and } i 1 \text {, near telegraph } \\
\text { pole marked " } 473.20 \text {," St. Louis-San Francisco } R y ., \text { second- }\end{array}$ & 361907.1 & 964446.5 & 2,639 \\
\hline $\begin{array}{l}\text { T. } 21 \text { N., R. } 5 \text { E., corner of secs. } 10,11,14 \text {, and } 15 \text {, crossroads....... } \\
\text { T. } 21 \text { N., R. } 5 \text { E., corner of secs. } 14,15,22 \text {, and } 23 \text {, center of cross- }\end{array}$ & $\begin{array}{lll}36 & 18 & 23.2 \\
36 & 18 & 15.2\end{array}$ & $\begin{array}{lll}96 & 44 & 46.7 \\
96 & 44 & 47.1\end{array}$ & $\begin{array}{r}4,442 \\
811\end{array}$ \\
\hline T. 21 N., R. 5 E., corner of secs. $22,23,26$, and 27 at $\mathrm{T}$ road south, & $\begin{array}{llll}36 & 17 & 23.1\end{array}$ & 964447.0 & 5,263 \\
\hline T. 21 N., R. 5 E., corner of secs. $26,27,34$ and 35 , center of cross- & 361631.1 & 964446.3 & 5,260 \\
\hline 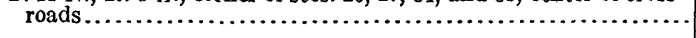 & 361539.5 & 964446.3 & 5,220 \\
\hline
\end{tabular}

Near east border of quadrangle.

\begin{tabular}{l}
$\begin{array}{l}\text { Souter Bend ferry, on north bank of Arkansas River, triangle at } \\
\text { road forks............................................. } 361832.4\end{array} \mid 963016.0$ \\
\hline
\end{tabular}

On road in northeast corner of quadrangle.

\begin{tabular}{|c|c|c|c|}
\hline 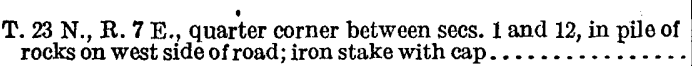 & 362932.6 & 963018.8 & 5,963 \\
\hline
\end{tabular}

Magnetic declination for west border of quadrangle, $9^{\circ} 55^{\prime} \mathrm{E}$. 
SKEDEE QUADRANGLE-Continued.

West along highways through center of quadrangle.

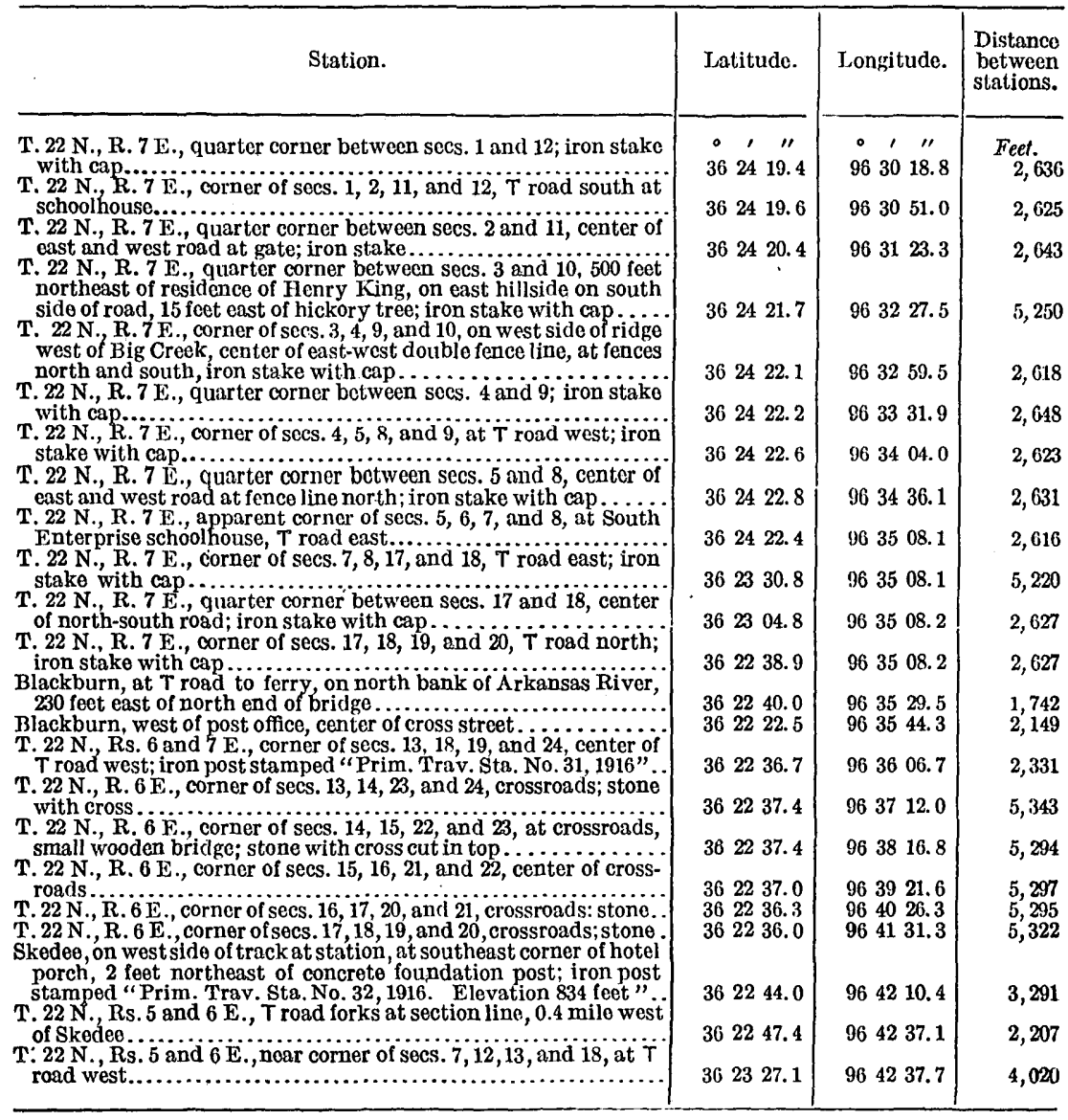

Magnetic declination through center of quadrangle, $9^{\circ} 40^{\prime} \mathrm{E}$.

YALE QUADRANGLE.

East along highways near north border of quadrangle. (By B. F. Yoakum in 1916.)

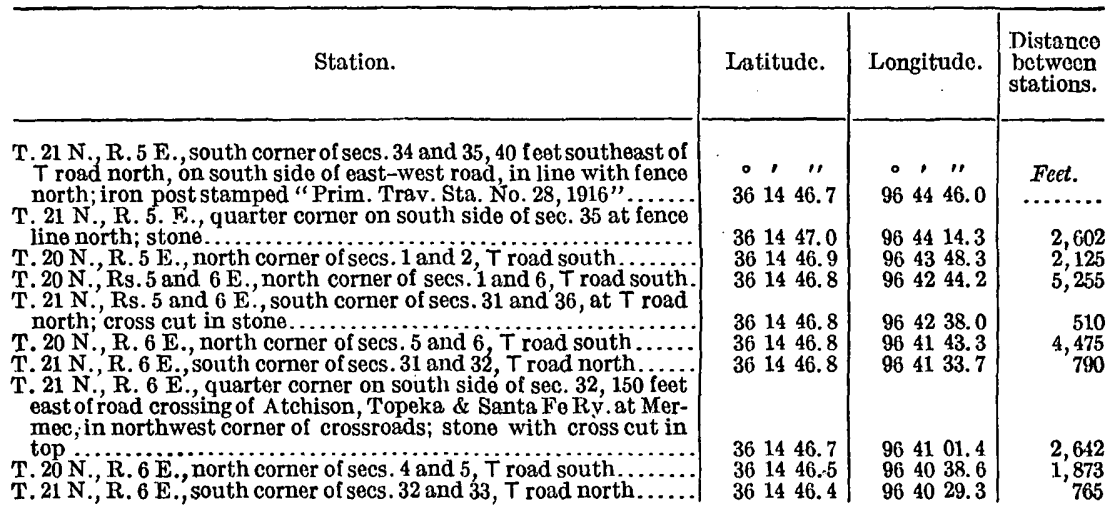


YALE QUADRANGLE-Continued.

East along highways near north border of quadrangle-Continued.

\begin{tabular}{|c|c|c|c|}
\hline Station. & Latitude. & Longitude. & $\begin{array}{l}\text { Distance } \\
\text { between } \\
\text { stations. }\end{array}$ \\
\hline 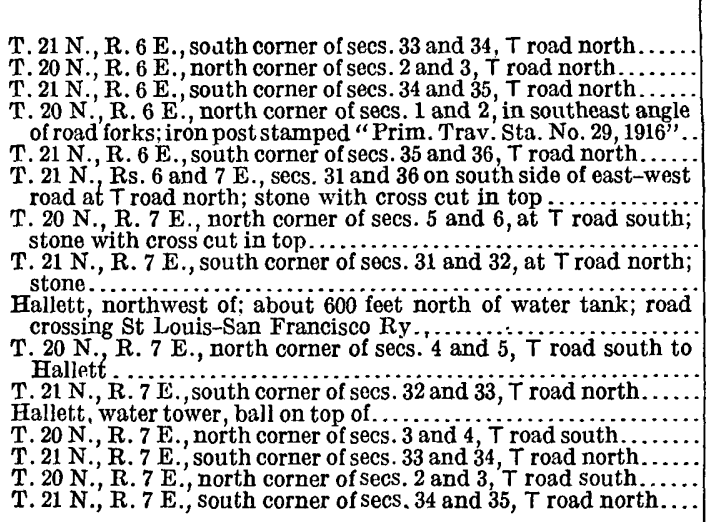 & $\begin{array}{ccc}\circ & \prime & \prime \prime \\
36 & 14 & 46.7 \\
36 & 14 & 46.7 \\
36 & 14 & 46.7 \\
& & \\
36 & 14 & 46.4 \\
36 & 14 & 46.6 \\
& & \\
36 & 14 & 51.1 \\
& & \\
36 & 14 & 46.4 \\
& & \\
36 & 14 & 46.3 \\
& & \\
36 & 14 & 46.4 \\
& & \\
36 & 14 & 46.2 \\
36 & 14 & 46.3 \\
36 & 13 & 43.0 \\
36 & 14 & 46.3 \\
36 & 14 & 46.3 \\
36 & 14 & 46.2 \\
36 & 14 & 46.3\end{array}$ & $\begin{array}{ccc}\circ & \prime \prime \\
96 & 39 & 24.9 \\
96 & 38 & 30.3 \\
96 & 38 & 20.9 \\
& & \\
96 & 37 & 25.5 \\
96 & 37 & 16.4 \\
& & \\
96 & 36 & 12.2 \\
& & \\
96 & 35 & 19.7 \\
& & \\
96 & 35 & 08.1 \\
& & \\
96 & 34 & 49.6 \\
& & \\
96 & 34 & 15.6 \\
96 & 34 & 03.8 \\
96 & 33 & 55.8 \\
96 & 33 & 10.9 \\
96 & 32 & 59.4 \\
96 & 32 & 06.3 \\
96 & 31 & 55.0\end{array}$ & $\begin{array}{r}\text { Feet. } \\
5,270 \\
4,474 \\
770 \\
4,542 \\
740 \\
5,277 \\
4,328 \\
955 \\
1,518 \\
2,785 \\
960 \\
6,434 \\
7,377 \\
941 \\
4,350 \\
932\end{array}$ \\
\hline
\end{tabular}

Magnetic declination for north border of quadrangle, $9^{\circ} 33^{\prime} \mathrm{E}$.

South along highways near west border of quadrangle.

T. 20 N., R. 5 E., corner of secs. $2,3,10$, and 11, crossroads; stone. T. 20 N., R. 5 E., corner of secs. 10, 11, 14, and 15, center of crossT. 20 N., $\dddot{R} .5$ E., corner of secs. $14,15,22$, and 23 , at crossroads; T. 20 N., R. 5 E., corner of secs. $22,23,26$ and 27 , center of crossroads at Lone Elm schoolhouse.

T. 20 N., R. 5 E., corner of secs. $26,27,34$, and 35 , center of east and

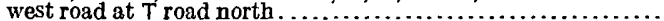

Tps. 19 and 20 N., R. 5 E., corner of secs. $2,3,34$, and 35 , crossroads; stone.

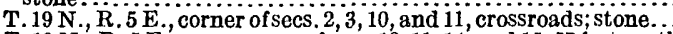

T. 19 N., R. 5 E., near corner of secs. 10, 11, 14, and 15, 57 feet south of $T$ road, in northwest corner of $T$ fence west, 2 feet northwest of corner fence post; iron post stamped "Prim. Trav. Sta. No. 33, 1916 "

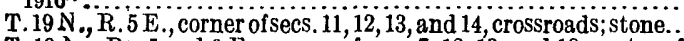
T. 19 N., Rs. 5 and $6 \mathrm{E}$., corner of secs. $7,12,13$, and 18 , center of

T. 19 N., Rs. 5 and 6 E., corner of secs. $13,18,19$, and 24 , crossrosds

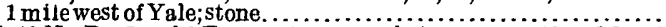
T. 19 N., Rs. 5 and 6 E., quarter corner between secs. 19 and 24 , at

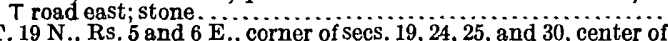
crossroads

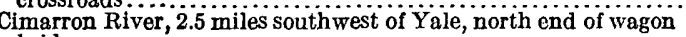
bridg . . . ..................................................... Cimarron River, 2.5 miles southwest of Yale, south end of wagon bridge.

$\mathrm{T} .19 \mathrm{~N} ., \mathrm{Rs} .5$ and $6 \mathrm{E}$., corner of secs. $25,30,31$, and 36 , at cross-

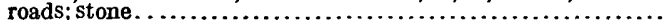

Tps. 18 and 19 N., Rs. 5 and 6 E., corner of secs. $1,6,31$, and 36 , center of crossroads $\ldots \ldots \ldots \ldots \ldots \ldots \ldots \ldots \ldots . \ldots \ldots$ Tps. 18 and 19 N., R. 5 E., corner of secs. $1,2,35$, and 36 , at T road

T. 18 N., R. R E. corner of secs. $1,2,11$, and 12 , center of crossroads.

T. 18 N., R. 5 E., corner of secs. $11,12,13$, and 14 , center of crossT. $18 \mathrm{~N}$., R. 5 E., corner of secs. $10,11,14$, and 15 , center of crossroads.

T. $18 \mathrm{~N},, \mathrm{R} .5 \mathrm{E}$, corner of secs. $14,15,22$, and 23 , at W wgoza school-

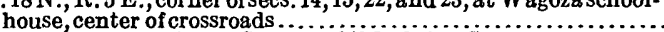

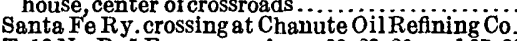

T. 18 N.,R. 5 E., corner of secs. $22,23,26$, and 27,22 feet north by 5 feet west of crossroads, 350 feet south of Mo. Kans. \& Texas Ry. crossing at Ganosiding, 27 miles northeast of Cushing; iron post stamped "Prim. Trav. Sta. No. 34, 1916. Elevation 826 feet Okla"

T. 18 N., R. 5 E., corner of secs. $26,27,34$, and 35 , crossroads; iron

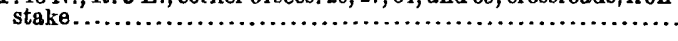

\begin{tabular}{|c|c|c|}
\hline $\begin{array}{lll}36 & 13 & 54.5\end{array}$ & 964452.0 & 5,306 \\
\hline $\begin{array}{lll}36 & 13 & 02.3\end{array}$ & 964452.6 & 5,278 \\
\hline $\begin{array}{lll}36 & 12 & 10.2\end{array}$ & $9644 \quad 53.0$ & 5,261 \\
\hline $\begin{array}{lll}36 & 11 & 17.9\end{array}$ & 964453.3 & 5,291 \\
\hline $\begin{array}{lll}36 & 10 & 25.9\end{array}$ & 964453.6 & 5,261 \\
\hline $\begin{array}{lll}36 & 09 & 33.6 \\
36 & 08 & 41.5\end{array}$ & $\begin{array}{lll}96 & 44 & 54.5 \\
96 & 44 & 55.6\end{array}$ & $\begin{array}{l}5,287 \\
5,274\end{array}$ \\
\hline $\begin{array}{lll}36 & 07 & 48.9 \\
36 & 07 & 49.6\end{array}$ & $\begin{array}{lll}96 & 44 & 57.2 \\
96 & 43 & 53.8\end{array}$ & $\begin{array}{l}5,315 \\
5,200\end{array}$ \\
\hline $\begin{array}{llll}36 & 07 & 49.9\end{array}$ & 964248.3 & 5,378 \\
\hline $\begin{array}{llll}36 & 06 & 57.7\end{array}$ & $\begin{array}{lll}96 & 42 & 49.2\end{array}$ & 5,283 \\
\hline $\begin{array}{llll}36 & 06 & 31.5\end{array}$ & $\begin{array}{lll}96 & 42 & 49.3\end{array}$ & 2,642 \\
\hline $\begin{array}{llll}36 & 06 & 05.5\end{array}$ & $\begin{array}{lll}96 & 42 & 49.8\end{array}$ & 2,635 \\
\hline $3605 \quad 43.4$ & $\begin{array}{lll}96 & 42 & 49.8\end{array}$ & 2,234 \\
\hline $\begin{array}{lll}36 & 05 & 03.3\end{array}$ & $96 \quad 42 \quad 49.4$ & 1,326 \\
\hline $\begin{array}{lll}36 & 05 & 14.3\end{array}$ & $\begin{array}{lll}96 & 42 & 49.7\end{array}$ & 1,612 \\
\hline $\begin{array}{lll}36 & 04 & 21.9\end{array}$ & $9642 \quad 49.9$ & 5,302 \\
\hline $\begin{array}{lll}36 & 04 & 21.7 \\
36 & 03 & 29.3\end{array}$ & $\begin{array}{lll}96 & 43 & 54.1 \\
96 & 43 & 54.3\end{array}$ & $\begin{array}{l}\mathbf{5}, 264 \\
5,294\end{array}$ \\
\hline $\begin{array}{lll}36 & 02 & 37.2\end{array}$ & $9643 \quad 54.5$ & 5,277 \\
\hline $\begin{array}{lll}36 & 02 & 37.3\end{array}$ & $96 \quad 4458.7$ & 5,270 \\
\hline $\begin{array}{lll}36 & 01 & 45.0 \\
36 & 01 & 09.3\end{array}$ & $\begin{array}{lll}96 & 44 & 58.6 \\
96 & 44 & 58.7\end{array}$ & $\begin{array}{l}5,288 \\
3,615\end{array}$ \\
\hline 360053.0 & 964458.8 & 1,645 \\
\hline $3600 \quad 00.5$ & $96 \quad 44 \quad 58.8$ & 5,309 \\
\hline
\end{tabular}


YAIE QUADRANGLE-Continued.

East along highways near south border of quadrangle.

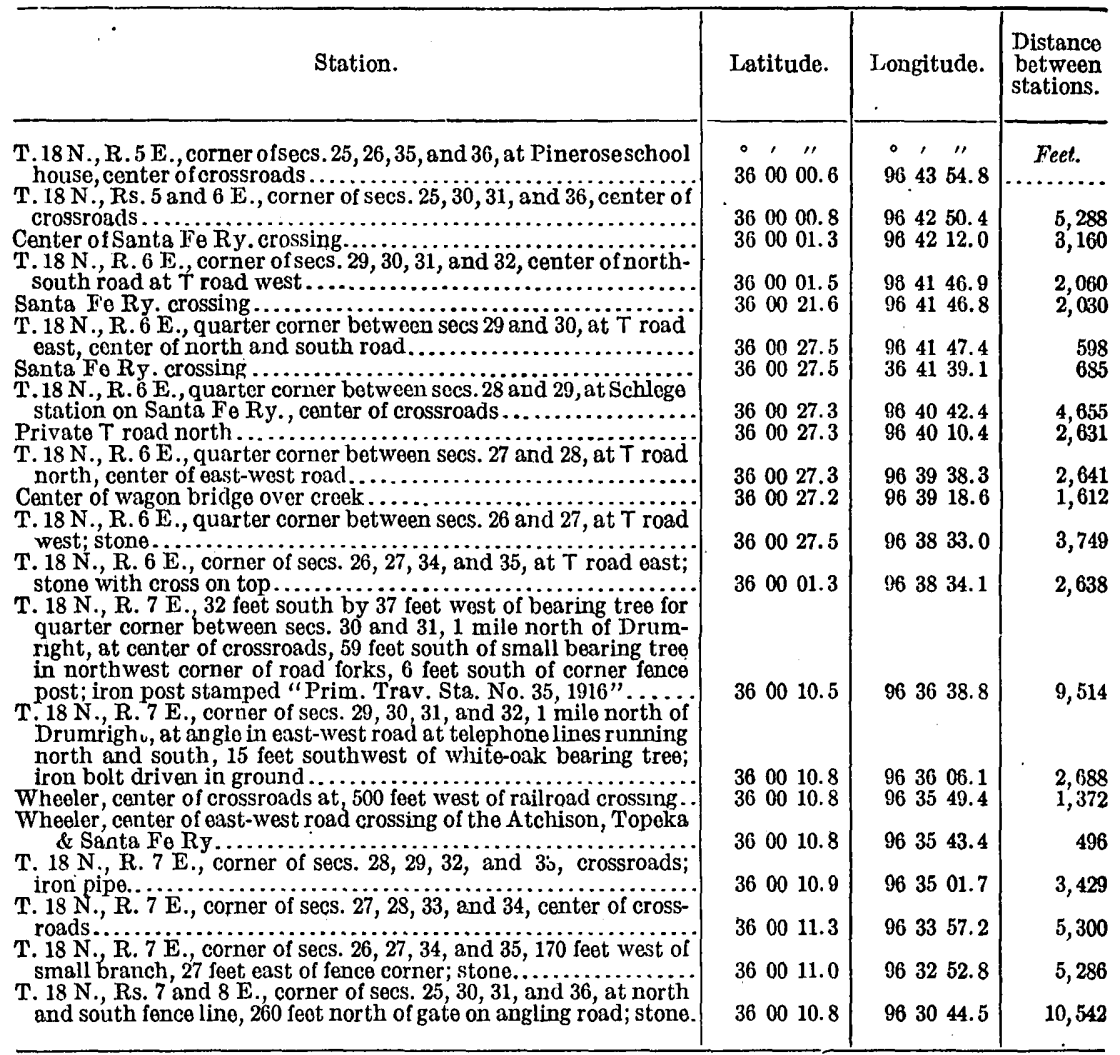


YALE QUADRAT̄GLE-Continued.

West along highways through center of quadrangle.

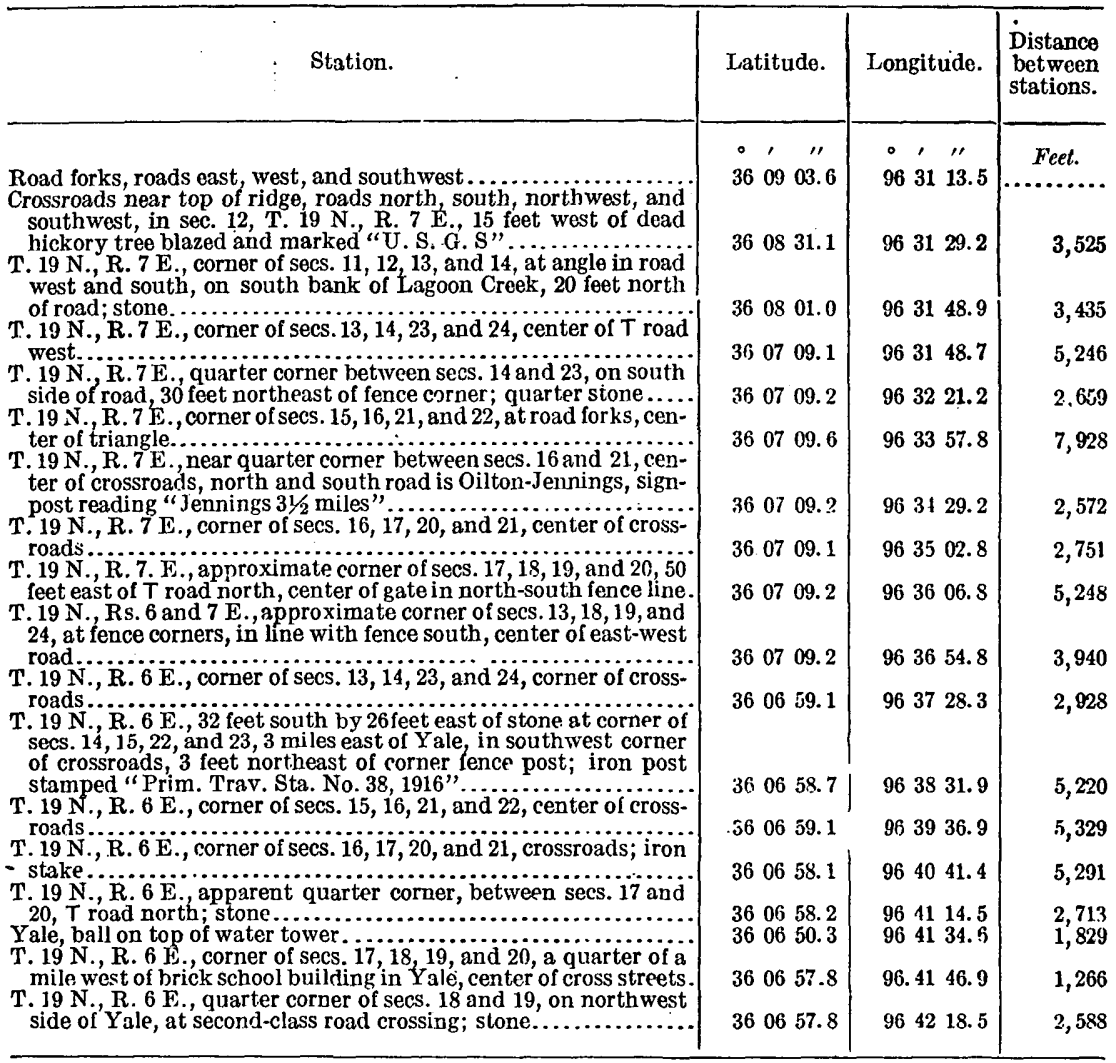

Magnetic declination along west border of quadrangle, $9^{\circ} 01^{\prime} \mathrm{E}$.; along south border of quadrangle, $9^{\circ} 08^{\prime} \mathrm{E}$.; through center of quadrangle, east-west, $9^{\circ} 08^{\prime} \mathrm{E}$. 